Florida International University FIU Digital Commons

10-26-2009

\title{
Relations Between Acculturation And Alcohol Use Among International Students
}

\author{
Ananth Kanaparthi \\ Florida International University, ananth.kanaparthi@gmail.com
}

DOI: $10.25148 /$ etd.FI09120812

Follow this and additional works at: https://digitalcommons.fiu.edu/etd

Part of the Community Psychology Commons, Other Psychology Commons, and the Social Psychology Commons

\section{Recommended Citation}

Kanaparthi, Ananth, "Relations Between Acculturation And Alcohol Use Among International Students" (2009). FIU Electronic Theses and Dissertations. 133.

https://digitalcommons.fiu.edu/etd/133

This work is brought to you for free and open access by the University Graduate School at FIU Digital Commons. It has been accepted for inclusion in FIU Electronic Theses and Dissertations by an authorized administrator of FIU Digital Commons. For more information, please contact dcc@fiu.edu. 


\section{FLORIDA INTERNATIONAL UNIVERSITY}

Miami, Florida

\section{RELATIONS BETWEEN ACCULTURATION AND ALCOHOL USE AMONG INTERNATIONAL STUDENTS}

A dissertation submitted in partial fulfillment of the

requirements for the degree of

\section{DOCTOR OF PHILOSPHY}

in

PSYCHOLOGY

by

Ananth Kanaparthi

2009 


\section{To: Dean Kenneth Furton}

College of Arts and Sciences

This dissertation, written by Ananth Kanaparthi, and entitled Relations between Acculturation and Alcohol Use among International Students, having been approved in respect to style and intellectual content, is referred to you for judgment.

We have read this dissertation and recommend that it be approved.

Marilyn J. Montgomery

Mark Macgowan

Wendy K. Silverman

\section{William Kurtines}

Jonathan G. Tubman, Major Professor

Date of Defense: October 26, 2009

The dissertation of Ananth Kanaparthi is approved.

Dean Kenneth Furton

College of Arts and Sciences

Dean George Walker

University Graduate School

Florida International University, 2009 


\section{DEDICATION}

I dedicate this dissertation to my wife, Raaji. Without her patience,

understanding, continuous encouragement, and love, this work would not have been completed. 


\section{ACKNOWLEDGMENTS}

I express my earnest gratitude to my mentor, Dr. Jonathan Tubman for his belief in my abilities, his uninterrupted encouragement, constructive advice, and his patience through out this endeavor. I remain ever indebted to his time that he has invested during countless meetings, and his exceptionally helpful ideas.

I feel very sanguine to express my gratefulness to Dr. Marilyn Montgomery, who endowed me with and fostered in me a great abilities regarding cross-cultural evaluation, which made my dissertation far better, and effective than it would be otherwise. I appreciate her valuable suggestions. It makes me very happy to extend my gratitude to Dr. Wendy Silverman, for her invaluable suggestions, comments, and in particular, her advice that has reshaped and broadened the scope of my research, which in turn, helped me to learn more. In addition, I sincerely appreciate Dr. William Kurtines for his participation on my committee and his valuable suggestions

during my dissertation proposal defense which gave me better insights regarding my research questions. 


\section{ABSTRACT OF DISSERTATION \\ RELATIONS BETWEEN ACCULTURATION AND ALCOHOL USE AMONG \\ INTERNATIONAL STUDENTS}

by

Ananth Kanaparthi

Florida International University, 2009

Miami, Florida

Jonathan G. Tubman, Major Professor

In the current study, 226 international students attending Florida International University responded to an Web-based questionnaire that assessed self-reported scores for: acculturation, acculturative stress, alcohol use patterns, perceived peer alcohol use, and perceived injunctive social norms for alcohol use. The purpose of the study was to evaluate structural relations among this set of variables using Structural Equation Modeling (SEM) via AMOS 17.0. The results of SEM analyses documented acceptable fit of a model which hypothesized that relations between acculturation and alcohol use variables are mediated partially by acculturative stress, perceived peer alcohol use, and perceived injunctive social norms. While significant path coefficients were documented for paths between (a) acculturation and acculturative stress and (b) acculturative stress and alcohol use, the coefficients had negative signs, contrary to existing studies investigating these relations among immigrant youth. While paths between (a) perceived peer alcohol use or (b) perceived injunctive social norms and participants' alcohol use were significant, path coefficients between acculturation and either (a) perceived peer alcohol use or (b) perceived injunctive social norms were not 
statistically significant. In addition, multiple-group comparisons suggested that social support had a significant moderating influence on several significant paths in the structural model. Specifically, international students reporting higher social support reported lower scores for acculturative stress and alcohol use, in contrast to their counterparts who reported lower scores for social support. 


\section{TABLE OF CONTENTS}

1. $\quad$ STATEMENT OF THE PROBLEM 1

2. LITERATURE REVIEW 12

$\begin{array}{ll}\text { International Students in the United States } & 13\end{array}$

$\begin{array}{ll}\text { Adjustment Issues of International Students } & 14\end{array}$

$\begin{array}{ll}\text { Stress and Coping Models } & 17\end{array}$

$\begin{array}{ll}\text { Academic Stressors } & 21\end{array}$

Demands for English Language Proficiency 23

Other Stressors Studied in Past Research $\quad 24$

$\begin{array}{ll}\text { Coping Mechanisms } & 24\end{array}$

Social Support Models $\quad 27$

Other Models to Explain Adjustment Among International Students 28

Acculturation Framework $\quad 31$

$\begin{array}{ll}\text { Cultural Racism } & 35\end{array}$

Racially Obnoxious Situations $\quad 36$

Collectivism vs. Individualism 36

Comparison of Acculturation and Stress-Coping Processes Models 38

Integrating Concepts of Acculturation and Stress-Coping Frameworks $\quad 40$

Berry's Four Groups Model 41

The Relation of Acculturation Processes to Alcohol Use Patterns 42

More Accepting Social Norms $\quad 43$

Alcohol as a Social Lubricant $\quad 44$

Alcohol Use as an Avoidant Coping Strategy $\quad 44$

Future Research Directions $\quad 48$

$\begin{array}{lr}\text { The Current Study } & 50\end{array}$

$\begin{array}{lr}\text { Hypotheses } & 50\end{array}$

3. METHODS 52

Participants $\quad 52$

$\begin{array}{ll}\text { Measures } & 53\end{array}$

Short Acculturation Scale for Hispanics (SASH) 53

Acculturative Stress Scale for International Students (ASSIS) 55

Alcohol Use Disorders Identification Test (AUDIT) 55

Perceived Peer Norms for Alcohol Use $\quad 57$

Perceived Injunctive Social Norms $\quad 58$

Social Support Questionnaire 6 (SSQ6) 59

$\begin{array}{ll}\text { Other Outcome Measures } & 60\end{array}$

$\begin{array}{lr}\text { Procedure } & 60\end{array}$

$\begin{array}{ll}\text { Analytic Plan } & 61\end{array}$

SEM and AMOS $\quad 63$

Outliers $\quad 64$

Non-Normality $\quad 64$ 
Missing Data $\quad 64$

Model Fit Indices $\quad 65$

4. RESULTS 66

Descriptive Statistics $\quad 66$

Intercorrelations between Acculturation Variables and Alcohol Use

$\begin{array}{ll}\text { Variables } & 69\end{array}$

$\begin{array}{ll}\text { Structural Equation Model (SEM) Analyses } & 72\end{array}$

Model Fit Summary $\quad 72$

Model Fit Indices $\quad 72$

Modification Indices $\quad 74$

Respecification of a Model and its Exploratory Nature $\quad 75$

$\begin{array}{ll}\text { Model Evaluation for the Current Study } & 76\end{array}$

$\begin{array}{ll}\text { Model Summary } & 76\end{array}$

$\begin{array}{ll}\text { Identification of the Model } & 76\end{array}$

Assessment of Normality $\quad 77$

$\begin{array}{ll}\text { Notes for the Model } & 78\end{array}$

$\begin{array}{ll}\text { Modification Indexes } & 79\end{array}$

$\begin{array}{ll}\text { Final Model Statistics } & 83\end{array}$

Standardized and Unstandardized Path Coefficients $\quad 83$

$\begin{array}{lr}\text { Group Differences } & 85\end{array}$

Additional Data Analysis Issues $\quad 89$

$\begin{array}{lr}\text { Missing Data } & 89\end{array}$

$\begin{array}{lr}\text { Excluded Variables } & 89\end{array}$

5. DISCUSSION 91

Integration of the finding of the current study with Past Research 92

Relations between Acculturation and Acculturative Stress 92

Relations between Acculturative Stress and Alcohol Use Outcome 94

Mediating Influence of Peer Modeling and Injunctive Social Norms 95

Moderating Influence of Social Support 98

Gender Differences $\quad 99$

Other Potential Moderating Variables $\quad 100$

Implications for Intervention and Prevention 100

Student Education $\quad 100$

Peer mentors $\quad 102$

$\begin{array}{ll}\text { Limitations } & 103\end{array}$

Reliability of Self-Report Data 103

Inability to Draw Causal Relations and the Issue of A Control Group 104

Cross Cultural Validity 106

$\begin{array}{ll}\text { Future Directions } & 108\end{array}$

$\begin{array}{ll}\text { REFERENCES } & 110\end{array}$

$\begin{array}{ll}\text { VITA } & 145\end{array}$ 
1. Key Demographic Variables Reported by Florida International University International Students

2. Acculturation-Related Behaviors for Florida International University International Student Sample

3. Acculturative Stress Ratings for Florida International University International Student sample

4. Perceived Peer Alcohol Use Behaviors for the Florida International University International Student Sample

5. Alcohol Use Behaviors for the Florida International University International Student Sample

6. Intercorrelations of Acculturation Variables with other Constructs for the Florida International University International Student Sample

7. Intercorrelations of Alcohol Use Behaviors with Other Constructs for the Florida International University International Students Sample

8. Intercorrelations between ASSIS Acculturative Stress Variables and SASH Acculturation Variables for the Florida International University International Students Sample

9. Intercorrelations between Acculturative Stress Variables and Alcohol Use Behaviors for the Florida International University International Student Sample

10. Assessment of Normality from AMOS output

11. Top Ten Observations in Descending Order of Mahalanobis d-squared Values

12. Modification Indices (Regression Weights) from AMOS Output for the Initially hypothesized Model

13. Modification Indices (error Covariances) from AMOS Output for the Initially Hypothesized Model

14. Model Fit Indices from AMOS Output, Initial Model 
16. 95\% Confidence Intervals for Unstandardized Path Coefficients for Final Model

17. Standardized Path Coefficients for Final Model

18. Model Fit Indices Across Models from AMOS Output

19:.Group Differences Across Social Support Groups, Chi-Square Values along with Degrees of Freedom

20. Group Differences across Social Support Groups, Chi-Square Difference Values along with Degrees of Freedom

21. Group Differences across Social Support, Measurement Weights Difference Values

22. Group Differences across Social Support, Structural Weights Difference Values 


\section{CHAPTER 1: STATEMENT OF THE PROBLEM}

The current study is intended to examine relations between acculturation-related processes and alcohol use behaviors among international students attending a university in the United States. There are three major reasons to examine international student populations to document relations between acculturation-related processes and patterns of alcohol use. First, while several past research studies have examined adjustment issues among international students, they were either: (a) based solely on stress-coping frameworks proposed by Lazarus (1985) and others; or (b) broadly atheoretical, without significant integration of acculturation-related constructs. Several studies reported that international students experienced the process of acculturation while making adjustments to educational settings in a host country and they referred to an acculturation framework to examine adjustment issues among international students (e.g., see Sandhu \& Asrabadi, 1994). While several studies have used an acculturation framework to conceptualize the nature of changes experienced by international students, samples have been confined largely to Asian international students, and thus, the generalizability of those findings is limited.

Second, past research on acculturation has documented significant relations between acculturation processes and alcohol use behaviors in acculturating nonuniversity immigrant populations, including both adolescents and adults. The generalizability of research findings generated from mainstream immigrant populations to samples of international students is questionable as international students are likely to differ significantly from mainstream immigrant populations in terms of the diversity of each of these groups, as well as the unique types of peer and family supports they receive 
during acculturation to the host society (Misra \& Castillo, 2004; Sue, 1999). In addition, international students are more likely to experience high demands for language proficiency (Poyrazli, Arbona, Bullington, \& Pisecco, 2001), and significant stressors related to academic demands.

Third, international students represent and experience developmental transitions typical of either late adolescent or young adult age groups, ones more prone to participate in risk behaviors such as alcohol use, compared to younger groups of acculturating adolescents or older groups of immigrants. In addition, international students are more likely to participate in health risk behaviors when in the United States than are comparable groups of youth in their countries of origin. In the United States, the availability of alcohol and other substances is significantly more prevalent, compared to the countries of origin of many international students (Gil, Wagner, \& Vega, 2000). This differential availability may make international students more susceptible to delayed onset of alcohol abuse during transitions within educational settings in the United States. International Students in the United States

International students are enrolled in large numbers at academic institutions in the United States at a range of educational levels including the bachelor's, master's, and doctoral levels. The United States hosts the largest numbers of international students in the entire world (Misra, Crist, \& Burant, 2003) and these numbers are predicted to continue to increase in the future (Hayes \& Lin, 1994; Sandhu \& Asrabadi, 1994). In addition, to add support, a recent study by Misra and Castillo (2004) reported growing enrollment of international students in the recent past. The increasing numbers of international students provides a rationale for increased research that addresses their 
specific needs and developmental processes within educational settings in the United States (Davis, 2002).

\section{Adjustment Issues of International Students}

During transitions in the post-secondary educational system of the United States, international students are likely to experience a range of stressors relevant to adjustment outcomes. Past research studies have identified several factors that influence and regulate adjustment processes among international students in the United States. Several previous studies that examined adjustment issues in samples of international students have used the stress-coping conceptual framework proposed by Lazarus (1985) or others to describe the influence of a range of stressors on adjustment, as well as relevant negative health risk behavior outcomes. The range of stressors studied included, but was not limited to, contextual demands for English language proficiency (Yeh \& Inose, 2003), pedagogical issues involving the host educational institution (Maxwell, Adam, Pooran, \& Scott, 2000), financial stress factors (Yang \& Clum, 1994), and perceptions of racial discrimination (Klonoff, Landrine, \& Ullman,1999; Sandhu, 1994). These studies examined negative mental health adjustment outcomes such as depression, anxiety, and health risk behaviors (Krammer, Prüfer-Kramer, Stock, \& Tshiananga, 2004), academic failure (Michailidis, 1996), suicidal ideation (Cho, 2003), in addition to stressors related to the weather and climate of the host country.

\section{English Language Proficiency}

English language proficiency has been identified by past researchers as a significant predictor of psychosocial adjustment problems among international students. English is the medium of instruction in educational institutions in the United States. 
Therefore, there are significant requirements for international students for minimal proficiency in the spoken, as well as the written use of the English language. Perceptions of stress related to contextual demands for English language proficiency have been reported by past researchers to be a significant influence on the adjustment of international students. International students who were more proficient at English language usage reported better adjustment outcomes and experienced less stress during transitions to new educational settings, compared to international students who were less proficient at the use of spoken and written English (Parr, Bradley, \& Bingi, 1992;

Sadrossadat, 1995; Sandhu \& Asrabadi, 1994; Yeh \& Inose, 2003). In addition, Lin and Yi (1997) found that English language proficiency not only influenced students' academic adjustment but also international students' development of meaningful interpersonal relationships.

\section{Pedagogical Issues and Academic Stressors}

In general, transitions from one educational setting to another are stressful because they require multiple, co-occurring adjustments from students. Comparable transitions between educational institutions are made more challenging for international students since the transitions they experience often involve additional environmental demands including: changes in the location of the educational institution, increases in demands for language proficiency, changes in availability of specific family and social support systems, and added financial burdens. Specific features of the educational system of the United States have also been reported by researchers to influence significantly international students' adjustment (Maxwell, Adam, Pooran, \& Scott, 2000). In contrast to the commonly passive content-based teaching styles of several foreign pedagogical 
systems (e.g., the Southeast Asian system), the more active teaching styles of Western education systems demand more student participation in the classroom. International students have been described as being burdened during this transition due to United States teachers' higher demands for students' classroom participation. Maxwell et al. (2000) also examined others' expectations and perceptions of international students transitioning into host country educational systems, including mentors and other staff, and they reported significant differences between educators' expectations of international students and their perceived ability and performance.

\section{Coping Mechanisms}

While several published studies that examined adjustment outcomes among international students have used conceptual models based upon stress-coping processes proposed by Lazarus (1985) and others, not many of them have examined specific coping mechanisms adopted by international students. In addition, few studies that have examined coping mechanisms employed by international student samples have attempted to identify resources available to international students and their ability and propensity to make use of available social resources. However, the availability of counseling facilities at host universities, informal sources of social support, and financial resources all have been identified as significant resources for international students to facilitate their ability to cope with stressors during the process of adjusting to new educational settings (Constantine, Okazaki, \& Utsey 2004; Maxwell et al., 2000; Misra et al., 2003; Mori, 2000; Randall, Naka, Yamamoto, Nakamoto, Arakaki, \& Ogura, 1988). Utilization of these resources to develop problem-oriented coping mechanisms was found to be related to better adjustment outcomes. In contrast, the inability to access specific social resources 
or the tendency to not make use of available resources was found to result in negative health risk behavior outcomes. For example, attitudes among some Asian international students were related to not accessing available counseling resources and associated with negative mental health outcomes, such as depression and anxiety (Nilsson, Berkel, Flores, \& Lucas, 2004; Yi, Giseala, \& Kishimoto, 2003).

\section{Family and Social Support Factors}

International students who come from countries that foster collectivistic cultural norms tend to depend on social support, family support and support from significant others when engaged in coping with stress (Constantine et al., 2004; Maxwell et al., 2000; Misra et al., 2003; Mori, 2000; Randall et al., 1988). In contrast, Western cultures, and in particular the culture of the United States, foster individualistic social norms and emphasize not dependence on others, but independence and self-sufficiency. Lack of fit between these sets of cultural norms may result in situations where international students perceive that they do not receive as much social support from their host country student friends, mentors, and co-workers as they might expect to receive (Mallinckrodt \& Leong, 1992; Misra et al., 2003). Reduced levels of social support or less than adequate social resources for coping may represent significant burdens for international students while creating barriers to their attempts to develop problem-oriented coping strategies. One result of lack of adequate social resources for coping with multiple environmental demands may be the adoption of avoidance coping strategies and participation in negative health risk behaviors, including maladaptive patterns of alcohol or other drug (AOD) use. 


\section{Acculturation among International Students}

While several published studies identified a range of salient stressors for international students, few studies have examined acculturation processes that were relevant to adjustment among international students in a host country such as the United States. The published studies that have addressed acculturation-related processes have identified factors such as cultural identity, cultural gaps between native cultures of the students and the host United States culture, and perceptions of racism or racial discrimination as significant influences on adjustment processes among international students (e.g., Klonoff, Landrine, \& Ullman,1999; Sandhu \& Asrabadi, 1994; Unger, Trinidad, Weiss, \& Rohrbach, 2004). Hence, these researchers found a conceptual framework integrating acculturative processes to be useful for understanding adjustment processes and related outcomes among international students. These studies may represent an initial extension of acculturation research conducted on immigrant samples to international student samples. While these studies have used an acculturation framework to describe adjustment processes among young adult international students, the samples recruited generally have been restricted to Asian international students, and thus the generalizability of these studies are limited to that populations of international students. The current study attempts to address this significant gap in the literature by applying an acculturation framework to the description of adjustment processes and health risk behaviors (e.g., problem alcohol use) among international students attending an urban university in the United States. 


\section{Generalizability of Mainstream Acculturation Research to International Students}

Many published research studies that have examined relations between acculturation processes and adjustment outcomes in mainstream immigrant populations have documented significant relations between acculturation-related processes and negative adjustment outcomes, and in particular, alcohol use behaviors. Past research has identified three different factors that may account for the relation between acculturation processes and alcohol use: (i) social norms that condone alcohol use in the United States; (ii) the use of alcohol to facilitate or enhance social experiences during acculturation by immigrants; and, (iii) the use of alcohol as an avoidance strategy to cope with acculturative stress. While these generic models of the experiences of mainstream immigrant groups may appear to be extendable to any immigrant population, specific variables may restrict the generalizability of mainstream acculturation research to international student populations. The generalizability of the findings from studies of mainstream immigrant populations to international student populations may be limited. International students differ from mainstream immigrant populations in many respects, including the demands they experience for higher levels of English language proficiency, demands experienced during adjustment, unique types of family supports they receive, and added academic stressors encountered during their adjustment experiences, in addition to acculturation-related stressors.

\section{Diversity of International Students}

International students are a diverse population in terms of age, racial/ethnic identity, cultural distance (i.e., the gap between the native culture of the student and American host culture) and several other variables. While studies of mainstream 
immigrant populations generally include one or more racial/ethnic groups, that constitute relatively homogenous groups, research findings may be questionable with regard to their generalizability to international student populations. In addition, studies using different populations (in terms of racial/ethnic identity, age group, or gender) found differing magnitudes of correlations between acculturation-related stressors and specific negative health risk behaviors, and in particular, alcohol use (Chen, Unger, Cruz, \& Johnson, 1999; Vega, Gil, \& Zimmerman, 1993). While variations in research findings may be the result of conceptual and methodological issues, factors such as the characteristics of study populations related to age, gender, and ethnicity might also have contributed significantly to differences in research results. Thus, it is important to examine if international students who constitute a significantly different population also manifest differences in magnitude of relations between acculturation processes and alcohol use. In addition, examining relations between acculturation-related processes and alcohol use behaviors among international students is important for other reasons including: documenting the specific needs and adjustment processes of increasing numbers of international students in the U.S., using this research to reduce the stressfulness of international students' transitions into the United States; preventing international students from developing problem health behaviors, and identifying protective factors present among international students to enhance prevention programs addressing health risk behaviors on campuses.

Last, Gil, Wagner, and Vega (2000) reported that alcohol and other illicit substances are more widely available in the United States, compared to the native countries of most immigrant youth. Several studies have reported that adolescence and 
young adulthood are periods of peak risk for the onset of use and the abuse of alcohol and other illicit drugs. International students are either adolescents or young adults who are susceptible to the onset and escalation of health risk behaviors including Alcohol and Other Drug (AOD) use. Therefore, greater availability of alcohol and other drugs in the United States increases their vulnerability to the development of AOD use problems.

\section{The Current Study}

As indicated by the existing research literature, it is important to examine relations between acculturation processes and alcohol use behaviors among international students for several reasons including: the limited generalizability of research on mainstream immigrant populations, the lack of sufficient research on international student samples using acculturation frameworks, the developmental issues encountered by international students which make them susceptible to health risk behaviors, and the greater availability of alcohol and illicit drugs in the United States compared to their native countries. Hence, the current study is designed to address gaps in the existing research literature by documenting acculturation-related processes, alcohol use behaviors, and relations between these two constructs in a sample of international students.

Four specific hypotheses were evaluated including:

1. Higher scores for indices of acculturation predict significantly higher scores for alcohol use among international students.

2. Acculturative stress partially mediates the relation between acculturation levels and alcohol use among international students.

3. Perceived peer modeling of alcohol use partially mediates the relation between acculturation levels and alcohol use among international students. 
4. Perceived social norms of the host university partially mediate the relation between acculturation levels and alcohol use among international students. A secondary goal of the study included examination of the potential moderating influence of social support on hypothesized relations in the structural model evaluated (i.e., between the levels of acculturation, putative mediators, and alcohol use. It was hypothesized that among international students reporting low levels of social support, (a) acculturation levels would be related more strongly to acculturative stress, and (b) that acculturative stress would be related more strongly to alcohol use. 


\section{CHAPTER 2: LITERATURE REVIEW}

There are three significant reasons to study international student populations to document relations between acculturation-related processes and alcohol use. First, past research has found significant relations between acculturation-related processes and alcohol use in mainstream immigrant populations including a range of ethnic/racial groups in America. However, the generalizability of these findings to international student populations is questionable for several reasons, including the diversity of international students, the unique types of peer and family support they receive during acculturation to the host society (Misra \& Castillo, 2004; Sue, 1999), high demands for language proficiency (Poyrazli, Arbona, Bullington, \& Pisecco, 2001), and additional significant academic stressors. Second, most past research that has examined international students' adjustment has relied either on stress-coping models, social support models, or was broadly atheoretical and has used inadequate research designs. Third, international students typically represent either late adolescent or young adult age groups that are vulnerable to engaging in health risk behaviors, including alcohol use. In the United States, the availability of alcohol is greater, compared to the home countries of many international students (Gil, Wagner, \& Vega, 2000). This greater availability makes international students more susceptible to the onset of alcohol use and abuse.

The goals of this literature review are to: (a) emphasize the importance of research that examines the acculturation-related experiences and adjustment of international student populations, (b) critically review past research that has examined international student populations to document their adjustment, (c) review existing acculturation models and discuss the advantages of using them to understand better 
developmental processes among international students, and finally, (d) discuss relations between acculturation-related processes and alcohol use behaviors.

International Students in the United States

International students come to the United States each year to seek opportunities for quality education. The United States hosts more international students than any other country in the world (Misra, Crist, \& Burant, 2003). International students are enrolled in large numbers at academic institutions in the United States. Approximately 547,000 students, who represent as many as 186 nations, attended 2,500 American institutions of higher education in the academic year 2000 (Davis, 2002; Misra et al., 2003) and this number has increased by $6.4 \%$ during the 2002 academic year (Misra et al., 2003). These figures include both colleges and universities. International students comprise $2.7 \%$ of all bachelor's degree students, $11.4 \%$ of graduate students, and $33 \%$ of all doctoral students in the United States. In the 2002 academic year, approximately one-fifth of all the doctoral degrees awarded by United States institutions and one-third of the doctorates in engineering, mathematics, and the physical and biological sciences were earned by international students (Misra et al., 2003).

According to past research, the international student population in the United States is growing steadily (Misra \& Castillo, 2004). Economic, cultural and political factors influence the enrollment of international students (Hayes \& Lin, 1994; Sandhu \& Asrabadi, 1994) and enrollment numbers are anticipated to increase further in the foreseeable future. For example, Ying (2005) found increased education-related immigration along with ongoing processes of economic and industrial globalization. These ongoing demographic shifts call for conceptual and practical changes in how 
psychologists are trained to work with international students (Constantine, Okazaki, \& Utsey, 2004). As the numbers of international students increase, additional research that examines the specific needs of, and developmental processes among, this population will be needed (Constantine et al., 2004; Davis, 2002). However, despite increasing rates of enrollment, research on international students' adjustment problems has not increased proportionately (Misra \& Castillo, 2004; Smart \& Smart, 1995). For example, Misra and Castillo (2004) examined past research and found many studies that examined the impact of stress and stressors on college students, but studies with samples of international students were not proportionately represented. Modified practice and service provision models, such as the provision of culturally sensitive training for psychologists to assist them to work more effectively with international students are needed to help these students to adjust better to their academic settings in America (Constantine et al., 2004). Adjustment Issues of International Students

Psychosocial adjustment is defined as the adaptation of individuals to new social contexts with regard to specific domains of personal and social functioning, resulting from dynamic interactions between the individual and multiple social contexts (Sadrossadat, 1995). Researchers who studied adjustment among college student populations have focused on the adaptation of students from the high school environment to college or university contexts. Past research on college adjustment has included various dimensions of adaptation including: adjustment to new academic settings, collegiate social norms and traditions, as well as increased responsibilities (e.g., financial responsibilities) which add substantial burdens to the academic adjustment issues. Language challenges, adjustment to the American education system and cultural 
differences were additional aspects that were included in research on the adjustment of international students (e.g., Parr, Bradley, \& Bingi, 1992; Sandhu \& Asrabadi, 1994). The ability to adjust is crucial for the success of international students in a host community as they attempt to achieve their academic goals (Sadrossadat, 1995).

International students are exposed to contemporary American culture and they undergo a transition from their traditional native culture to contemporary American culture during this phase and they may experience a great deal of stress (Hechanova-Alampay, Beehr, Christiansen, \& Van Horn, 2002; Sandhu \& Asrabadi, 1994).

As international students come to the United States from many countries located in Africa, Asia, Europe, and South America, they constitute a diverse population in which a range of risk and protective factors may mediate or moderate relations between acculturation-related factors and negative health risk behaviors. These risk or protective factors may either increase or decrease the probability of specific health risk behaviors during this adjustment phase (Constantine et al., 2004; Misra \& Castillo, 2004; Sue, 1999). For example, gender roles defined in many Eastern Asian cultures, prohibit women from drinking alcohol. This cultural practice might work as a protective factor against the development of alcohol use behaviors, which are significant health risk behaviors among young adults in the United States. Important risk/protective factors might also include some characteristics fostered or not fostered within certain cultures, and these qualities may influence and regulate adjustment processes among international students. For example, self-efficacy, the confidence of individuals in their abilities to implement specific behaviors, are not fostered in communal, collectivistic cultures and this might act as a risk factor during adjustment processes for those international students 
who come from collectivistic cultural traditions. Black and Mendenhall (1990) found that adjustment was strongly influenced by levels of self-efficacy. Students with high levels of self-efficacy were found to have less adjustment-related stress. Hence, it is important to identify factors significantly associated with adjustment processes and risk behavior outcomes among international students.

Constantine et al. (2004) have stated that psychologists are showing increasing interest and concern regarding understanding the adjustment-related issues of international students. Studies, such as those conducted by Mori (2000), Pederson (1991), and Winkleman (1994), have tried to describe the intricate processes underlying the adjustment of international students (Constantine et al., 2004). The adjustment of international students in the United States is influenced by many important factors such as pedagogical issues (Maxwell, Adam, Pooran, \& Scott, 2000), social support factors, including family support (Krämer, Prüfer-Kramer, Stock, \& Tshiananga, 2004; Lee, Koeske, \& Sales, 2004), English language proficiency (Misra \& Castillo, 2004; Poyrazli et al., 2001; Yang \& Clum, 1994), as well as weather and climate differences. Many past research studies have used stress and coping or social support models to depict and examine adjustment processes among international students (e.g., Constantine et al., 2004; Krämer et al., 2004; Maxwell et al., 2000; Misra \& Castillo, 2004; Poyrazli et al., 2001; Yang \& Clum, 1994). Typically, these models have tried to examine one or more individual stressors as predictors of levels of adjustment and negative outcomes among international students. A discussion of the conceptual frameworks of stress-coping and social support models, along with empirical examples of relevant published research 
studies that have examined international student populations using these models, are summarized in the following sections.

\section{Stress and Coping Models}

Traditional transactional models of stress define stress as the demand or set of demands made by the environment (both internal and external) that often result in imbalance or lack of equilibrium by disturbing the functioning of the individual in physical and/or psychological domains (Lazarus, 1985; Thoits, 1995). Dynamic interactionist models of developmental psychopathology also assert that when an individual interacts with the social environment, specific demands are made by the environment that might require more resources to meet those demands than the individual is endowed with, resulting in significant burdens on the self system (Cicchetti, 1989; Sameroff \& Fiese, 1990). While more recent studies (e.g., Moos \& Schaefer, 1993; Schwarzer \& Schwarzer, 1996) have proposed various models to explain stress and coping, conceptually most of them have followed the framework proposed by Lazarus, DeLongis, Folkman, and Gruen (1985).

Coping strategies are fundamentally behavioral and/or cognitive efforts to deal with specific stressors (Ben-zur, 2005; Lazarus et al., 1985). Coping is a mechanism of dynamically changing cognitive and behavioral efforts to manage specific external and/or internal demands that are appraised by individuals as burdensome or exceeding the resources of the person (Lazarus et al., 1985). According to Lazarus et al., (1985) coping involves evaluations and re-evaluations of environmental stressors and the personal resources necessary to manage them. When an individual encounters stressors, he/she makes initial cognitive appraisals of those stressors to assess if existing resources are 
adequate to address the stressors or not. This appraisal determines how the individual responds to the stressors. Based on how individuals address specific stressors, coping styles are classified into two fundamental categories, namely, problem-oriented and avoidance coping styles (Compas, Malcarne, \& Fondacaro, 1988). If personal resources are sufficient to cope with the stressors, problem-oriented coping styles are typically adopted, in which the stressors that are responsible for disequilibrium are identified by the individual and strategies are implemented to reduce their influence by acting against them via the utilization of readily available resources. Strategies such as conflict-solving skills, specific problem-solving skills, cognitive and behavioral modifications constitute examples of some key problem-oriented coping strategies (Grey, 2000). Fostering selfefficacy skills, such as self-assertiveness, can help students adopt problem-oriented coping strategies. For example, as language difficulties pose significant problems for international students during acculturation, gaining language proficiency, and enhancing communication skills can function as useful problem-oriented coping strategies.

In contrast, when individuals select and implement avoidance coping styles, they generally attempt to seek strategies by which they can avoid the influence of stressors for brief periods and temporarily escape from them. A range of cognitive and behavioral avoidance coping strategies are adopted by individuals when they perceive that their available resources can not address stressors adequately or efficiently (Spaccarelli, 1994). For example, when faced with stressful experiences of language proficiency demands, adopting a problem-oriented coping style, an individual often attempts to improve his/her language proficiency to counter the influence of the stress experienced. In contrast, if an avoidant coping style is adopted, an individual may attempt to avoid environments in 
which language proficiency is highly required. In general, avoidance coping styles are adopted when an individual cannot access sufficient available resources to adopt problem-oriented coping strategies. Avoidance coping strategies provide the individual with only a temporary solution but the stressor is likely to regain its influence after a brief period (Ben-zur, 2005; Lazarus et al., 1985; Weyers, Ising, Reuter, \& Janke, 2005).

Avoidance coping strategies also include health risk behaviors such as adopting alcohol and other drug (AOD) use and abuse behaviors to escape temporarily from aversive stressors. This strategy might lead to a repetitive cycle of stress in which avoidant coping behaviors, such as alcohol or other drug use lead to additional stress, and subsequent patterns of heavy, maladaptive AOD use eventually promotes the development of serious psychopathology (Lazarus et al., 1985; Spaccarelli, 1994). The quality of adjustment outcomes are determined by the appropriateness of the coping strategies used to meet effectively the demands generated by new or changing environments. Variability in cognitive appraisals and the coping styles used is a significant predictor of mental health outcomes (Ben-Zur, 2005; Li, Seltzer, \& Greenberg, 1999; Spaccarelli, 1994). Applied consistently, avoidance coping strategies often result in negative health outcomes, including depression and anxiety, as stressors experienced are avoided successfully for brief periods, but reappear and act on the individual as soon as the reprieve induced by avoidance coping is over. Specific emotionfocused coping styles include adopting behaviors such as avoidance, distancing, and showing attitudes of finding positive value in negative events (Grey, 2000).

While several models of coping suggest that the selection of coping strategies (whether problem-oriented or emotion-focused) depend on situation-specific availability 
of coping resources (Folkman \& Lazarus, 1991), other models of coping suggest that consistent patterns of selections of specific coping strategies are determined in part by specific traits of the individual, independent of situation-specific factors (Weyers et al., 2005). However, effective problem-oriented coping styles eliminate or reduce the magnitude of the problem and reduce the influence of stress. Hence, reduced distress and reduced psychological arousal result as a consequence of the adoption of problemoriented coping strategies. Emotion-focused or avoidance coping styles have been found to be correlated significantly with negative health outcomes, such as psychological distress, negative affect, distress (Ben-Zur, 2005), and depression (Li, Seltzer, \& Greenberg, 1999).

Several previous studies conducted with samples of international students have used the stress-coping conceptual framework to describe the influence of a range of stressors on students' adjustment, including negative health risk behavior outcomes. For example, Yeh and Inose (2003) studied the influence of stress arising from English language ability demands. Maxwell et al. (2000) studied stress induced by pedagogical issues to understand significant adjustment issues among samples of international students. In addition, previous studies (e.g., Klonoff, Landrine, \& U1lman, 1999; Sandhu, 1994) also found that stress associated with perceptions of racial discrimination negatively influenced and intensified the stressful experiences of international students.

While Misra et al. (2003) did not find a strong influence of financial stressors on adjustment outcomes among international students, studies such as that by Yang and Clum (1994) emphasized the potential influence of financial stress factors on international students' adjustment. These studies examined negative mental health 
outcomes such as depression, anxiety, as well as academic failure. Michailidis (1996) found that stress reported by international students contributed to their lagging behind in terms of academic achievement, in contrast to their host group counterparts. Cho (2003) in a study of international students' adjustment, found that suicidal ideation along with depression resulted from stress during the transition to a host university. Kramer et al. (2004) examined an international student sample from a German university and found that international students experienced more stress, and were more prone to health risk behaviors, compared to native-born students. They also found that stress among international students was associated significantly with mental health symptoms such as anxiety and depression.

Most of the past research that has examined international student samples to identify potential causal relations between stress factors and health risk behavior outcomes have concentrated on academic and pedagogical stressors and stressors related to English language proficiency demands. A further detailed review of those research domains is described in the following sections to capture the current state of affairs in the area of adjustment-related research on international students.

Academic Stressors

The transition from high school settings to collegiate settings may involve stressful adaptation processes for many students, including both native-born and foreign students. This transition will be more stressful for those who come from other countries, as they experience other environmental demands while undergoing normative academic transitions. International students were found to report significant difficulties adapting to unfamiliar educational systems found in the United States (Misra \& Castillo, 2004). 
According to Essandoh (1995), Misra and Castillo (2004), and Mori (2000), the added stressors of learning a new culture, and acquiring new language skills compound normative academic stressors, aggravate students' stress and impose further burdens on them.

Maxwell et al. (2000) examined pedagogical frameworks and the learning adjustment problems of international students of Southeast Asian origin in a Western European university. They looked at the educational experiences of those students, their expectations, with a comparative analysis of the perceptions and interpretations of their behaviors by their mentors and staff. Maxwell et al. (2000) made thorough comparisons between the content-based passive teaching styles of South East Asian cultures and the vocational, active teaching styles of Western cultures. Maxwell et al. (2000) found that there were marked differences between the expectations and realities that both teachers and students held for each other, which is likely to influence negatively the students' academic performance. Maxwell et al. (2000) examined several issues that influence the adjustment of international students such as, differences in education systems, language barriers, as well as the expectations of faculty and students for each other. While the study did not explicitly label any of these issues as stressors, the broader conceptual framework used was similar to the stress-coping framework, and the stress resulting from these stressors, and the influence of these stressors on adjustment were examined in the study.

Many international students, who come to the United States for higher education opportunities, are top-level students in their country of origin. Difficulties experienced during the process of adapting to a new educational system might result in their failure to 
gain similar top positions in competitive academic settings in America, the failure to meet their own and their teachers' expectations, and negative adjustment outcomes such as depression and despair (Misra et al., 2003; Mori, 2000; Randall et al., 1988). The contextual demands of educational settings impose burdens on the students which could lead to the generation of additional stress (Misra et al., 2003; Mori, 2000; Randall et al., 1988).

Demands for English Language Proficiency

Language proficiency is essential for international students as this is the medium of instruction, and learning is facilitated only when one is minimally proficient at using the English language. Poyrazli et al. (2001) examined specific adjustment issues of Turkish students studying in one university in United States and found that international students who are more proficient at the English language adjusted more easily to new educational environments in the host country. Low or inadequate levels of language skills hampered students not only from optimal academic achievement (Lin \& Yi, 1997), but also from developing new interpersonal relationships, seeking social support, and from leading a more hassle-free daily life (Nwadiora \& McAdoo, 1996). Compared to variables such as age, gender, education level, or marital status, English language proficiency has been found to be a more potent predictor of international students' adjustment outcomes (Misra et al., 2003; Yang \& Clum, 1994). In a study on refugee immigrant populations, Nicassio (1983) found that lack of English language proficiency, and Socio Economic Status (SES) were significantly correlated with feelings of alienation from American culture. This lack of proficiency in English language skills 
may inhibit a student from accessing fully the host culture and educational opportunities in it.

\section{Other Stressors Studied in Past Research}

Stereotyping of international students. The assumption that international students are a homogenous population may lead to stereotyping (Misra et al., 2003). Perceptions that they are stereotyped and considered to be socially marginal results in frustration among many international students (Misra et al., 2003). Rather than trying to understand the needs of international students, mentors and other students often treat international students as having problems with adjustment and as needing help. International students often have their own culturally-influenced processes of learning, and academic staff may misinterpret this and label international students as the students with special needs (Maxwell et al., 2000). This labeling of international students conveys a negative attribute and may contribute to a sense of discrimination and social isolation. This perception of discrimination often results in students' reduced ability to learn new skills, increasing their anxiety regarding academic pursuits (Maxwell et al., 2000).

\section{Coping Mechanisms}

A range of cognitive and behavioral strategies that are adopted to cope with stress might be classified as either problem-oriented or avoidance coping styles. The Transactional Model introduced by Lazarus (1985) discussed at length how stressors affect individuals and how social and personal resources help individuals to cope with stressors. Use of personal resources includes learning problem-oriented cognitive and behavioral coping skills and improving existing skills to meet contextual demands. Social 
resources might include seeking help, advice, moral and physical support from one's social network of friends or relatives.

Research on the adjustment of international student populations has examined the availability and utilization of specific personal and social resources that are necessary to cope with stressors related to school and cultural transitions. These studies included assessment of personal and social resources such as seeking counseling (e.g., Nilsson, Berkel, Flores, \& Lucas, 2004; Yi, Giseala, \& Kishimoto, 2003), financial help (e.g., Mallinckrodt \& Leong, 1992) and the use of other social resources (e.g., Mallinckrodt \& Leong, 1992; Nilsson et al., 2004). Several problem-oriented coping strategies, such as seeking counseling services provided in school to cope with cultural stressors, seeking financial aid to cope with the financial stressors, as well as seeking social resources, such as help from social networks of co-ethnic groups or host group members have been examined in past research studies. Many of these studies have identified social support as a particularly important resource that buffers students from stressors, but the results of these studies also documented that international students were often lacking adequate levels of social support.

Collegiate stressors such as scholarship requirements, financial burdens, academic competition-related stressors, and pressures to get good grades are experienced by both international and native-born American students (Cheng, Leong, \& Geist, 1993). However, these two groups of students may differ significantly in the ways in which they perceive stressors, as well as the ways they cope with specific stressors. Coping strategies are developed by individuals, based on their cultural values, as well as the principles of the society in which they were brought up. For example, international students who come 
from collectivistic culture-based countries are more likely to depend on social support, such as support from significant others (Constantine et al., 2004; Maxwell et al., 2000; Mori, 2000; Misra et al., 2003; Randall et al., 1988). As they often lack adequate levels of social support in a new cultural setting, they may become more vulnerable to stress, compared to their American student counterparts. They may lag behind significantly in academic settings because of inappropriate or ineffective coping strategies. They may fail to compete competently with their American counterparts and eventually may result in failure to meet their own academic expectations and goals, further aggravating their perceived stress. Ineffective emotion-focused coping styles such as minimizing, distancing, and avoiding specific situations which might not demand effective behavioral or cognitive efforts also may fail to reduce the impact of stressors in the long term. Adoption of negative health risk behaviors such as alcohol or substance use as coping strategies might further exacerbate the impact of contextual stressors.

While past research studies on international students have attempted to explain their adjustment by examining the stressors they experienced, not many of these studies have tried to examine the coping styles adopted by international students. This is another dimension where research on international students is largely incomplete and fragmented. Few of the available studies have focused on the resources available to international students that might help them to develop problem-oriented coping strategies (Constantine et al., 2004; Maxwell et al., 2000; Mallinckrodt \& Leong, 1992; Yi, Giseala, \& Kishimoto, 2003). The available studies have critically evaluated the range of available resources, as well as the extent to which international students utilize them. International students experience the influence of a wide range of stressors. Past researchers have 
found that international students either underutilize available resources or they do not utilize them at all while coping with those stressors (Misra et al., 2003; Mori, 2000; Nilsson, Berkel, Flores, \& Lucas, 2004; Randall et al., 1988). The most prevalent coping strategies adopted by international students include seeking language enhancement training, seeking faculty help, or seeking financial help from the college or university to make their adjustment process easier (Mallinckrodt \& Leong, 1992; Misra et al., 2003; Randall et al., 1988). Although they adopt various coping strategies to withstand the influence of specific stressors, the majority of international students incur negative outcomes due to either insufficient personal and social resources, or the adoption of avoidance coping mechanisms.

\section{Social Support Models}

Social support is hypothesized to moderate stress symptoms, but international students in general do not receive sufficient social support when they experience stress (Kramer et al., 2004; Lee et al., 2004). Studies by Pederson (1991) and Misra and Castello (2004) found that international students were often deprived of their traditional sources of assistance for coping with stress, such as familial support and other normative sources of social support. Most non-Western cultures foster collectivistic social norms and interdependence among members of society in the face of stressors common to cultural group members. For them, these sources of social support serve as powerful buffers against stress and help them to cope with perceived stress (Mallinckrodt \& Leong, 1992; Misra et al., 2003). Through communication, ventilation of stress, taking advice, moral support, or financial help, individuals seek help from their own social networks, including friends and family members. 
In contrast to individuals brought up in collectivistic cultures, individuals brought up in individualistic cultures show different attitudes in response to the support seeking cues of other members of the social group. Being brought up in an individualistic culture, host group members, in general, rarely feel the need to extend support to international students when they need this resource and this lack of sensitivity towards the needs of international students may lead to their perceptions of being hated, or being discriminated against. Thus, lack of readily extended social support by host group members may further worsen the existing stress felt by many international students. This might be one significant reason for the failure of international students to develop deep cross-cultural relationships with host group members or to be able to rely on them for social support. Bulthuis (1986) and Misra et al. (2003) have found that many international students lack the ability to develop deep emotional friendships across cultural groups and with fellow American students for the purposes of social support. Instead, international students more easily develop friendships and seek help from co-national and co-ethnic students and others (Bulthuis, 1986; Leong \& Sedlacek, 1989).

\section{Other Models to Explain Adjustment among International Students}

There are also other models, in addition to stress-coping and social support models, that have attempted to explain relevant adjustment processes in international student samples. Hechanova-Alampay et al. (2002) has evaluated critically various models of adjustment while trying to understand the adjustment of student sojourners including models such as Adler's (1975) U-curve model of sojourner adjustment, the cyclical continuous adjustment model of Anderson (1994), and the discontinuous adjustment model proposed by Golden (1973). According to U-curve model of Adler 
(1975) there are five phases of cross-cultural adjustment, namely, the initial contact, disintegration, reintegration, autonomy, and independence stages. As the titles of the stages are descriptive of specific stages in adjustment processes, initially the acculturating individual loses his or her level of comfort in the middle of the process (i.e., during disintegration and reintegration) and gains back his or her level of comfort at the end of the final phase of the adjustment process. Church (1982) and Black and Mendenhall (1990) as cited in Hechanova-Alampay et al. (2002) reported a lack of methodological rigor and inconsistencies in studies evaluating the U-curve model. They reviewed several past studies that were based on the U-curve model to explain adjustment among sojourners, and found that the five stages of the U-curve model were not consistently represented in every immigrant student and also that the sequencing of the stages was inconsistent. In contrast to this U-curve model, Anderson (1994) has proposed that the adjustment process is an ongoing, cyclical, non-linear, discontinuous process, but studies such as that by Golden (1973) as reported by Hechanova-Alampay et al. (2002), found that the psychological moods of international students changed discontinuously, tended to rise and fall, but were not continuous. After reviewing the methodological limitations of these stage models, Hechenova-Alampay et al. (2002) concluded that more rigorous examination of the underlying processes of adjustment of sojourners should be done to understand the extent to which each of these models can explain the acculturation process.

Over many years, researchers have investigated adjustment among international students in the United States using various models (Cheng et al., 1993; Hayes \& Lin, 1994; Michailidis, 1996; Sadrossadat, 1995). While stress-coping models, social support 
models, and several other models have given useful accounts of adjustment and specific adjustment-related outcomes among international student samples, Sandhu and Asrabadi (1994) found that the adjustment of international students was also influenced by additional acculturation-related factors, such as feelings of racial discrimination, alienation, cultural identity, threats to racial identity and culture-specific values, and feelings of being stereotyped.

This review takes the position that the existing research literature should be supplemented and broadened, so as to include unique acculturation-related variables to understand better the adjustment processes of international students. Acculturation models provide socio-cultural, as well as psychological dimensions of adjustment in immigrants (Nicassio, 1985). Several research studies (e.g., Constantine et al., 2004; Puritt, 1978; Shin, 1994) have examined the adjustment of international students using acculturation models and found this framework to be helpful in organizing and integrating a range of adjustment-related stress variables. The use of an acculturation model can enhance current understanding as it conceptualizes adjustment as a coordinated process that may include multiple dimensions of adjustment in contrast to current conceptual models which are more likely to be narrowly defined and restricted in scope to single or a few specific dimensions of adjustment. An acculturation model compliments current research by integrating multiple research dimensions into a holistic research paradigm. An acculturation model also integrates acculturation-specific cultural variables, which previously have been examined among mainstream immigrants, but not among the international students. 


\section{Acculturation Framework}

The process of acculturation takes place when members from different cultures come in continuous contact with a mainstream, dominant, host group culture (Sam \& Berry, 1995; Sandhu \& Asrabadi, 1994). During this process, immigrants continuously need to reorganize and modify their various sub-identities, including self-identity and cultural identity related to their new, as well as their old culture (Ben-Shalom \& Horezczyc, 2003). In contrast to these uni-dimensional models of acculturation according to which, acculturating immigrants gradually change their cultural orientation from their traditional culture to the dominant host culture, competing bi-dimensional models of acculturation advocate that change occurs along two dimensions, i.e., in their cultural orientation towards their traditional culture, as well as in their orientation towards the host culture. This formulation is distinct from the notion that immigrant groups gradually change on a continuum towards the cultural orientation of the host group, as advocated by uni-dimensional models of acculturation. In the bi-dimensional conceptualization, the dynamics of acculturation are influenced by both the dominant group's culture, and the acculturating group's culture (Flannery, Reise, \& Yu, 2001). In addition to this bidimensional model of changes in the cultural orientations of immigrants, while adopting the host group's cultural practices, immigrants also influence their hosts with their traditional culture that they bring to the host country. Often, some of their traditional cultural practices are adopted by host group members, or the host group culture is modified by the influence of the traditional culture(s) of immigrants (Redfield, Linton, \& Herskovits, 1936, as cited in cited Zagefka \& Brown, 2002). 
Even though change in the host group culture because of interaction with immigrants' native culture is proposed in bi-dimensional acculturation theories, the dominant cultural group potentially has both higher and stronger influences in bidirectional exchanges because of: (a) its greater numerical representation, and (b) the built-in, established nature of their culture. Dominant groups often deliberately or unwittingly force changes in acculturating groups (Sandhu, Portes, \& McPhee, 1996). As a result, the acculturating group is often forced to undergo more intense modification of specific aspects of its traditional culture, resulting in varying degrees of conflict (Nicassio, 1985).

Conflicts between core values, beliefs, and behavioral patterns associated with two autonomous cultures often lead to the experience of stressful, tense psychological states by individuals belonging to one cultural background when they encounter other cultures (Anderson, 1991). As acculturating individuals need to make a number of personal, social, and environmental adjustments during this forced adaptation to the host culture, they often incur significant psychological distress, which can reduce their quality of functioning at multiple levels, including functioning in physical, psychological, and social domains (Sandhu et al., 1996). This, in due course, may lead to the feelings of powerlessness, as well as perceptions of threat to self and cultural identity. Learned helplessness, hopelessness, hostility, perceptions of being alienated, and of being marginalized can all together aggravate this distress (Sandhu et al., 1996). Nwadiora and McAdoo (1996) found that reduced physical and mental health were associated with psycho-cultural stresses that arise during acculturation due to differences in host and traditional cultures. Berry and Annis (1974) labeled this as acculturative stress. 
The attitudes and policies of the host culture have been found to influence the speed of the acculturation process by either facilitating or inhibiting the pace of the process of acculturation (Anderson, 1991). Whether the acculturation process is facilitated or inhibited has been found to be determined by similarities and differences between host and immigrant cultures. As the differences between cultures increase, every aspect of culture such as food, appearance, and social lifestyle seem to be different, and difficulties encountered while adapting to all of these aspects of culture can be sources of strain for immigrants. Significant cultural differences between the host culture and immigrants' traditional culture are potential sources of acculturative stress, and the degree of cultural distance has been found to be strongly and positively correlated with the acculturative stress that is experienced (Nwadiora \& McAdoo, 1996).

The transition of international students into a host culture is a process of acculturation rather than merely a process of psychological adjustment as it involves exposure to the host culture and modification of pre-existing cultural identities parallel to ongoing experiences of adjustment to the college experience (Sakamoto, 2006; Sandhu \& Asrabadi, 1994). Past research has found that the experience of inter-cultural encounters and acculturation occur through sojourns, pleasure travels, voluntary and involuntary migration, colonization or invasion, as well as during education in a foreign country (Church, 1982; Matsumoto, Hirayama, \& LeRoux, 2006; Sakamoto, 2006; Sandhu \& Asrabadi, 1994; Ward, Bochner, \& Furnham, 2001). Adjustment is conceptualized as a short-term social and psychological adaptation of individuals to their situational contexts, while acculturation refers to alterations of various sub-identities of individuals, including their cultural and self-identities. Acculturation involves rigorous modification of self 
concepts, acceptance of host culture values, integration and assimilation of these newly encountered values into one's own traditional cultural values (Sandhu et al., 1996).

The process of acculturation, in general, poses strains and stresses for immigrants as they make gradual transitions into the host culture. While bidirectional models claim that acculturation is an interactive process in which cultural identities of members of both the host and immigrant groups are modified as a result of interactions between immigrants and host group members, immigrants encounter significantly more stresses and strains as they experience more demands for change, modification, and adoption in contrast to the minimal or negligible demands for changes that host group members encounter (Flannery et al., 2001; Nicassio, 1985; Sandhu et al., 1996). As international students are also exposed to all these acculturation factors, they also face acculturation stresses during their transition to the host culture.

Several acculturation-related variables that have been examined in studies of mainstream immigrant populations may also play a significant role in acculturation processes among international students. Hence, the current research study primarily hypothesized that international students undergo acculturation and incur acculturationrelated stresses during their transition into post-secondary education systems in American colleges and universities. It is important to know if the influence of the previously identified acculturation-related factors such as, cultural racism and racially obnoxious situations can be generalized to international student populations. A review of these acculturation-related stress-inducing factors will be made next to gather further support for the position of this research project that the extension of traditional acculturation 
models to international students is appropriate and will result in improved understanding of adjustment outcomes among international students in United States universities.

\section{Cultural Racism}

Cultural racism empowers a dominant cultural group to support their feelings of the appropriateness of social norms related to their own culture (Utsey \& Ponterotto, 1996). Cultural racism has been found to be associated with hostility towards minority groups, as this factor enforces unidirectional acculturation processes rather than openness regarding bidirectional interactive acculturation processes between the immigrant and host groups. This creates more acculturation-related stresses for immigrants. Anderson, in one of his studies on acculturative stresses in an African-American immigrant sample, found that acculturation and adjustment become more difficult in the face of cultural racism if immigrants who belong to a minority group have norms that vary significantly from those of the dominant cultural group (Anderson, 1991). Immigrants' adjustment is made significantly more stressful by instances of indifference and hostility of members of the host culture group as result of cultural racism. It might also be true that international students experience cultural racism as cultural racism, prompting perceptions that the dominant American culture is an appropriate norm and all the other cultural practices are deviations from this norm. Many international students engage in cultural practices that are significantly different from those which are normative in the United States. Perceptions of the inappropriateness of their traditional cultures and the perception of forced attempts to modify their behavior lead to experiences of significant levels of stress and distress. 


\section{Racially Obnoxious Situations}

Sutherland and Harrell (1986) have found that certain situations that are racially obnoxious may lead to stressful experiences because of the influence of racial factors on those situations. Social psychological research has found that simple everyday interactions between African-Americans and Caucasians were associated with increases in physiological arousal of both of them (Anderson, 1991; Porier \& Lott, 1967; Vidulich \& Krevanich, 1966). Subjective feelings of apprehension were also found to be associated with situations where an individual member of a minority group encounters members of a dominant racial or ethnic group (Anderson, 1991). This was found to be more prevalent in situations where the minority group to which a specific individual belonged was underrepresented numerically in the general population. This might be due to perceived lack of sufficient co-ethnic social support. While previous studies related to racially obnoxious situations did not specify clearly if the samples included in the studies were recent immigrants or American citizens, conceptual variables that contribute to the generation of racially obnoxious situations are inherent in the daily experiences of racial minority groups and recent immigrants who undergo acculturation fall into these racial or ethnic minority groups. Hence, research findings regarding the psychological impact of racially obnoxious situations may generalize to many immigrant populations, including international student populations.

\section{Collectivism vs. Individualism}

The United States is generally viewed as maintaining values associated with individualism, which is characterized by social assertiveness, independence, and selfreliance (Constantine et al., 2004; Markus \& Kitayama, 1991). In contrast, collectivistic 
cultures emphasize the importance of social relations and social support seeking behaviors among their members. As members of collectivistic cultures encounter the individualistic attitudes and traditions of American society and lack the social support networks present in either their host or home country, they may experience increased feelings of acculturation-related stress. As there are significant disparities between the general individualistic and collectivistic traditions, this factor might influence significantly acculturation processes experienced by persons who come from societies where collectivistic values are fostered. Acculturation to the contemporary United States may result in significant acculturative stress and social support seeking may be adopted as a coping strategy to lessen the acculturation stress experienced by individuals who come from more collectivistic cultures. If social support resources are compromised or inaccessible, coping with acculturative stress may be substantially more difficult, and successful acculturation-related outcomes may be more difficult to achieve. Many international students in colleges and universities in the United States come from largely communal or collectivistic cultures (e.g., cultures found in Africa, Asia, and Latin America) that are characterized by connectedness to socially important others and a strong valuing of interpersonal relationships (Constantine et al., 2004). Predominant emphases within United States culture on individualism and autonomy may further increase international students' perceived stress related to other co-occurring factors. International students from Europe were found to experience less acculturation stress compared to international students from Asia (Yeh \& Inose, 2003). This finding might be the result of similarities among individualistic cultural attitudes among European and American cultures, in contrast with more collectivistic East Asian cultures. 
Comparison of Conceptual Models Highlighting Acculturation or Stress-Coping

Processes

Acculturation models have some important conceptual similarities with stresscoping models. For example, acculturation-focused frameworks provide elaboration about particular cultural contexts of acculturative stress. Similar to stress-coping models, acculturation-focused models hypothesize that increased exposure to, and adaptation, to a host culture exposes immigrant individuals to cultural values and norms present in the host culture that might act as internal, as well as environmental demands that result in stress (Black \& Markides, 1993). Acculturation-focused models conceptualize the negative outcomes of acculturation as being mediated through acculturation-related stresses. Depending on resource availability, acculturating individuals might adopt either problem-focused or emotion-focused coping strategies to cope with acculturation-related stresses.

Although acculturation models are conceptually similar to stress-coping models in some ways, acculturation models also encompass a broader range of dimensions of adjustment, such as social as well as psychological domains. In addition, acculturation models tend to be derived more from sociological rather than psychological disciplinary traditions. While stress and coping models tend to explain psychological aspects of adjustment processes, acculturation models are more likely to include and integrate sociological or cultural factors into hypothesized adjustment processes and focus on broader psychosocial phenomena as relevant adjustment outcomes. Acculturation models tend to be integrative approaches to understand the adjustment of immigrant groups, in contrast to the reductionistic approaches of many stress and coping models. 
While many studies have examined international student samples to identify potential contributing stressors for specific positive or negative outcomes during adjustment processes, they have typically either examined one stressor or one category of stressors per study. However, significant stressors rarely occur alone, but often overlap with one another. Stressors are often experienced as they co-occur in presence of other stressors (Sandhu \& Asrabadi, 1994). Frequently, one stressor triggers another stressor, and a cluster of stressors may develop (Thoits, 1995; Pearlin, 1989). The transitional events model proposed by Felner, Rowlinson, and Terre (1986) conceptualizes that major initial stressor as leading eventually to a series of other stressful events (as cited in Spaccarelli, 1994). Integration of divergent research traditions is clearly needed as further integration of conceptual models and empirical findings may yield a better and more comprehensive understanding of underlying phenomena leading to specific outcomes (Michailidis, 1996). Current research on international students tends to be fragmented and inconsistent as many existing studies have ignored specific stressors that were examined in other studies.

One limitation of the existing research literature is that the current literature is fragmented and there is a need to examine the adjustment of international students using complimentary models that can be used to integrate many of the currently fragmented research findings. In addition to the multiple stressors that have been examined in past research studies, unique acculturation variables such as cultural identity, as well as racial or ethnic factors may influence significantly adjustment processes among international students (Hechanova-Alampay et al., 2002; Sandhu \& Asrabadi, 1994). For example, acculturation-related factors that might induce stress, such as racially obnoxious 
situations, cultural racism, and issues of collectivism versus individualism have been examined among mainstream immigrant populations (Anderson, 1991; Constantine et al., 2004; Markus \& Kitayama, 1991; Sutherland \& Harrell, 1986; Utsey \& Ponterotto, 1996), but few studies have examined these issues in samples of international students. In general, studies on immigrant populations found that factors such as racially obnoxious situations, collectivism vs. individualism influence processes of acculturation and are related to hostility directed toward the acculturating group members, hampering the progress of their acculturation (Anderson, 1991; Constantine et al., 2004; Markus \& Kitayama, 1991). Integrating Concepts of Acculturation into Stress-Coping Conceptual Frameworks Acculturation models also conceptualize adjustment as a process by which cultural demands (similar to internal or external environmental demands of transactional stress-coping models) burden the acculturating individual and result in acculturationrelated stresses. Depending on how acculturating individuals attempt to cope with acculturation-related stressors (i.e., whether they adopt problem-oriented or avoidance coping strategies), either adaptive or maladaptive outcomes are more likely to result. This conceptual parallel between acculturation-oriented and stress-coping frameworks allows researchers to expand current understanding of acculturation-related processes in terms of transactional stress-coping models. Berry (1997) has described the process of acculturation within a stress and coping framework, integrating concepts from both the stress-coping and acculturation perspectives. 


\section{Berry's Four Groups Model}

Based on how individuals attempt to cope with acculturation-related stresses, Berry (1997) classified the outcomes of processes of acculturation into four distinct categories, namely, integration, assimilation, separation, and marginalization. When the individual who is acculturating adapts to the host culture while retaining elements of his or her traditional culture, integration occurs. Adaptation to a host culture without retaining elements of one's traditional culture results in assimilation. In these two scenarios, the individual is assumed to be able to access enough coping resources so as to withstand the stressful experiences related to adaptation to the host culture and broader processes of adjustment (Kosic, Mannetti, \& Sam, 2005; Liem, Lim, \& Liem, 2000).

If an individual retains his or her traditional cultural orientation but fails to adapt to the host culture, separation results. An individual considered to be separated and isolated from the mainstream host culture functionally does not adapt to, or participate in, any of the host culture's processes. If in a few other contexts, the individual fails to retain his or her traditional cultural orientation, in addition to failing to adopt the culture of the host country, the outcome is classified as marginalization (Berry, 1997; Sam \& Berry, 1995; Kosic et al., 2005; Liem et al., 2000). Separation and marginalization result when the individual does not have access to sufficient individual or social resources to cope effectively with specific stressors experienced during ongoing adaptation processes. Individuals may either make insufficient attempts to cope with stressors encountered or they may adopt avoidance coping mechanisms. Research has found that immigrants who are classified as separated or marginalized report higher levels of stress compared to individuals who either integrate or assimilate to the host culture. 
Berry (1997) found that cross-cultural contacts often bring about negative psychological consequences and that these negative psychological consequences have been found to predict significant acculturative stress. Acculturation stress can be evaluated using a traditional stress-coping model and acculturation-related outcomes can also be classified into problem-oriented and avoidance coping styles. Problem-oriented coping styles often result in positive outcomes of acculturation-related processes, such as integration or assimilation, while avoidance coping styles might result in negative acculturation outcomes, i.e., separation or marginalization (Berry, 1980; Berry, 1997; Handelsman, Gottlieb, \& Knapp, 2005; Kenkel, Deleon, Mantell, \& Steep, 2005). The Relation of Acculturation Processes to Alcohol Use Patterns

Many research studies (e.g., Chen, Unger, Cruz, \& Johnson, 1999; Vega, Gil, \& Zimmerman, 1993) have found that negative health risk behaviors, such as smoking and alcohol use, increase as acculturation levels increase in general immigrant populations. Research by Caetano (1987), as well as Caetano and Medina Mora (1988) suggested that acculturation modifies alcohol use behaviors among immigrants. Many researchers have tried to document factors that underlie the development of alcohol use behaviors in immigrant populations. Based on past research, the relation of alcohol use to acculturation processes can be linked to three important factors, (a) the accepting environment (i.e., social norms) of the United states toward alcohol use; (b) alcohol is used as a social lubricant during the adjustment phase; and, (c) acculturation results in acculturative stress and alcohol use and abuse behaviors might be adopted as avoidance coping strategies to cope with this stress. 
More accepting social norms. According to Hsia and Spruijt-Metz (2003)

smoking, alcohol, and substance abuse behaviors are not as accepted in many cultures as they are in contemporary American culture. The accepting environment in the United States is a significant risk factor and it may increase the probability that these risk behavior patterns will be adopted by international student populations. Social influences such as the pro-smoking, pro-alcohol use related social norms of the United States and the social acceptability of health risk behaviors in United States society also have been found to influence alcohol use behaviors among adolescent immigrants (Guilamo-Ramos, Jaccard, Johansson, \& Tunisi, 2004), including international students (Unger et al., 2000). In addition, positive expectancies including those that assert that alcohol is a stress reliever, or that it provides pleasure are more prevalent in the United States. These alcohol expectancies are additional significant risk factors that have been found to increase the likelihood of alcohol use behaviors in samples of immigrants.

Alcohol expectancies develop through peer group modeling, advertisements, and direct or indirect experience with alcohol use and these influences may vary in different cultures (Velez-Blasini, 1997; Christiansen, Goldman, \& Inn, 1982; Christiansen, Smith, Roehling, \& Goldman, 1989). The phrase peer modeling is used here to represent the social exposure to the behavior of peers, and the perceptions of international students of peers' drinking behaviors rather than modeling the behavior of peer group members, similar to constructs delineated in Badura's Social Learning Theory.

As individuals become more and more acculturated, these expectancies are learned thorough increased exposure to the social influences of peer models from the host culture. Mallinckrodt, Shigeoka, \& Suzuki (2005) collected data from international 
students from Asian Pacific Islands and found that individuals who were more acculturated identified themselves more with core values reflecting Western cultural attitudes, values and beliefs. As one becomes more acculturated into a new society, cultural adaptations will expose the individual to risk and protective factors for alcohol use or other health risk behaviors that are prevalent in the host society (Unger et al., 2000). The availability of alcohol and the acceptability of alcohol use behaviors in United States society are high, in contrast to many international students' home countries. Therefore, as international students get more acculturated, they will have more access to alcohol and they are more likely to engage in alcohol use behaviors given the semirestrictive nature of mores surrounding alcohol use by youth in the United States.

Alcohol as a social lubricant. Alcohol is widely considered to enhance social interactions, i.e., to function as a social lubricant (Caetano, 1987) and immigrants might use alcohol to get closer to, or to imitate host culture group members during acculturation. In most college and university settings in the United States, heavy alcohol use and its use in social settings among students are normative risk behaviors on campus (Bennette, Smith, \& Nugent, 1991). International students may adopt alcohol use behaviors in these settings as social lubricant to get closer to native peer group students. Alcohol use as an avoidant coping strategy. Few past research studies conducted on mainstream immigrant populations have reported that alcohol was used as an avoidance coping strategy to reduce perceived stresses related to acculturation. While past research has found that acculturative stress was related significantly to alcohol use behaviors (Caetano, 1987; Gilbert \& Cervantes, 1986; Johnson, Gruenewald, Treno, \& Taff, 1998), there were, however, inconsistencies regarding the strength and sign of the 
correlations between acculturative stress and alcohol use behaviors. Studies such as that conducted by Smith (2000) suggest that acculturation level was negatively correlated with alcohol use, while other studies (Balcazar, Peterson, \& Cobas, 1996; Gil et al., 2000; Lovato, Litrownik, Elder, Nuñez-Liriano et al., 1994) have found positive correlations between acculturation level and alcohol use behaviors. Adding to this inconsistency, a study by Barrett, Joe and Simpson (1991) found no significant correlation between these variables. While important theoretical and methodological concerns contribute to these variations in research findings, factors such as the characteristics of study populations including: age, gender, and ethnicity may also have contributed significantly to differences in research results. Studies using different populations often documented different results.

Studies that have used different ethnic groups in their samples have found different relations between acculturative stress and alcohol use variables in terms of either the sign, strength of the correlation or both. Velez-Blasini (1997), reported that there are also differences across various subgroups within Hispanic ethnic groups, in terms of alcohol use patterns. As the inherent characteristics of each of acculturating groups vary from each other, this variation might be an expectable result. This might also be true across different samples with participants of different age groups. In addition, various immigrating groups may have different norms for alcohol use acceptability in accordance with age or other factors. This suggests the need to examine the sign and strength of parallel bivariate correlations among international student samples. 
However, studies by Chen et al. (1999) and Vega et al. (1993) found that negative health risk behaviors, such as smoking and alcohol use, increase as acculturation levels increase, irrespective of group differences in general immigrant populations. Increasing similarities in negative risk behavior patterns, despite diversities in their native cultures might be related to the strong influences of the host culture compared to the diminishing influences of immigrants' native cultures. Increasing alcohol use may be a response to both acculturation-related processes, as well as specific social influences. Although social influences can explain a portion of the variance in alcohol use outcomes, it can be argued that social influences also increase as an individual gets more and more exposed to the host culture, and this exposure increases only after one gets more and more acculturated.

International students, who come to the United States seeking higher education also encounter most aspects of the host culture and undergo a process of acculturation in many ways similar to that experienced by their mainstream immigrant counterparts (Sakamoto, 2006; Sandhu \& Asrabadi, 1994; Ward, Bochner, \& Furnham, 2001). International student populations are similar to mainstream immigrants in the ways they also undergo parallel acculturation processes, and are exposed to similar cultural contexts of a host culture. This methodological similarity may support the application of acculturation-related conceptual models to examine international students' adjustment. However, international students vary from mainstream immigrant population in terms of the racial/ethnic diversity of their populations, age differences, and the additional academic transition-related stressors they face, which generally are not experienced during mainstream immigrant populations' acculturation processes. 
While methodological similarities support the use of similar conceptual frameworks, substantial diversity among international students emphasize practical barriers to the simple application of such models. It is important to document the sign and strength of correlations between acculturation processes and alcohol use among international students. Few researchers have examined college student populations of international students to document their acculturation processes and associated negative health risk behaviors. For example, Unger, Trinidad, Weiss, and Rohrbach (2004), in their study of Asian-American adolescent immigrant students at one university in California, found that acculturation in the context of American culture was associated significantly with drug and alcohol use. Hendershot, MacPherson, Myers, Carr and Wall (2005) examined international college students who came from Asian countries and found that the level of acculturation was significantly positively correlated with levels of alcohol use. However, there were no other studies to support whether these research findings on Asian student populations are generalizable to other groups of international students.

There are likely to be many significant group differences within the broader international student population with regard to alcohol use patterns, depending on these students' country of origin, as the socio-cultural norms related to alcohol use, alcohol acceptability, and availability vary significantly across cultures. Unger et al. (2000) reported that research shows group differences in terms of the intensity of alcohol use behaviors across international students and Makimoto (1998) found that students who came from the Asian Pacific Islands, showed the lowest alcohol use patterns compared to 
other ethnic groups of students. As international student populations are extremely diverse populations, the generalizability of these results is questionable.

\section{Future Research Directions}

Based on gaps identified in the current research literature on international students, two broad recommendations can be made. First, to apply acculturation-related conceptual models to understand more clearly the adjustment of international students, specifically, with regard to both adaptive and maladaptive adjustment outcomes. Second, to examine significant influences upon the alcohol use behaviors of international students.

Examination of international student samples using an acculturation paradigm is important for two reasons. First, international students may experience acculturation processes in similar ways as mainstream immigrants and it is reasonable to adapt an acculturation paradigm to understand their acculturation-related experiences. Second, the generalizability of mainstream immigrant research to international students may be questionable because of significant differences between international students and mainstream immigrants in terms of the diversity of international student populations. For example, the academic demands experienced, the demands for language proficiency and typical social supports received during adjustment may differ significantly from the experiences of mainstream immigrant populations. Factors such as the unique types of

peer and family supports that international students receive during acculturation (Misra \& Castillo, 2004; Sue, 1999), and higher demands for language proficiency (Poyrazli et al., 2001) contribute to significant differences in the experiences of international students as compared to mainstream immigrant populations. International students also may vary 
from mainstream immigrant population substantially in terms of their racial/ethnic diversity and the age ranges within their populations. In addition, international students face demanding academic transition stressors which are not normatively experienced during mainstream immigrant populations' acculturation processes.

Again, there are two important reasons for the recommendation to examine alcohol use behaviors among international students. First, as past research has documented significant relations between acculturation processes and alcohol use behaviors in immigrant populations, it is important to determine if that relation can be generalized to the international student population. Second, as the majority of international students belong to late adolescent or young adult age groups, they are vulnerable to exposure to risk behaviors such as peer drug and alcohol use and abuse behaviors (Catanzaro, \& Laurent, 2004; O’Mally, Johnston, \& Bachman, 1998).

Past research has identified three important factors that explain in part the relation between acculturation and alcohol use, (a) the accepting environment of the United States toward alcohol use (Guilamo-Ramos et al., 2004; Hsia \& Spruijt-Metz, 2003; Unger et al., 2000); (b) alcohol use as a social lubricant (Benette, 1991; Caetano, 1987); and, (c) acculturation resulting in acculturative stress and the use of alcohol as an avoidance coping strategy (Caetano, 1987; Gilbert \& Cervantes, 1986; Johnson, Gruenewald, Treno, $\&$ Taff, 1998).

However, there is inconsistency regarding the strength and sign of bivariate correlations between acculturative stress and alcohol use behaviors across different immigrant samples. Studies using different populations often have found widely varying results (Balcazar et al., 1996; Barrett et al., 1991; Gil et al., 2000; Lovato et al., 1994; 
Smith, 2000). Characteristics of study populations such as, age, gender, ethnicity, and different norms for alcohol use acceptability may contribute significantly to differences in research results. This issue suggests the need to examine the sign and strength of parallel correlational relations among international student samples.

\section{The Current Study}

The proposed study is designed to extend the existing literature by documenting acculturation-related processes, alcohol use behaviors, and relations between the two constructs within a sample of international students. The current study hypothesizes that acculturation-related processes may increase the likelihood of alcohol use and abuse behaviors among international students. In addition, it is hypothesized that three separate factors partially mediate relations between levels of acculturation and alcohol use behaviors among international students. The term partial mediation is used in place of the term full mediation to convey the meaning that the relation between acculturation and alcohol use is potentially mediated by multiple variables and each of those variables is partially responsible for the variation in the dependent variable.

The three separate constructs hypothesized to partially mediate this relation are (a) acculturative stress, (b) perceived peer modeling of alcohol use, and (c) perceived injunctive social norms for alcohol use among university student peers. An exploratory hypothesis will determine if these relations are similar in groups of international students reporting high or low levels of social support among international students. As international students undergo processes related to acculturation, they may experience acculturative stress. Alcohol use behaviors may be more likely to follow the experience of increased acculturative stress, as an avoidant coping strategy. Perceived peer alcohol 
use is proposed as a significant mediator of the relation between acculturation and alcohol use because higher levels of acculturation are likely to promote exposure to peer modeling. In addition, the acculturation-related processes are likely to increase international students' exposure to injunctive social norms for alcohol use among university students. Four major hypotheses tested in the current study were

1. Higher scores for indices of acculturation predict significantly higher scores for alcohol use among international students.

2. Acculturative stress partially mediates the relation between acculturation levels and alcohol use among international students.

3. Perceived peer modeling of alcohol use partially mediates the relation between acculturation levels and alcohol use among international students.

4. Perceived social norms of the host university partially mediate the relation between acculturation levels and alcohol use among international students.

A secondary goal of the study included examination of the potential moderating influence of social support on hypothesized relations in the structural model evaluated (i.e., between the levels of acculturation, putative mediators, and alcohol use. It was hypothesized that among international students reporting low levels of social support, (a) acculturation levels would be related more strongly to acculturative stress, and (b) that acculturative stress would be related more strongly to alcohol use. 


\section{CHAPTER 3: METHODS}

\section{Participants}

Table 1 summarizes the demographics of the sample $(N=226)$ in terms of ethnicity, gender, year in school, marital status, number of children, length of stay in the United States, and other key variables. There were 131 (58\%) males and $95(42 \%)$ females in the sample recruited for the current study. Participants were predominantly $(58.9 \%)$ graduate students who were pursuing either masters or doctoral degrees at Florida International University. In terms of ethnicity, Hispanic/Latino students composed $29.2 \%$ of the sample and Asian/Pacific Islander students constituted another $28.3 \%$ of the sample. While $18.6 \%$ of the participants were White/Caucasian students, Black/African Americans students made up $8.4 \%$ of the sample, and another $15.5 \%$ of the participants reported that they belonged to other ethnic groups. The participants' ages ranged from 19 to 42 years old, with an average age of 25.9 years ( $S D=4.4$ years). Most $(78.8 \%)$ of the students in the sample were single, but $19.5 \%$ of them were married and $1.8 \%$ of them were divorced. Only $6.6 \%$ participants in the sample reported being the parent of one or more children.

The international students in the sample reported their home countries as India ( $n$ $=32,14.2 \%)$, Venezuela $(n=15,6.6 \%)$, Trinidad $(n=14,6.2 \%)$, China $(n=12,5.3 \%)$, Jamaica $(n=12,5.3 \%)$, and Colombia $(n=11,4.9 \%)$, while another 130 participants $(57.5 \%)$ reported a wide range of other home countries. The largest subgroup in the sample (44.2\%) reported their religious affiliation as Christian, while other groups of participants reported Hinduism (13.3\%) or Islam (4.9\%) as their religious affiliation. With regard to living arrangements, the majority of the participants reported living off- 
campus $(89.8 \%)$. The largest subgroup $(n=81,35.8 \%)$ reported living with family members or parents, while smaller groups reported living alone $(n=54,23.9 \%)$, living with friends from their native country $(n=50,22.1 \%)$, living with other international students $(n=23,10.2 \%)$, or living with American friends $(n=18,8.0 \%)$. Measures

All participants were assessed with regard to: their acculturation levels using an adapted version of the Short Acculturation Scale for Hispanics (SASH, Marín, Sabogal, VanOss Marín, Otero-Sabogal, \& Pérez-Stable, 1987); their acculturative stress levels using the Acculturation Stress Scale for International Students (ASSIS, Sandhu \& Asrabadi, 1994); their alcohol use patterns using the Alcohol Use Disorders Identification Test (AUDIT, World Health Organization, 1992); their perceptions of peer alcohol use with an adapted version of Daily Drinking Questionnaire (DDQ, Collins, Parks \& Marlatt, 1985); and perceived injunctive social norms for alcohol use using an adapted version of five items measuring perceived norms developed by Perkins and Berkowitz (1986).

Short Acculturation Scale for Hispanics. The Short Acculturation Scale for Hispanics (SASH) was developed by Marín, Sabogal, Marín, Otero-Sabogal, and PérezStable (1987). The SASH was developed to identify reliably levels of acculturation in Hispanic populations and classify the respondents as high or low on self-reported acculturation level. The scale consists of 12 items related to three factors, namely, (a) Language Use, (b) Media, and (c) Ethnic Social Relations. Sample items include "In general, what language do you read and speak?" (Language Use), "In what language are the TV programs you usually watch?" (Media), and "you prefer going to social 
gatherings/parties at which people are.” (Ethnic Social Relations). Respondents responded to each statement on a scale with five response categories, Only Spanish (1), More Spanish than English (2), Both Equally (3), More English than Spanish (4), and Only English (5). An average score of 2.99 was suggested by the authors as a cut-off to differentiate low versus high acculturated respondents.

An adapted version of this scale was used in the current study with modified response categories where the word "Spanish" was replaced with the phrase "your native culture/language." This modification was warranted because international students in the current study were expected to include individuals from diverse cultural and linguistic backgrounds, rather than limited to Spanish language/Hispanic or Latino ethnicity. Numerous research studies report that the SASH is a reliable and valid measure for a wide range of Hispanic populations including: Mexican-Americans, Cuban-Americans, Puerto Ricans, Dominicans, and Central and South Americans (e.g. Barona \& Miller, 1994; Felix Ortiz, Newcomb, \& Myers, 1994; Vega, 1992). Marin et al. (1987) reported a Cronbach's alpha level of .92 for the SASH. In addition, Yo (2006) reported that adapted and extended versions of this instrument developed for ethnic groups other than Hispanics/Latinos, or specific age groups of immigrants have been developed by researchers and that they have acceptable levels of reliability and validity. For example, Barona and Miller (1994) reported that the SASH-Y (an adapted youth version of SASH) has excellent internal consistency (.94), with alpha coefficients of .92 for Hispanics and .85 for non-Hispanic samples. In addition, they reported an odd-even split half reliability of .96. In the current study, the SASH had a Cronbach's alpha of .88. 
Acculturative Stress Scale for International Students (ASSIS). The ASSIS was developed by Sandhu and Asrabadi (1994) and was used in the current study to assess international students' levels of acculturative stress. This measure included 36 Likerttype items that respondents rated from strongly disagree (1) to strongly agree (5). The ASSIS consists of seven subscales, including: perceived discrimination (8 items), fear (4 items), homesickness (4 items), stress associated with culture shock (3 items), perceived hate (5 items), guilt ( 2 items), and other nonspecific concerns (10 items). Total scores on the ASSIS range from 36 to 180, and higher scores indicate that higher levels of acculturative stress are experienced by respondents. Sample items on each of the subscales include statements such as: "Many opportunities are denied to me" (Perceived Discrimination); "I feel sad leaving my relatives behind" (Homesickness); "People show hatred towards me" (Perceived Hate); "I feel insecure here" (Fear); "I feel uncomfortable to adjust to new foods" (Culture Shock/Stress due to Change) and "I feel guilty that I am living a different lifestyle here" (Guilt).

Sandhu and Asrabadi (1998) reported high Cronbach alpha coefficients ranging from .87 to .95 in several samples and a Guttman's split-half reliability of .94 which indicate high levels of reliability for the scale. The current study estimated Cronbach's alphas of the ASSIS. In the current study, the ASSIS had a Cronbach's alpha of .93.

Alcohol Use Disorders Identification Test (AUDIT). The AUDIT is a widely used screening instrument for detecting harmful patterns of alcohol consumption. It was developed by a WHO collaborative project (Saunders, Aasland, Babor, de la Fuente, \& Grant, 1993). The AUDIT provides a simple method for the early detection of harmful alcohol use patterns for use in primary health care settings and is the first instrument of 
its type to be derived on the basis of a cross-national study. The AUDIT contains 10 selfreport items which assess respondents in three domains of alcohol use including: Hazardous alcohol consumption, abnormal drinking behavior, and alcohol-related problems. Responses to each question are scored from 0 (never) to 4 (daily or almost daily), resulting in a maximum possible score of 40, with higher scores indicating higher levels of drinking and alcohol-related problems.

The domain of alcohol consumption assesses the quantity of alcohol consumed by the participant to determine whether it is hazardous, and includes questions related to binge drinking, defined as five or more drinks in a row for men and four for women. The domain of abnormal drinking behaviors includes items that determine if the participant meets at least one diagnostic criterion for alcohol dependence disorder. According to the DSM-IV manual, alcohol dependence is defined as maladaptive patterning of alcohol use, leading to clinically significant impairment manifested by at least three or more of seven criteria listed. Last, the domain of alcohol-related problems assesses difficulties and traumas resulting from drinking alcohol, as well as the concerns of family, friends, and health professionals related to respondents' alcohol use behaviors.

Sample items for each of the AUDIT subscales include questions such as: "How often do you have drinks containing alcohol?" (Hazardous Alcohol Consumption); "how often during the past year have you been unable to stop drinking once you started?" (Abnormal Drinking Behavior); and "Have you or someone else been injured as a result of your drinking?" (Alcohol-Related Problems). Rumpf, Hapke, Meyer and John (2002) reported moderate Cronbach alpha coefficients (.75) for the AUDIT. Chung et al. (2000) found that the AUDIT has good sensitivity and specificity for identifying the presence of 
problematic patterns of alcohol use and alcohol-related problems in adolescent populations. The AUDIT has been reported in several research studies as a valid and reliable measure of alcohol-related problems in adolescent populations (see Allen, Litten, Fertig, \& Babor, 1997; Knight, Sherritt, Harris, Gates, \& Chang, 2003; Miles, Winstock, \& Strang, 2001). In the current study, the AUDIT had a Cronbach's alpha of .76. While the current study excluded the Consequences section of the AUDIT, scale items retained for the analyses had a Cronbach's alpha of .84 . The reason for discarding the Consequences items from analyses in the current study will be discussed further in Chapter 5.

Perceived peer norms for alcohol use. Perceived peer norms for alcohol use were assessed using an adapted version of the Daily Drinking Questionnaire (DDQ; Collins, Parks \& Marlatt, 1985). This adapted questionnaire measures participants' perceptions of their peers' alcohol use patterns with respect to various reference groups. This measure includes items that assess the perceptions of international students regarding "typical" and "average" alcohol use behaviors of other international students, as well as host group university students. This questionnaire asks participants to rate their perceptions regarding alcohol use behaviors of their reference groups in terms of both frequency and quantity of use.

The Daily Drinking Questionnaire was developed by Collins et al. (1985) to allow researchers to estimate peers' mean levels of alcohol use during a typical day and week. It has been used extensively in past research and has shown acceptable levels of reliability and validity. Several past researchers have published extended/adapted versions of the DDQ [see the Drinking Norms Rating Form (DNRF) by Baer, Stacy, and 
Larimer, (1991)] and reported good reliability and validity. For example, Lewis and Neighbors (2004) have reported an $\alpha$ of .73 for their sample, while Carey, Borsari, Carey, and Maisto (2006) reported one-month stability coefficients $(N=122)$ for norm estimates that were $.75, .63$, and .65 respectively. A Cronbach's alpha of .71was calculated for the specific adapted version of the scale used in the current study.

Sample items in this version of the questionnaire include: "How many drinks does an average university student drinks?", and "How often do they drink?" The response format uses a 5-point Likert scale where the anchors represent once a day (5), three or four times a week (4), once or twice a week (3), About once a month (2), and less than once a month (1), for the frequency question, and 7-8 drinks (5), 5-6 drinks (4), 3-4 drinks (3), 1-2 drinks (2), and 0 drinks (1), for the quantity question.

Perceived injunctive social norms. Perceived injunctive social norms for the alcohol use of the participants was assessed using a five-item questionnaire described by Perkins and Berkowitz (1986). This measure has been used extensively in past research (e.g., Carey et al., 2006; Wood, Read, Mitchell, \& Brand, 2004). Carey et al. (2006) reported significant 1 -month stability coefficients $(r=.35, p<.001 ; N=122)$, as well as acceptable levels of test-retest reliability (e.g., $88 \%$ of the retest estimates were within 1 point of the original). The five statements range from extremely conservative (drinking is never a good thing to do) to extremely liberal (a frequent 'drunk' is okay). Participants select the statement that best represents (a) his/her personal attitude, and (b) perceived social norms. For the current study, participants were required to report perceived norms for other international students at FIU, in addition to the two sets of 5 statements described above developed by Perkins and Berkowitz (1986). 
Social Support Questionnaire 6 (SSQ 6). The Social Support Questionnaire (SSQ) is a widely used instrument for measuring appraisal of available social support. The 27-item SSQ was developed by Sarason, Levine, Basham, and Sarason (1983). Each item on the scale describes a challenging situation in which social support may offset stress in that situation. The SSQ assesses two aspects of participants' appraisal: (a) the number of available others on whom the individual can count in that context (i.e., the Nfactor), and (b) the individual's degree of satisfaction with the available number of social supports (S-factor) for that specific context. Each item uses a 6-point Likert scale, from very dissatisfied (0) to very satisfied (5). Sample items include the statement, "Whom can you really count on to distract you from your worries when you feel under stress?"

Sarason et al. (1983) reported an $\alpha$ coefficient of .97. Test-retest stability coefficients over a 4-week interval were .90 and .83 for the $\mathrm{N}$ and $\mathrm{S}$ scores, respectively. An abbreviated, 6-item form of the SSQ (i.e., the SSQ-6) was developed by Sarason, Sarason, Shearin, and Pierce (1987) and was used to estimate the social support appraisals by international student participants in the current study. The SSQ-6 was used for two reasons: (a) lengthy assessment might be a barrier for data collection (Sarason et al., 1987), and (b) strong evidence for psychometric equivalence between the original and abbreviated forms. Sarason et al. (1983) conducted a study to determine the equivalence of SSQ and SSQ-6 scores and they found that there were no significant differences between scores of the SSQ-6 and the original SSQ. They also compared correlations among a variety of specific measures and concluded that the SSQ-6 is an adequate substitute for the original SSQ. In the current study, the SSQ-6 questionnaire had a Cronbach's alpha of .88 . 
Other outcome measures. International Students Scholastic Services (ISSS) at FIU endorsed the current study. The Associate Director of ISSS, Mr. Ted Randell, allowed Mr. Kanaparthi to have access to the email list-serve used by ISSS to communicate with all international students at the university in return for some information related to a few additional outcome variables (e.g., educational outcomes) that were collected from the participants. These additional data may help ISSS to enhance their supportive services to international students by addressing the specific issues that international students identified as particularly problematic. Hence, in addition to the alcohol use behaviors assessed in the current cross-sectional study, several more outcomes related to academic performance, health insurance for international students and immigration issues were measured. These outcomes are measured primarily by single items designed to assess specific issues. While the data generated by these additional questions were not used to test the four major hypotheses of the proposed study, these items were summarized to provide descriptive information to ISSS. These items were not based on any established measures, but were created specifically for the current study.

\section{Procedure}

A web-based survey administration strategy was used to collect data from participants. All questionnaires were assembled on Experimetrix, a web-based survey tool used to schedule and track experiments. Experimetrix is used extensively by researchers in the Department of Psychology at Florida International University. When all questionnaires were compiled on the Experimetrix system, e-mail participation invitations were sent to a listserve of e-mail usernames of all international students at FIU through the Experimetrix system. Prior permission and access was obtained from 
administrators at the International Students Scholastic Services (ISSS) Department at FIU to the international student e-mail listserve. A brief explanation of the purpose and significance of the project, as well as the anticipated benefits to the international student community were included in a cover letter co-signed by the Primary Investigator and an official from ISSS along with a link to access the survey on the Experimetrix system. Each participant was asked to read and approve a letter of informed consent prior to beginning the online questionnaire.

Once the participant completed the questionnaire, information was aggregated with other survey data collected previously in a database by the Experimetrix system which was used later for data analyses. Experimetrix provides options for identifying duplicate submissions by tracking either the email ID of participants or the IP address of the computer used by participants to submitted surveys. No incentives were provided to participants in the study, except for extra credit to undergraduate students who were registered in psychology classes because the Experimetrix system had the facility to provide extra credits for undergraduate psychology students for research participation.

Analytic Plan

The objective of this cross-sectional research study was to examine relations between acculturation-related processes and alcohol use behaviors among international students, including an examination of the potentially moderating influences of age and gender on those relations. The research questions investigated included: If acculturation levels among international students were associated with high levels of alcohol use behaviors; and if so, whether acculturative stress, peer modeling, and/or perceived social norms mediated relations between acculturation and alcohol use among international 
students. In addition, the study examined whether there are any age-, social support-, or gender-related group differences in terms of the strength of the hypothesized relations.

The sample for the study included international students who attended Florida International University (FIU) in Miami, who were diverse in terms of age, gender, ethnicity, country of origin, and native language spoken. Thus, the sample is likely to be representative of an international student population located in other diverse multicultural university communities, but the generalizability of finding from the current study may be limited to similar multicultural universities. Levels of acculturation, acculturative stress, alcohol use behaviors, perceived peer alcohol use, and perceived injunctive social norms for alcohol use were the major variable sets that served as the key measures used in analyses.

This study addressed the major research question by testing four hypotheses:

1. Higher scores for indices of acculturation predict significantly higher scores for alcohol use among international students.

2. Acculturative stress partially mediates the relation between acculturation levels and alcohol use among international students.

3. Perceived peer modeling of alcohol use partially mediates the relation between acculturation levels and alcohol use among international students.

4. Perceived social norms of the host country partially mediate the relation between acculturation levels and alcohol use among international students.

A secondary goal of the study included examination of the potential moderating influence of social support on hypothesized relations in the structural model evaluated (i.e., between the levels of acculturation, putative mediators, and alcohol use). It was 
hypothesized that among international students reporting low levels of social support, (a) acculturation levels would be related more strongly to acculturative stress, and (b) that acculturative stress would be related more strongly to alcohol use.

\section{SEM and AMOS}

Structural equation modeling (SEM) was implemented to test the proposed causal model with latent variables (see Figure 1). SEM is an extension of the general linear model which allows the testing of multiple regression equations simultaneously. Initially, the model was based on the proposed hypotheses and then the measurement of constructs using several reliable and valid measures was established. Later, data collected were fit to the proposed model and overall model fit statistics were examined to determine the adequacy of the fit of the model.

In SEM analyses, independent variables are called exogenous variables and mediating and dependent variables are called endogenous variables. Manifest or observed variables (represented by the rectangles or squares) are the variables that are measured and the latent or unobserved variables (represented by ovals or circles) are those which are not measured directly but inferred from relations among measured variables. Single headed arrows depict hypothesized causal relations.

Three different indicators of acculturation measured by the SASH served as observed measures/indicators of the latent Acculturation construct. Seven different variables derived from the ASSIS scale served as indicators of the latent construct Acculturative Stress. Similarly, other latent constructs, e.g., for alcohol use, perceived injunctive social norms for alcohol use and perceived peer alcohol use were indicated by observed scores for the appropriate instruments described above. 
Multi-group solutions were sought to determine the potential moderating effect of gender on the hypothesized structural model.

Use of AMOS. The fit of the model was evaluated via AMOS 17.0 using the raw data as input and a Maximum Likelihood (ML) solution. The model was examined for statistical identification.

Outliers. Before conducting the primary analyses, the data were evaluated for both univariate and multivariate outliers in a non-model-based approach, using leverage indices for each individual case, i.e., by defining an outlier as a leverage score four or more times greater than the mean leverage score. In the case of outliers, the accuracy of the coding was checked to identify potential coding errors. An additional set of outlier analyses was conducted using model-based outlier analyses. In the case of outliers, they were examined to determine if they were inconsequential or influential for the analyses, by running analyses with and without the identified outliers. In the case of consequential/influential outliers, two strategies may be used: (1) to identify influential cases and making a judgment to keep or delete them, or (2) the adoption of robust statistical techniques to deal with the outliers.

Non-normality. Normality was evaluated using Mardia's index. Univariate, as well as multivariate indices of skewness and kurtosis were evaluated with a criterion that absolute values greater than 2 were identified as significant kurtosis. In the case of any significant non-normality of project data, bootstrapping was performed with 2000 bootstrap replicates.

Missing data. The amount of missing data for each variable was documented. Data were examined to determine if missing data were missing completely at random 
(MCAR), missing at random (MAR), or non-ignorable. Missing data bias was assessed by computing a dummy variable that reflects the presence of missing data and correlating that dummy variable with other variables in the model, as well as an array of demographic variables. Given non-systematic sources of missing data, the expectationmaximum method with the importance re-sampling strategy or a full information maximum likelihood (FIML) approach to missing data as implemented in AMOS 6.0 was used to accommodate missing data.

Model fit indices. Data analyses produced measures from three classes, (a) absolute fit, (b) fit with a penalty function for lack of parsimony and (c) relative fit. In addition to relying on global fit indices alone, (a) modification indices, (b) standardized residuals, and (c) Heywood cases also were evaluated. A variety of indices of model fit evaluated included: the overall chi-square test for model fit, root mean square error for approximation (RMSEA), comparative fit index (CFI), and the global fit index (GFI). The inspection of residuals and modification indices (MIs) was done to identify the presence of significant points of ill-fit in the initial hypothesized SEM model. 


\section{CHAPTER 4: RESULTS}

The results of the current study are presented in three major parts. First, descriptive statistics are presented to describe sample statistics for key variables involved in analyses. Second, bivariate inter-correlations among key variables are presented. Third, the results of structural equation modeling (SEM) analyses are summarized.

\section{Descriptive Statistics}

Table 2 summarizes acculturation-related variables measured by the SASH (Marin et al., 1987) reported by this sample of international students attending FIU. The participants reported a mean total score of $14.38(S D=5.82)$ on the Language Acculturation subscale of the SASH (5 items) out of a possible score of 25 , where 1 represents minimum acculturation and a score of 25 represents the maximum level of acculturation. The participants also reported a mean total score of $12.37(S D=2.68)$ on the Media Acculturation subscale of the SASH (3 items) out of a possible score of 15 , where 1 represents minimum acculturation and 15 represents the maximum level of acculturation. In addition, the participants reported a mean total score of $11.02(S D=$ 2.72) on the Ethnic/Social Relations subscale of the SASH (4 items) out of a possible score of 20, where 1 represents minimum acculturation and 20 represents a maximum level of acculturation. An average score of 2.99 on each individual item was suggested by the authors as the cut-off to differentiate low versus high acculturated respondents. On average, participants in the current study reported an average score of 2.88 on the Language subscale items, 4.12 on the Media subscale items, and 2.75 on the Ethnic/Social Relations subscale items. While an average score of 4.12 on the Media subscale items indicated high levels of acculturation, the average scores in this sample of 
2.88 and 2.75 on the Language and the Social Relations subscale items, respectively, indicated more moderate levels of acculturation.

Table 3 summarizes participants' reports of acculturative stress ratings measured by the ASSIS (Sandhu \& Asrabadi, 1994). Participants reported a mean total score of $20.12(S D=6.47)$ on the Perceived Discrimination subscale of the ASSIS (8 items), out of a possible score of 40 , where 1 represents a minimum perceived discrimination but a score of 40 stands for the maximum level of perceived discrimination. This sample of international students reported a mean total score of $12.00(S D=3.47)$ on the ASSIS Homesickness subscale (4 items), out of a possible score of 20, where 1 represents a minimum level of homesickness and 20 represents a maximum level of homesickness. The participants reported a mean total score of $12.05(S D=4.16)$ for the Perceived Hate/Rejection subscale of the ASSIS (5 items), out of a possible score of 25, where 1 represents minimum perceived hate/rejection but a score of 25 represents the maximum level of perceived hate/rejection, as well as a mean total score of $7.38(S D=2.75)$ on the Fear subscale of the ASSIS (4 items), of a possible total score of 20, where 1 represents minimum fear, but 20 represents the maximum level of fear. Participants reported a mean total score of $7.30(S D=2.51)$ on the Culture Shock subscale of the ASSIS (3 items), out of a total possible score of 15 , where 1 represents minimum culture shock while 15 represents a maximum level of culture shock, as well as a mean total score of $4.42(S D=$ 1.95 ) on the Guilt subscale of the ASSIS (2 items), of a possible total score of 10, where 1 represents minimum guilt but a score of 10 represents a maximum level of guilt. The students reported a mean total score of $24.55(S D=7.02)$ on the ASSIS Other Concerns subscale (10 items), out of a possible score of 50, where 1 represents a minimum level of 
concerns and 50 represents a maximum level of acculturation-related concerns. Total scores ranged from 36 to 180, and the authors of ASSIS scale have stated that higher scores on this measure indicate that higher levels of acculturative stress are experienced by respondents. While the authors did not mention specific cut-off scores by which a sample can be classified into subgroups based on acculturative stress scores, the results showed evidence that the average ASSIS scores in the sample were generally close to the mid-point of the range of possible values for each of the ASSIS subscales. Hence, the inference can be drawn that the sample reported moderate levels of acculturative stress.

Table 4 summarizes participants' perceptions of peer alcohol use behaviors and injunctive social norms regarding alcohol use. Participating international students reported a total mean score of $8.23(S D=2.55)$ on the Perceived Peer Alcohol Use scale, (DDQ: Collins et al., 1985), out of a possible score of 20. On this measure, scores ranged from 6 , the minimum amount of perceived peer alcohol consumption to a score of 20 , which represents the maximum level of perceived peer alcohol consumption. Two items on this scale measured quantity and frequency of perceived peer alcohol use. While participants reported a mean score of $4.21(S D=1.77)$ on the Quantity item, they reported a mean score of $4.02(S D=1.27)$ on the Frequency item. Participants reported a mean score of $3.19(S D=1.07)$ on the injunctive social norms scale. On this measure, scores ranged from 1 to 5 , where the minimum rating (1) represents an extremely conservative perceived injunctive social norm that drinking is never a good thing to do, while the maximum rating (5) represents an extremely liberal perceived injunctive social norm that a frequently being 'drunk' is okay. The mean scores on the DDQ reported in Table 4 suggested that the perceptions of international students regarding peer alcohol 
use and injunctive social norms concerning alcohol use were neither extremely liberal nor extremely conservative.

International students' self-reported alcohol use behaviors are summarized in Table 5. Participants reported a mean score of $1.32(S D=1.03)$ for an alcohol consumption quantity item, on a 0 to 4 scale, where a score of 0 represents the lowest amount of alcohol use, while 4 represents the maximum quantity of alcohol consumption. In addition, participants reported a mean score of $1.08(S D=.91)$ on an alcohol consumption frequency item, on a 0-4 scale, where a score of 0 represents the lowest frequency of alcohol use while 4 represents the maximum frequency of alcohol consumption. They also reported a mean score of $.50(S D=.74)$ for binge drinking frequency on a 0-4 scale, here a score of 0 represents the lowest frequency for binge drinking while 4 represents the maximum frequency of binge drinking. Intercorrelations between Acculturation Variables and Alcohol Use Variables

The next set of analyses examined intercorrelations among acculturation-related variables and alcohol use-related variables. Table 6 summarizes bivariate correlations among SASH acculturation total scale scores (i.e., for language, media, and ethnic relations acculturation) acculturative stress scores, and alcohol use behaviors. Several SASH-derived acculturation scale scores (e.g., the SASH Total score and scale scores for Media and Ethnic Social Relations) were correlated significantly but in a negative direction with the total score for the ASSIS which measures acculturative stress. This finding suggests that more acculturated international students in the sample reported lower levels of total acculturation stress. 
Table 7 summarizes bivariate correlations among international students' alcohol use variables (e.g., their quantity and frequency of alcohol use, binge drinking frequency, and consequences of drinking) and acculturation scores (i.e., the SASH total score) acculturative stress scores (ASSIS total scores), perceived peer alcohol use, and injunctive social norms. While all of the students' alcohol use-related variables were correlated significantly with Perceived Peer Drinking scores and Injunctive Social Norms scores $(p<.01)$, only the frequency of alcohol use measure was correlated significantly with the acculturation total score and acculturative stress total score $(p<.05)$. This finding suggests that acculturation levels and acculturative stress was not correlated significantly with the alcohol use quantity, binge drinking and alcohol use consequences scores of international students. All of the international students' alcohol use-related variables were correlated positively with both their Injunctive Social Norms scores and their Perceived Peer Drinking scores.

Table 8 summarizes bivariate correlations among international students' acculturative stress variables (e.g., Perceived Discrimination, Homesickness, Perceived Hate/Rejection, Fear, Culture Shock, Guilt and Other Concerns) and SASH acculturation scale scores (i.e., Language, Media, Ethnic Social Relations, and the Total score). All of the students' acculturation stress variables except the Guilt score were correlated significantly with Ethnic Social Relations $(p<.01)$. While Guilt and Perceived Discrimination were not correlated significantly with the SASH Media subscale score, the Fear, Culture Shock, Other Concerns, and Total Acculturative Stress scores were correlated significantly with the SASH Media subscale $(p<.01)$, and the Homesickness and the Perceived Hate/Rejection subscales were correlated with the SASH Media 
subscale at a lower magnitude $(p<.05)$. While the Fear and Other Concerns subscales had significant correlations $(p<.01)$, the Culture Shock subscale had a lower magnitude correlation $(p<.05)$ with the SASH Language Acculturation subscale. These findings suggested that several subscales assessing acculturative stress have significant negative correlations with acculturation subscales while other correlations between ASSIS acculturative stress subscales and SASH acculturation subscales were not statistically significant.

Table 9 summarizes bivariate correlations among international students’ ASSIS acculturative stress subscale scores (e.g., Perceived Discrimination, Homesickness, Perceived Hate/Rejection, Fear, Culture Shock, Guilt and Other Concerns) and selfreported alcohol use variable scores (e.g., quantity and frequency of alcohol use, binge drinking frequency and consequence of drinking). While the Culture Shock subscale of the ASSIS was significantly correlated with Quantity, Frequency and total AUDIT scores $(p<.01)$, it was significantly correlated at a lower magnitude with the Binge Drinking variable $(p<.05)$. While the Fear subscale of the ASSIS was correlated significantly with the Frequency and Total AUDIT score variables $(p<.01)$, it was correlated significantly at a lower magnitude with the Alcohol Use Quantity variable $(p<.05)$. Frequency of alcohol use was significantly correlated $(p<.05)$ with the ASSIS Other Concerns subscale and the ASSIS Total Score. These findings suggest that several indices of acculturative stress were correlated significantly with alcohol use variables while several other correlations were not statistically significant. 


\section{Structural Equation Model (SEM) Analyses}

Structural equation modeling (SEM) was implemented to test the proposed causal model with latent variables (see Figure 1). SEM is an extension of the general linear model which allows the testing of multiple regression equations simultaneously. Model fit was evaluated via AMOS 17.0 software, using sample raw data as input and a Maximum Likelihood (ML) solution. The ML estimation method seeks to maximize the $\log$ likelihood, which reflects how likely it is that the observed values of the dependent variables may be predicted from the observed values of the independent variables. The use of the ML estimation technique assumes that: (a) the sample is very large (asymptotic); (b) the distribution of the observed variables is multivariate normal; and, (c) the scale of observed variables is continuous.

\section{Model Fit Summary}

The Chi-Square fit index tests the null hypothesis that an unconstrained model fits the covariance/correlation matrix, as well as the given model. For a good fitting model, the Chi-Square value is not statistically significant. As the Chi-Square statistic is very sensitive to violations of its assumptions, such as those related to the multivariate normality of data and sample size, it is more prone to Type I statistical errors, i.e., when the null hypothesis is rejected but it is not supposed to be rejected. Therefore, in general practice, it is common to examine a range of model fit indices, rather than making decisions based only on the value of the Chi-Square statistic.

\section{Model Fit Indices}

AMOS provides a large number of alternative indices to assess model fit. Each measure is calculated for three different models: (a) the default model, i.e., the model 
defined by the researcher; (b) the independence model, i.e., the model in which variables are assumed to be uncorrelated with the dependent variables; and, (c) the saturated model, i.e., the model with no constraints. Since no constraints are specified for this class of models, the saturated model always fits any data perfectly. In contrast, because of the inclusion of all possible constraints, the independence model does not always fit the data perfectly. These three models represent points on a continuum, with the independence model at one extreme, the saturated model at the other extreme, and the researcherdefined default model demonstrating measures of fit somewhere between these two ends of the spectrum. The default model is rejected when its fit can be demonstrated not to be significantly better than independence model. Brief descriptions of a few important indices of model fit are provided in the following section, which is followed by the presentation of the analysis of the proposed model.

RMSEA. The RMSEA is the root mean square error of approximation. A value for the RMSEA of .08 or less indicates a close fit of the model with regard to available degrees of freedom. Published research states that a value of about 0.08 or less for the RMSEA indicates a reasonable error of approximation (e.g., Browne \& Cudeck, 1993).

PCLOSE. PCLOSE is a $p$ value that tests the null hypothesis that the population RMSEA is no greater than .05. According to Browne and Cudeck (1993), a RMSEA value of .05 or less indicates a close fit of the model to the data being evaluated.

CFI. CFI is the abbreviation for the Comparative Fit Index, which varies between 0 and 1. Values closer to 1.0 indicate good fit and all values above .90 are acceptable.

GFI. The GFI is the abbreviation for the Goodness of Fit Index. Its values vary from 0 to 1 , but it can also yield theoretically meaningless negative values. A GFI value 
of 1 indicates a perfect fit between a specified model and a set of observed data. By convention, the GFI should be equal to or greater than .90 for a good-fitting model.

AGFI. The AGFI is the Adjusted Goodness Fit Index. The AGFI is a variant of the GFI that takes into account the degrees of freedom available for testing the specified model. The AGFI uses mean squares instead of total sums of squares in the numerator and a denominator of 1-GFI. The AGFI is bounded between 0 and 1 , and this measure also can theoretically yield meaningless negative values. By convention, the GFI should be equal to or greater than .90 for a good-fitting model.

$R M R$. The RMR is the abbreviation for the root mean square residual. It is the square root of the mean, squared amount by which the sample variances and covariances differ from the corresponding estimated variances and covariances, estimated on the assumption that the research-defined model is correct. The smaller the RMR, the better the fit between the specified model and the observed data.

\section{Modification Indices}

To make a decision on whether to reject or to accept a model evaluated in SEM, in addition to the examination of model fit indices, researchers often use model respecification methods. Respecification of a model is done either by adding paths to, or eliminating paths from, an existing model. The modification indices (MIs) capture evidence of poor fit, and this information helps the investigator to understand how to respecify the hypothesized model by either adding or eliminating paths between variables to improve the model fit.

Modification indexes (MIs) can be conceptualized as Chi-Square statistic values with one degree of freedom. Specifically, for each fixed parameter specified, the MI 
represents the expected drop in an overall Chi-Square value if the parameter were to be estimated freely in a subsequent run. All freely estimated parameters automatically have MI values equal to zero. Although this decrease in Chi-Square is expected to approximate the MI value, the actual differential can be larger. Associated with each MI is an expected parameter change value which is reported in tables in this dissertation in the column labeled Par Change. The MI statistic represents the predicted estimated change, in either a positive or negative direction, for each fixed parameter in the model and yields important information regarding the sensitivity of the evaluation of fit. Respecification of a Model and Its Exploratory Nature

It is important to recognize that once it is determined that the hypothesized model represents a poor fit to the observed data and a post-hoc process of model respecification is adopted, analyses are no longer being conducted in a confirmatory mode. All model respecification and estimates thereafter are most appropriately considered exploratory. The decision of whether to pursue a particular specification search is best guided by two considerations: (a) whether the estimation of the targeted parameter is substantively meaningful and, (b) whether the respecified model would lead to an over-fitted model. When there is not a substantive rationale to free up the parameter exhibiting the largest MI, then the parameter having the next largest MI value should be considered (Joreskog \& Sörbom, 1993). When the decision is made to adopt a respecification strategy, in addition to examining whether new models demonstrate acceptable values for model fit indices, it is also standard practice to document the degree of improvement in model fit which is measured by a reduction in the Chi-Square statistic. 


\section{Model Evaluation for the Current Study}

To facilitate the presentation and evaluation of the model investigated in the current study, the results are described in the order that they are presented in the AMOS output.

Model summary. Consistent with the path diagram presented in Figure 1, all observed variables operate as dependent variables. All unobserved factors and error terms operate as independent variables in the model.

Identification of the model. As is demonstrated in Figure 1, the model evaluated in the current study had 15 observed variables. Based on the formula, $p(p+1) / 2$, there were 120 data points of information available. Since 34 parameters needed to be estimated, the model was an over-identified model with 86 degrees of freedom (i.e., 12034). At a conceptual level, an over-identified model is one in which the number of estimable parameters is less than the number of data points or degrees of freedom available, which include the variances and covariances of the observed variables. Models meeting this over-identified condition have positive degrees of freedom, which enables researchers to evaluate and to reject null hypotheses if appropriate. Hence, it is crucial to have an over-identified model when conducting SEM analyses.

Recursive model. In this section of the output, the model was listed by AMOS as being recursive. A recursive model is one in which it is not possible to start with any variable in the path diagram of the model and by following a path of single-headed arrows, return to the same variable. The implication of these relations among variables means that no variable in the model has an effect on itself. 
Assessment of Normality. There are two critical assumptions in SEM that require that data employed in these analyses to: (a) be measured on a continuous scale, and (b) have multivariate normal distributions (Byrne, 2001). Byrne (2001) has documented that when distributions of data are increasingly non-normal, Chi-Square values are inflated considerably and standard errors are underestimated. This might, in turn, result in statistically significant estimated indices, that are not in fact statistically significant. The multivariate normality of data used to evaluate the current model was assessed by procedures in AMOS based on the Mardia's test. Table 10 summarizes information related to the assessment of normality drawn from the AMOS output. The first row of Table 10 shows that the lowest score value for the variable ASSIS Other Concerns was 10 and the highest value was 42 . The variable Other Concerns had a sample skewness of .148. The critical ratio, .908 , is the sample skewness divided by its standard error and it had a sample kurtosis of -.368 and critical ratio of -1.129 . The multivariate kurtosis value of 10.646 is Mardia's coefficient. Any kurtosis or skewness coefficient for an individual variable beyond an absolute value of 1.96 indicates significant non-normality. Therefore, in Table 10, there is no evidence that indicates significant non-normality for any particular variable in the model.

While no evidence of non-normality, based on either kurtosis or skewness, emerged from Table 10, Mahalanobis d-squared values were evaluated to identify individual data observations deviating farthest from the center of their distributions. Table 11 lists the top 10 observations in descending order of their Mahalanobis d-squared values. Observation number 213 is the observation farthest from the centroid with a Mahalanobis d-squared value of 37.408; its $\mathrm{p} 1$ value (.000) and $\mathrm{p} 2$ value $(0.221)$ in the 
adjacent columns indicate the probability that the d-squared value for any individual case would exceed the value of 37.408 is $<.000$ and $<.221$. As stated by Byrne (2001), while small numbers in the $\mathrm{p} 1$ column are expectable, the absence of similarly small numbers in the $\mathrm{p} 2$ column indicated that no significant deviations from the central distribution were present. As no significant non-normality indices were identified in this analysis, procedures such as boot-strapping designed to address non-normality were not implemented in the analyses conducted for the current study.

Notes for the model. The next section of the output from the AMOS program informed the investigator that the minimum was achieved, meaning that the estimation process yielded an admissible solution. Next, the AMOS program listed the Chi-Square value (279.1), together with the degrees of freedom (86) and the probability value $(0.00)$ for the statistic.

The null hypothesis postulated that the specification of the model under investigation was valid and the Chi-Square test evaluated the likelihood that this statement was true. The $p$ value associated with the Chi-Square statistic represented the likelihood of obtaining a Chi-Square value based on observed data that exceeds the ChiSquare value when the null hypothesis is true. Thus, higher $p$ values associated with the Chi-Square statistic indicated closer fit between the hypothesized model and the observed data (Bollen, 1989). In the current study, a Chi-Square value of 279.1 with a $p$ value of 0.00 indicated that, given the current data set, the hypothesis summarized in the model represented a poor fit to the observed data.

Further probing into other indices of model fit also reflected the fact that the hypothesized model was not well represented by the sample data. Specifically, the CFI 
value of .855 and RMSEA value of .100 were not indicative of a good model fit. Several other indicators of model fit indices are presented in Table 14. These indicators of model fit also indicated inadequate goodness-of-fit between the hypothesized model and sample data. For example, the values associated with the GFI (.857) and the CFI (.855) indicated poor fit of the model to the observed data. Thus, it was apparent that some modifications of the specifications of the model were needed in order to identify a model that better represented the sample data. These indicators of model misspecification were found via examination of the modification indices (MIs).

\section{Modification Indices}

The MIs and the accompanying expected parameter change statistics related to the initial hypothesized model are presented in Table 12 and Table 13. While suggested regression paths among the observed variables are presented in Table 12, suggested covariances paths between error terms and factors are presented in Table 13. Only the top 15 parameters in the descending order of MIs are presented in each table. An initial focus on regression paths, based on their MI values and parameter change values, highlights the one with the highest value ( $\mathrm{MI}=31.346$ with a par change coefficient of 0.571$)$. These values referred to the regression of Guilt on Homesickness. This parameter represented the cross-loading of one factor of the latent variable, Acculturative Stress, onto another factor of that same latent variable. It did not account for any misspecification of the hypothesized model and cannot be fixed with respecification of the model to improve the model fit.

Turning to the next large MIs and pertinent expected change statistics, there was very clear evidence of misspecification associated with the pairing of the Perceived Peer 
Alcohol Use latent variable and the Injunctive Social Norms latent variable (MI = 23.919; par change coefficient $=0.777$ ). This finding meant that if the analysis was repeated, using the regression weight for Injunctive Social Norms to predict Perceived Peer Alcohol Use as a free parameter, the discrepancy would fall by at least 23.919 and its estimate would become smaller by approximately 0.777 , compared to the original analysis. The Injunctive Social Norms latent variable measured the participants' perceptions of social norms regarding alcohol use and the Perceived Peer Alcohol Use latent variable measured participants' perceptions of the amount and frequency of alcohol use by their peers. There was a strong rationale for the specification of a direct relation between participants' perceptions of social norms for alcohol use and their perceptions of alcohol use by peers. Since the specification of this direct relation made both statistical and conceptual sense, the model was rerun with an added path from the Injunctive Social Norms latent variable to the Perceived Peer Alcohol Use latent variable.

After the next run, the AMOS program output listed the model Chi-Square value (253.8), together with its degrees of freedom $(n=85)$ and probability value $(0.000)$, still indicating poor fit between the specified model and the observed sample data. While other model fit indices improved significantly from the first model evaluated, they also indicated poor model fit. Further examination of MIs suggested another direction for model respecification. Clear evidence of model misspecification was associated with the pairing of error terms e_HS and e_Gt $(\mathrm{MI}=43.084)$ and error terms e_PD and e_HR (MI $=29.091)$. While there were several other large MI values shown in the AMOS output, these two MIs were substantially larger than the remaining MIs. These MIs represented misspecified error covariances. 
The model was rerun, with the addition of correlations between those two identified paths. After the second rerun, the AMOS output again supported respecification of the model by adding two more correlations, i.e., between error terms e_HS and e_CS, and between error terms e_Fr and e_Gt. Generally speaking, the specification of correlated error terms for the purposes of obtaining better indices of model fit is not an acceptable analytic practice. As with other parameters, such respecification must be supported by a strong substantive and/or empirical rationale (Joreskog \& Sörbom, 1993). However, these measurement error covariances appeared to represent systematic, rather than random, measurement errors in item responses. For example, these large MIs may have been derived from characteristics specific either to the measures used or to the participants in the current study. Specifically, the items may have overlapped in content or represented a small omitted factor. In contrast, respondent characteristics such as bias related to response format or social desirability bias may have contributed to the high MI values (Joreskog \& Aish, 1990).

After the third run of the AMOS model, the final model had a Chi-Square value of $146.8(d f=81 ; p=0.00)$. While the $p$ value associated with the Chi-Square statistic still indicated poor fit, several other fit indices suggested that the hypothesized model was well represented by the sample data. In particular, the CFI value of .951 and the RMSEA value of .060 with a non-significant PCLOSE value were indicative of a good fit between the hypothesized model and the sample data. Several other indices of model fit derived from the final model are presented in Table 15.

Taking into account: (a) the feasibility and statistical significance of all parameter estimates; (b) the substantially good fit of the model, with particular reference to the CFI 
(.951) and RMSEA (.060) values; and, (c) the lack of any substantive evidence of model misfit, it was concluded that any further incorporation of parameters into the model would result in an over-fitted model. Although the majority of fit indexes for this model were improved substantially over those drawn from the initial model, there was still some evidence of misfit in the model. For example, the RMR value of 1.055 was, at best, marginally adequate. However, in the interest of parsimony, and given the relative closeness of these MI values, Model 4 was considered to be the best fitting model. Once a model fits well the sample data, further modifications are no longer justified to obtain incrementally better fit because additional modifications may be simply fitting data to small idiosyncratic characteristics of the sample (MacCallum, Roznowski, \& Necowitz, 1992). An over-fitted model: (a) might be fragile in the sense of representing weak effects that were not likely to be replicable; and, (b) may have led to a significant inflation of standard errors (Wheaton, 1987). In addition, it was difficult to justify additional modifications on conceptual grounds, even if they produced improved model fit.

In assessing the extent to which a re-specified model exhibits improvements in fit, it has become customary to determine if the difference in fit between the original and respecified models is statistically significant. This is accomplished by examining the difference in Chi-Square statistics between the two models. The two models are presumed to be nested. Nested models are hierarchically related to one another because the parameter set of one model is a subset of the other model. Therefore, improvement in model fit can be evaluated statistically, with a significant differential in Chi-Square indicating substantial improvement in model fit. Comparison of the final model $\left(\chi_{(81)}^{2}=146.8\right)$ with 
the initial model $\left(\chi_{(86)}^{2}=279.1\right)$, yielded a difference in $\chi^{2}$ value of 132.3 with $5 d f$ (i.e., 86-81 $d f$ ) which was statistically significant. Therefore, this finding indicated that the final model demonstrated substantial improvement in model fit in relation to the initially defined model.

Final Model Statistics

The overall Chi-Square test of model fit was $\left(\chi_{(81)}^{2}=146.8\right)$ with a $p$ value $=0.00$. The RMSEA was 0.060 with $90 \%$ confidence intervals of $>0.044$ to 0.075 . The $p$ value for the test of close fit was 0.138 . The CFI was 0.951 and the GFI was .922 . The standardized root mean square residual was 1.055 . These indices pointed uniformly towards good model fit. Inspection of the residuals and modification indices revealed no significant points of ill-fit in the model. Figure 2 presents the parameter estimates for the structural coefficients in the final model. Standardized coefficients appear on each path, with unstandardized coefficients in parentheses. The residuals indicate the proportion of unexplained variance in the endogenous variables (i.e., they are error variances in standardized form).

\section{Standardized and Unstandardized Path Coefficients}

Figures 2 and 3 present the parameter estimates for the path coefficients of interest. Figure 2 presents standardized coefficients that appear for each parameter of interest while Figure 3 presents unstandardized coefficients for each parameter of interest. Table 16 presents the $95 \%$ confidence intervals for each of the unstandardized path coefficients for the final model. Table 17 presents the standardized path coefficients for the final model. The model suggested a significant impact of Acculturation on Alcohol Use via Acculturative Stress. In addition, the model documents separate 
significant influences of Perceived Peer Alcohol Use, and Perceived Injunctive Social Norms upon participants' Alcohol use. For every one additional reported unit of Acculturation, the Acculturative Stress was predicted to decrease by 0.39 rating scale units $(p$ value $<.001)$. Acculturation accounted for $12 \%$ of the variance in Acculturative Stress ratings. Similarly, for every one additional reported unit of Acculturative Stress, Alcohol Use was predicted to decrease by 0.03 rating scale units $(p$ value $=.027)$. Acculturative stress accounted for $0.03 \%$ of the variance in alcohol use scores.

For every one additional reported unit of Acculturation, Perceived Peer Alcohol Use was predicted to increase by 0.03 rating scale units $(p=.62)$. This path coefficient was not statistically significant. Therefore, Acculturation did not account for a significant amount of variance in Perceived Peer Alcohol Use. Similarly, for every one additional reported unit of Acculturation, Perceived Injunctive Social Norms was predicted to decrease by 0.01 rating scale units $(p=.82)$. This path coefficient was not statistically significant. Therefore, Acculturation did not account for a significant amount of variance in Perceived Injunctive Social Norms. For every one additional reported unit of Perceived Peer Alcohol Use, the participants' Alcohol Use was predicted to increase by 0.10 rating scale units $(p$ value $<.001)$. Perceived Peer Alcohol Use accounted for $11 \%$ of the variance in the participants' Alcohol Use scores. Similarly, for every one additional reported unit of Perceived Injunctive Social Norms, the participants' Alcohol Use was predicted to increase by 0.13 rating scale units $(p$ value $=.01)$. Perceived Injunctive Social Norms accounted for $11 \%$ of the variance in the participants' Alcohol Use. 


\section{Group Differences}

The current research study hypothesized that social support would moderate the relations specified in the model and, in particular, that higher levels of social support would predict lower levels of acculturative stress and lower levels of alcohol use. AMOS assesses the moderating influences of variables by performing multi-group analyses, in which AMOS graphics uses the same path diagram for all groups but allows different parameter values for different groups. By comparing and contrasting the path coefficients and error variances, it can be determined if the groups differ significantly from each other. To find out if the differences are statistically significant, analyses are repeated by constraining parameters in different groups to be equal and examining the $\chi^{2}$ difference from initial model to the new model along with differences in their degrees of freedom. Since the paths are constrained to be equal, degrees of freedom change accordingly. For the difference in degrees of freedom, if the $\chi^{2}$ difference is statistically significant, one can infer that the groups differ significantly for the specified model. When there are no significant group differences, all groups may have either the same path diagram with the same parameter values or parameter values with statistically nonsignificant differences, meaning that there is no significant moderating influence of the grouping variable.

In a scenario where the grouping variable has a significant moderating influence, groups will differ. They might differ from each other to varying extents. Each group may need either a different path diagram or the groups may have the same path diagram but with different parameter values for different groups. The main purpose of a multi-group analysis is to determine the extent to which the groups differ. AMOS performs a single analysis that estimates parameters and tests hypotheses about multiple groups at once. 
This method provides a test for the significance of any differences found between multiple groups and the simultaneous analysis provides more accurate parameter estimates than would be obtained from several separate single-group analyses.

The initial unconstrained model has the same path diagram for all groups and allows different parameter values to be loaded for different groups. The AMOS program has the ability to generate a nested hierarchy of several models, in addition to this initial unconstrained model. Each of these models can be defined to have constraints at varying levels to impose equality across groups. For the current study, analyses involved the specification of three models. Model 1 generated a model in which measurement weights were constant across groups (i.e., the same for the high and low social support groups). Model 2 generated a model in which measurement weights and structural weights were constant across groups. Model 3 generated a model in which all parameters were constant across groups, including structural covariances, structural residuals, and measurement residuals. In summary, Models 1 through 3 generated a hierarchy of models in which each succeeding model contained all of the constraints of the previous models. In the first model, the factor loadings that loaded onto each latent variable were held constant across groups. Then, in the second model, along with the factor loadings, path coefficients between latent variables were held constant. Finally, in the third model, every single parameter in the model was constrained to be equal across the high and low social support groups.

Comparing and contrasting these three models along with the initial unconstrained model helps to determine the extent to which the groups differed significantly. If the $\chi^{2}$ difference for the difference in the degrees of freedom is statistically significant, then the 
group differences are considered to be significant and the grouping variable is documented to moderate the strength of paths in the model at that particular level.

The sample was initially dichotomized into two groups based on the scores for Social Support. Individual observations where the Social Support score was greater than the sample mean score were included in the High Social Support Group and the rest of the participants were classified as members of the Low Social Support Group. The High Social Support Group included 115 observations, compared to the Low Social Support Group which contained the remaining 111 observations. The impact of unequal group size on the results obtained from a multiple group SEM analysis has not been investigated thoroughly in past research, except that it was found that larger groups exert more influence on the results than do smaller groups. Depending on whether inequalities between groups represent the proportions of group membership in the population or not, the generalizability of sample statistics may vary. However, as the difference between the sizes of the groups in this analysis is minimal, any potential bias can be considered to be insignificant.

Table 18 lists the Chi-Square statistic along with the degrees of freedom for all four multiple group comparison models, including the unconstrained model, and Table 19 lists the Chi-Square difference for each model compared to the unconstrained model. Table 20 lists Chi-Square differences that were significant for all of the models, assuming that the unconstrained model was correct. In Table 20, the first row indicates that for 10 degrees of freedom, a $\chi^{2}$ difference of 22.20 is significant $(p<.05)$. This means that constraining measurement weights to be equal across groups differed significantly from the unconstrained model and that the decrease in the Chi-Square statistic was significant. 
Hence, it was concluded that the high and low social support groups differed from each other significantly at the level of the measurement weights that loaded onto each latent factor's variables. For example, the Acculturation Media factor loaded onto Acculturation with a 0.58 path coefficient for the High Social Support Group but with a 0.39 coefficient for the Low Social Support Group. Similar comparisons are listed in Table 20.

Model 2 (structural weights) listed in Table 19 also showed a significant $39.78 \chi^{2}$ difference for the change in degrees of freedom (18). This means that the constraining of regression paths among latent variables to be equal across groups increased the ChiSquare value significantly, confirming that the high and low social support groups differed from each other significantly in terms of structural weights. The models had paths that connected latent variables with significantly different path coefficients. For example, the path that connects Acculturation with Acculturative Stress had a path coefficient value of -0.56 for the High Social Support Group compared to -0.16 for the Low Social Support Group, meaning that individuals from the Low Social Support Group incurred more acculturative stress compared to their counterparts in the High Social Support Group. Social support moderated the relation between Acculturation level and Acculturation Stress. In a similar vein, regression paths that connected (a) Acculturative Stress with participant Alcohol Use, (b) Acculturation with Perceived Injunctive Social Norms also exhibited comparable discrepancies between high and low social support groups with regard to the strength of regression path coefficients. Similar comparisons are listed in Table 21 and Table 22. On contrary, the regression path that connected Acculturation with Perceived Peer Alcohol Use has a path coefficient value of 0.09 for 
both high and low social support groups, indicating nor group difference for this specific relation.

Model 3 (with measurement residuals) which had all of its parameters constrained to be equal across groups showed a significant increment to its Chi-Square value of 70.23 for a difference of 39 degrees of freedom. Based on the above listed statistics, it was concluded that social support has a significant moderating influence and buffers relations between Acculturation and Acculturative Stress, as well as between Acculturation Stress and Alcohol Use.

Additional Data Analysis Issues

The following text will describe several important background issues related to the SEM analysis presented above that need to be mentioned prior to a discussion of the results.

Missing data. Participants in the current study were required to respond to every single item on the questionnaire related to the variables that were assessed. The investigator made it mandatory for the participants who were answering the on-line survey to answer all of the items before they could submit their responses. Hence, for all of the observations in the data set, the data were complete and there were no missing data. This strategy was used by the investigator so as to avoid dealing with non-random missing data.

Excluded variables. While all variables included in analyses were measured by the on-line questionnaire, a few were omitted from analyses due to specific reasons associated with each of those variables. The following text describes each of those reasons. First, several items were developed by the investigator and added to the SASH 
scale, in addition to the standard set of SASH items. Unfortunately, they did not tap exactly what was expected by the investigator. Instead, they demonstrated random item responses and were not correlated with the rest of the items on the SASH scale. Second, two variables were developed by the investigator to add to the Injunctive Social Norms Scale. These items also did not load on the latent constructs that have been documented for this measure and were, therefore, excluded from further analyses.

Finally, six items from the AUDIT scale, which measures negative consequences related to alcohol use behaviors were omitted from SEM analyses, conducted in AMOS. The six AUDIT items were designed to measure clinically significant alcohol dependence symptoms. Since the sample recruited for the current study was limited to international college and graduate students, who are generally psychologically well adjusted and high functioning in comparison to samples recruited from clinical settings, these items did not assess any normally-distributed alcohol use behavior variables, relevant to the sample in the current study. In addition, the goals of the current study were limited to identifying problem alcohol use behaviors including: High frequency of alcohol use and high quantity alcohol use behaviors such as binge drinking, which are relatively common among some subgroups of university students. However, the goals of the current study did not include the modeling of clinically significant alcohol use problems such as alcohol dependence symptoms, which are less common among university students but normative in treatment samples of youth and young adults. Hence, these AUDIT items were excluded from SEM analyses following preliminary examinations of their distributional characteristics and correlations with other constructs in the models evaluated. 


\section{CHAPTER 5: DISCUSSION}

The results of the current study documented significant relations among international students' self-reported scores on measures of acculturation, acculturative stress, perceived alcohol use by peers, injunctive social norms for alcohol use, social support, and alcohol use behaviors. Prior to conducting structural equation modeling (SEM) analyses, descriptive analyses documented statistically significant intercorrelations among measures of: (a) acculturation and acculturation stress; (b) acculturation stress and alcohol use; and, (c) perceived peer alcohol use or injunctive social norms and international students' alcohol use. Evaluation of SEM analyses resulted in a final model that identified: (a) a significant negative path between acculturation level and acculturation stress; (b) a significant negative path between acculturation stress and student alcohol use; (c) a significant positive path between perceived peer alcohol use and international students' alcohol use; and, (d) a significant positive path between injunctive social norms and international students' alcohol use.

Subsequent SEM analyses conducting a multi-group solution confirmed the significant moderating influence of social support for several paths in the final SEM model. Specifically, the path that connected Acculturation with Acculturative Stress had a significantly higher negative path coefficient value for the High Social Support Group compared to the Low Social Support Group, meaning that individuals from the Low Social Support Group reported significantly more acculturative stress compared to their counterparts in the High Social Support Group. In a similar vein, regression paths that connected (a) Acculturative Stress with participant Alcohol Use, and (b) Acculturation with Perceived Injunctive Social Norms also exhibited comparable significant 
discrepancies between the High and Low Social Support groups with regard to the strength of regression path coefficients.

Therefore, the hypothesis that relations between acculturation and international students' alcohol use would be mediated via acculturative stress was partially supported by the results of the final SEM model. In addition, statistically significant direct relations were documented between perceived peer alcohol use or injunctive social norms and international students' alcohol use. Multi-group SEM analyses documented the significant moderating influence of social support on relations between these constructs. In contrast, gender did not moderate significantly these relations. Integration of the Findings of the Current Study with Past Research

Relations between acculturation and acculturative stress. The results of the current study suggested that the association between high levels of acculturation and low levels of acculturative stress can be explained in part by Berry's (1997) Four-Group Model of Acculturation. By describing the process of acculturation within a stress and coping framework, Berry successfully integrated concepts from models of both stresscoping processes and acculturation. He classified the outcomes of processes of acculturation into four distinct categories, i.e., integration, assimilation, separation, and marginalization, based on how individuals attempted to cope with acculturation-related stresses. While individuals who adopted avoidance coping strategies to address acculturative stress were more likely to be classified as manifesting either separation or marginalization as outcomes, individuals who adopted problem-oriented coping strategies were more often classified as manifesting integration or assimilation as outcomes. Integration occurs when acculturating individuals adapt to the host culture while retaining 
elements of their traditional cultures, whereas assimilation results when adaptation to a host culture occurs without the retention of elements of one's traditional culture.

Drawing from Berry's (1997) model, the negative path coefficient between acculturation and acculturative stress documented in the current study may indicate the adoption of problem-oriented coping strategies during acculturation processes negotiated by international students attending FIU. The presence of lower levels of acculturative stress among students reporting higher levels of acculturation, suggests that international students in current sample who vary systematically by acculturation level may vary with regard other factors such as the coping strategies they use to address school-related demands. For example, international students with higher levels of acculturation may be able to access more coping resources and other supports and withstand more adequately stressful experiences related to adaptation to post-secondary education in a host culture and in broader processes of adjustment (Kosic, Mannetti, \& Sam, 2005; Liem, Lim, \& Liem, 2000).

International students are likely to represent a group of immigrants who are more educated and knowledgeable of their new surroundings compared to the entire population of immigrants. As such, they may cope more effectively with the demands of acculturation processes by adopting forms of problem-oriented coping strategies. In addition, as international students become increasingly acculturated, they may learn more effective and efficient coping strategies to address routine daily problems associated with acculturation, thereby incurring lower aggregate levels of acculturative stress. Maladaptive outcomes, such as separation and marginalization, may result more often when individuals do not have access to sufficient individual or social resources to cope 
effectively with specific stressors experienced during ongoing adaptation processes. Individuals either may make inadequate attempts to cope with stressors encountered, or they may adopt avoidance coping strategies. In the current study, results related to the moderating influence of social support showed evidence of more maladaptive outcomes among international students with low social support. Specifically, individuals with lower levels of social support reported higher levels of acculturative stress, suggesting less access to instrumental supports, informational resources, and emotional supports, as well as potentially less use of problem-oriented coping strategies. This finding suggests that international students with lower social support might have had fewer social resources to enhance coping adequately to engage the acculturative processes and thus incurred more acculturative stress, compared to their high social support counterparts.

Relations between acculturative stress and alcohol use outcomes. Results of the current study identified a negative relation between acculturative stress and alcohol use outcomes. While Caetano (1987) and Caetano and Medina Mora (1988) suggested that acculturation modified alcohol use behaviors among immigrants, Chen et al. (1999) and Vega et al. (1993) reported that negative health risk behaviors including alcohol use, increased as acculturation levels increased in general immigrant populations. Several past research studies, such as Balcazar et al. (1996), Gil et al., (2000) and Lovato et al., (1994) have reported positive correlations, while others reported negative correlations between acculturation-related variables and alcohol use behaviors (e.g., Smith, 2000). As mentioned earlier, along with theoretical and methodological variations, sample characteristics including age, gender, ethnicity, and national origin are likely to have contributed to variations in these research study findings. 
These findings provided further evidence for the use of problem-oriented coping strategies by international students in the current sample. In the presence of higher levels of acculturation stress, students in the sample reported lower levels of alcohol use, suggesting that they are not resorting to alcohol use as a strategy to cope with stresses associated with education in a foreign country. Participants' scores on the AUDIT were low on all the items used to measure the consequences of alcohol use. This strongly implies that this sample of international students was largely not involved in problem alcohol use. Few of the participants reported binge drinking or high frequencies of alcohol use.

In addition, social support was found to exert a significant moderating influence on this relation. In general, individuals who reported higher scores for social support also reported lower levels of alcohol use. These results are in line with past research by Mallinckrodt and Leong (1992), and Misra et al. (2003) who found that social supports serve as powerful buffers against stress and help individuals to cope with perceived stress. Therefore, the current study has contributed further evidence for the buffering influence of social support.

Mediating influence of peer modeling and perceived injunctive social norms. While adequate model fit was established, based on several indices of model fit, the potential mediating roles of perceived peer alcohol use and injunctive social norms were not supported in the relation between acculturation level and self-reported alcohol use, based on the lack of statistical significance of the relevant regression paths. Paths connecting acculturation with perceived peer alcohol and perceived social norms did not show statistically significant coefficients, but the coefficients for paths leading directly 
from perceived peer alcohol use and injunctive social norms to participants' alcohol use were statistically significant.

It is possible that among international students, acculturation-related processes may take longer to establish statistically significant relations with constructs such as perceived peer alcohol use and injunctive social norms for the use of alcohol. In the current study, the majority of the sample reported less than three years of residency in the U.S.. Therefore, this may be too short a period of time for them to establish relations with members of the host culture and stable perceptions of peer alcohol use and injunctive social norms regarding alcohol use. While the current study hypothesized that as international students get more and more acculturated, they get more and more exposed to host group American cultural norms related to alcohol use, the length of stay for this sample might be too short to support this proposition.

In addition, as the results of SEM analyses showed the significant moderating influence of social support on the relation between acculturation and acculturative stress, this implies that the participating international students may have established and retained important social relations with friends from their native cultural group, while establishing relations with members of the host country group. The correlational analyses from the current study showed evidence of significant positive correlations between length of stay in the U.S. and acculturation scores. As the length of stay in the U.S. during graduate education is not significantly long in many cases, it may be the case that the majority of international students' peers were from their own cultural group and their perceptions related to peer alcohol use and injunctive social norms were influenced significantly by 
same culture peers rather than by host group peers and host group injunctive social norms.

In line with past research (e.g., Berkowitz \& Perkins, 1986; Chawla, Neighbors, Lewis, Lee, and Larimer, 2007; Neighbors, O'Connor, Lewis, Chawla, Lee, and Fossos, 2008) the current study has provided evidence of the significant influence of perceived injunctive social norms upon alcohol use behaviors among young adults. As predicted, higher levels of perceived injunctive social norms predicted higher levels of international students' alcohol use. This finding means that the more international students perceived alcohol use as an injunctive social norm, the more they tended to report higher scores for alcohol use. Past research has reported that the culture of the United States fosters greater use of alcohol, and that higher levels of acculturation are associated with increased perceptions by youth of these pro-drinking aspects of U.S. culture. However, the current study did not identify a statistically significant path coefficient for the relation between acculturation and perceived injunctive social norms.

In addition, the results of the current study also supported past research findings regarding relations between peers' alcohol use and alcohol use outcomes among young adults. Several past studies, including Donovan, Molina, and Kelly (2009), Velez-Blasini (1997), Christiansen et al. (1982), and Christiansen et al. (1989), found that alcohol expectancies develop through processes of modeling either via interactions in peer groups or exposure to advertisements. However, contrary to expectations and past research findings, paths leading from acculturation level to perceived peer alcohol use and injunctive social norms were statistically insignificant, negating their potential roles as 
mediators of relations between acculturation and alcohol use among international students attending FIU.

Moderating influence of social support. The current study documented that social support had a significant moderating influence upon structural relations in the model tested, highlighting significant group differences in relations between acculturation variables and alcohol use variables. As hypothesized, high social support groups of international students reported lower levels of acculturation stress and lower scores for alcohol use, in contrast to international students who reported lower scores for social support. Past research by Krammer et al. (2004) and Lee et al. (2004) emphasized that international students in general do not receive sufficient social support and that insufficient access to this coping resource might be a significant determinant of resulting acculturative stress. Pederson (1991) and Misra and Castillo (2004) found that with regard to coping with stress, international students were often deprived of their traditional sources of assistance including normative sources of social support, which buffer several types of stresses incurred during acculturation. The findings of the current study added support to past research studies, as high social support was associated with lower reports of acculturative stress, in contrast to the low social support group of international students.

Most non-Western cultures, where the majority of international students at FIU were born and socialized, foster collectivistic social norms and interdependence among members of society in the face of stressors common to cultural group members. Social support serves as a powerful buffer against multiple sources of stress and helps members of these cultural groups to cope effectively with perceived stress (Mallinckrodt \& Leong, 
1992; Misra et al., 2003). Recruitment of social support might include strategies such as communication, ventilation of stress, taking advice, moral support, or financial help. Individuals might seek help from their own social networks, including friends and family members or from the members of the host group. International students might seek social support in various forms from a range of resources, such as moral support from other members of respective student organizations, financial support from financial aid programs offered by universities, or counseling and advising supports from international student services departments. Mechanisms such as these help individuals to express and ventilate their stress, as well as to understand strategies to deal with acculturative stressors.

Gender differences. While the results of the current study showed evidence of the moderating influence of gender on relations in the structural model evaluated, the influence of gender was not statistically significant. The impact of unequal group size on the results obtained in multiple group solutions in SEM analyses has not been investigated extensively in prior research, except for the finding that larger groups exert more influence on the results than do smaller groups. The sample for the current study included 135 female and 91 male international students, which amounts to a significant disparity in group size. The degree to which membership of groups in a sample represent accurately the proportions of those groups in the population influences the generalizability of sample statistics. However, much of past research on international students has not found significant differences in group membership by gender. Assuming the absence of gender difference in some specific populations of international students, the results of the current study may be influenced in part by inappropriate representation 
of the participants by gender and specific results may have limited generalizability. Disproportionate representation of females in the current sample departs from the gender representation of international students at FIU (e.g., 600 men compared to 543 women at the graduate level; 743 men compared to 733 women at the undergraduate level). Use of random sampling and recruitment of a larger sample could limit this potential bias in future studies.

Other potential moderating variables. The potential moderating influence of several additional variables was examined via exploratory analyses, but statistically significant group differences were not identified. These variables included: Ethnicity, length of stay in the U.S., and undergraduate vs. graduate status. The sample used in the current study had non-proportional representation of groups in terms of these variables. For example, the sample included 93 undergraduate students and 133 graduate students. The sample also included 100 students who resided for three or more years in the U.S., compared to 126 students who reported less than three years of residency in the U.S.. In addition, the sample included 64 Asian, 66 Hispanic/Latino, 19 Black, and 42 White students. Unequal group sizes may have contributed to bias in SEM analyses related to the documentation of group differences, potentially casting doubt on their generalizability. Future studies with larger, more representative samples and approximately equal size groups may improve further our current understanding of the potential moderating influences of specific demographic variables.

\section{Implications for Intervention and Prevention}

Student education. International students should be familiarized with adaptive, problem-oriented coping strategies to address the processes related to acculturation and 
associated stressors. For example, educating international students to improve their language skills might facilitate their experience of acculturation processes and make their negotiation more efficient. Parr et al. (1992) and Sadrossadat (1995) found that international students were found to adjust better when they were more proficient at English language usage. Several other past studies also found that international students experienced less stress during transitions to new educational settings when they were proficient at the use of spoken and written English (Sandhu \& Asrabadi, 1994; Yeh \& Inose, 2003).

In addition, educating international students on how to seek out and utilize available university resources, including but not limited to, social support resources in the form of international student organizations, financial aid counseling, or mental health counseling services may help their acculturation processes to be more efficient and less stressful. Studies by Maxwell et al. (2000), Misra et al. (2003), and Constantine et al. (2004) found that during the process of adjustment, the availability of counseling facilities, less formal sources of social support, and financial resources at host universities were significant resources for international students to facilitate their ability to cope with acculturation-related stressors. Fostering the development of international student associations and encouraging international students to join these organizations can provide students with additional social resources to cope effectively with stresses produced during the negotiation of acculturation processes.

International students new to the United States should receive specific attention and information during orientations for new students regarding short- and long-term negative outcomes of problem patterns of alcohol use, and encouraged strongly to 
consider how alcohol and other drug (AOD) use could impact their health and attainment of future goals. Heavy, episodic use of alcohol is associated significantly with a host of negative or risk behaviors ranging from use of other substances, to other health risk behaviors, and sexual risk behaviors. Several empirical studies have documented significant relations between problematic college alcohol use patterns and a broad range of health risk behaviors (Benette, 1999; Turissi, 2000; Wechsler et al., 1994, 1997, 2000; Wolburg, 2001). Letters, pamphlets, flyers, campus newsletters, and other appropriate media should be used to channel tailored health communications to international students to provide critical information regarding these issues and to motivate positive forms of decision-making and health behaviors. In addition, peer mentors can be a significant social resource for disseminating relevant health communications, as well as for promoting positive health behaviors in peer group social contexts.

Peer mentors. Peer mentors could be designated and assigned to each chartered international student organization on campus, and trained to observe and report other students' risk behaviors, such as maladaptive patterns of AOD use or other risk behaviors that occur in social contexts (Bramson, 1999). Designated peer mentors should be trained extensively and empowered to discuss positive and self-protective health behaviors with international student peers. Peer mentors can also be trained to act as role models and health advocates for members of their student association. Campus Student Affairs organizations can be trained to identify student organization members who are opinion leaders, and work with them to identify and implement appropriate social influence strategies to address pertinent health risk behaviors for specific student groups (e.g., the negative consequences of heavy, episodic alcohol use). International student leaders can 
be designated as peer mentors and encouraged help to identify and refer to intervention other students who misuse alcohol and engage in alcohol-related risk behaviors. Past research (Bramson, 1999; Buncher, 1997) has found that peer mentoring is very effective for the goal of reducing alcohol and drug use among college students. Hence, peer mentoring can be assumed to be a promising risk behavior reduction strategy when implemented during acculturation to post-secondary education in a host country.

Monitoring and surveillance. Alcohol use problems are often assumed to be influenced significantly by peers (e.g., because of role modeling or because of their shared environment). Students involved in problem drinking or who encourage peers to get involved with alcohol misuse should be identified when possible and monitored at frequent intervals. Programs to change campus social norms regarding alcohol use and ongoing surveillance of sales outlets is essential to lowering the demand for, and easy access to alcohol by college students. Strict enforcement of anti-alcohol policies in campus areas to discourage alcohol abuse by students, combined with consistent punishment for those who violate advertised campus policies will help to inhibit students' problem drinking (Wechsler, Lee, Nelson, \& Lee, 2003).

\section{Limitations}

There are some important limitations of the current study that merit discussion. These include: the reliability of self-reports by students, the possible cultural biases of the instruments, the inability to manipulate experimental variable to make causal statements, the issue of not having a control group, and limited generalizability.

Reliability of self-report data. Limitations associated with self-report data reduce the validity of self-report data, due to potential bias. For example, students may both 
underreport or exaggerate either their alcohol use behaviors (both the frequency and quantity of alcohol use) or other health risk behaviors. This might be the result of students' attempts to address social desirability, subjective biases while answering questionnaire items, the values they place on the importance of specific research studies, or the perceived importance of data accuracy for research. In addition, restriction of response choices for specific continuous variables might either under-represent or overrepresent students' behavior. According to Smith et al. (1995) the self reports of students regarding alcohol and drug use should be validated by collateral peer reports. Del Boca and Darkes (2003) also emphasized the importance of collateral reports for the validation of youths' self-reported AOD use. Their study raised concerns about whether response accuracy is influenced by and interacts with social desirability and task demand. While many studies that examined reliability and validity of self-report data found self-report data to be reasonably valid and reliable, these authors recommended strongly research that examines processes underlying response behaviors to improve the validity and reliability of self-report data on AOD use.

Inability to draw causal relations and the issue of a control group. While variables such as stresses reported as a consequence of acculturation by international students are often used to try to draw causal relations, the temporal ordering of these variables was not established in the current study. Instead, correlations or global associations were documented. To draw causal relations, along with the establishment of the temporal ordering of variables (i.e., a variable assumed to cause a change in an outcome should occur first) and correlations among variables (which are often assumed to represent cause and effect), other plausible alternative explanations that may show 
causal relations should be ruled out (Pedhazur \& Schmelkin, 1991; Shadish, Cook, \& Campbell, 2002). Longitudinal studies that may help establish the temporal ordering of key risk behaviors or independent and dependent variables are essential and studies that examine and rule out other plausible alternative explanations are needed for the research domain addressed by the current study.

While SEM models are described by past research as able to draw causal relations, lack of a control group in the current study to compare against and the lack of clear temporal ordering of variables may have reduced the strength of conclusions reached in this study. Even though this study used a non-experimental design, the establishment of an appropriate control group to make comparisons against regarding alcohol use was absent. Alternatively, when making inferences regarding causal relations in cross-sectional studies, endogenous variables, also called as instrumental variables, may influence both the causal and outcome variables. This could be measured by considering those instrumental variables while evaluating model fit.

Limited generalizability. Several international student associations promoted the study and sent their members reminders in accordance with researcher's request. Students who were not affiliated with any international student association or other organization were likely to have received fewer reminders and may have had lower motivation to participate in the study. This might have impacted the representation of several subgroups of international students at Florida International University in the final sample. In addition, international students' familiarity with research participation, and in particular, participation in web-based studies might also have impacted their willingness to volunteer, and ultimately, their representation in the final sample. While several 
international student associations represented international students from their respective countries, some international students might not have been represented by any student association. This may be a limitation for the generalizability of the results of the current study.

\section{Cross Cultural Validity}

Imposing culturally invalid concepts. Ethnic minorities integrate gradually into mainstream American culture during acculturation processes. During this phase of assimilation or integration processes, imposing mainstream or privileged group values upon acculturating individuals is not appropriate, including applying mainstream theories and the usage of mainstream scales which may make little sense. The assumption that dominant group values and norms are rational and normative contributes to ignorance of, and the overlooking of the values and norms of minority groups. For example, concepts like binge drinking might be relevant and meaningful in United States bar culture but far less meaningful elsewhere. Hence, questionnaire items that inquire whether participants have consumed 5 or more drinks in a sitting may not mean much to respondents outside the United States. Similarly, items related to social relations in acculturation questionnaires might hold different meanings for individuals who come from collectivistic cultures compared to those who come from individualistic cultures.

Another example could be the gender sensitivity of assessments. In general, women need lower quantities of alcohol, compared to men, to reach equal blood alcohol levels (Wilsnack \& Wilsnack, 2002). This can be attributed to women's lower metabolism rates compared to those of men. Studies by Wechsler (1994) also have emphasized the importance of defining "binge drinking" differently for men and women 
(i.e., 5 drinks for men, compared to 4 drinks for women). However, the National College Health Association (NCHA) protocol does not contain a differential criterion for binge drinking for men and women. In the current study, binge drinking was defined as 5 or more drinks irrespective of gender, and this variable might have inappropriately assessed women's alcohol use patterns and the conclusions drawn from this study might be vulnerable to criticism regarding their generalizability.

Neglect of culture and cultural insensitivity. Certain traits that are not developed in the dominant culture might be overlooked and ignored, which may be very important for that minority group and may influence many other things for that group. Certain contextual influences might initiate and promote development of a particular behavior. If those contextual influences are not present for the mainstream dominant group, they may be ignored even while doing research on the minorities who have the privilege of those specific contexts. This promotes an undue insensitivity towards minority cultures. They suppress, bias, defect the cultural meanings (Rogler, 1999).

Use of Global Measures in the Current Study. The Social Support Questionnaire (SSQ) measured the construct of social support at a very generic and global level. While this global measurement instrument assessed several aspects of social support, it did not include items that were specific to international students' potentially different support systems and the unique environments of international students in which they recruit social support. Similarly, the measures of alcohol use included in the current study were non-specific, i.e., not specific to any particular culture. This was a deliberate methodological decision to use a global scale, one that was not specific to any particular cultural context, as opposed to culture-specific measures. This choice was made because 
the sample used in the current study was diverse and it did not represent a particular culture. Instead, a wide range of cultures were represented in the current study. The global nature of several of the measures in the current study may be a significant limitation that may pose a threat to the internal validity of the current study.

\section{Future directions}

While the current study focused on the moderating influence of social support and also explored the influence of gender on relations between acculturation-related variables and alcohol use variables, it is equally important to examine the influence of ethnicity and other relevant cultural factors on these same relations. To understand fully the interplay between the individual and the host environment, cultural influences also need to be considered (Anderson, 1991). In addition, the development of more culturallysensitive assessment tools, with increased respect for culture-specific issues, will be of tremendous help for future investigations conducted among specific culturally diverse populations. It is necessary in future studies to assess potential factors that might contribute to these significant group differences. Culture-related factors, biological/physiological factors, or the influence of familial or other social environments on international students may be some of the underlying factors that are promising targets for future investigations. Specific cultural practices related to alcohol use might be culturally sensitive and may not develop similarly across, or be applicable to, multiple cultural groups. Research that identifies these culturally-specific factors may help in the design of more effective intervention and prevention programs (Wilsnack \& Wilsnack, 2002). Further examination of culturally-bound behaviors might contribute significantly 
to future research as these studies might identify risk, protective or resilience factors that improve the designs of currently available intervention and prevention programs.

The results of the current study suggested the need for longitudinal studies that more clearly delineate potential causal relations between acculturation-related variables and alcohol use. Future studies may also help to better describe the nature of differences in these relations by potential moderating factors such as length of stay, age, ethnicity and gender. 


\section{REFERENCES}

Adler, P.S. (1975). The transitional experience: An alternative view of cultural shock. Journal of Humanistic Psychology, 15, 13-23.

Allen J. P., Litten, R. Z., Fertig, J. B., \& Babor, T. (1997). A review of research on the Alcohol Use Disorders Identification Test (AUDIT). Alcoholism: Clinical and Experimental Research, 21, 613-619.

American Psychiatric Association. (1994). Diagnostic and Statistical Manual of Mental Disorders (4th ed) (DSM-IV). Washington, DC: APA.

Anderson, L. E. (1994). A new look at an old construct: Cross-cultural adaptation. International Journal of Intercultural Relations, 18, 293-328.

Anderson, L.P. (1991). Acculturative stress: A theory of relevance to black Americans. Clinical Psychology Review, 11, 685-702.

Baer, J. S., Stacy, A., \& Larimer, M. (1991). Biases in the perception of drinking norms among college students. Journal of Studies on Alcohol, 52, 580-586.

Balcazar, H., Peterson, G., \& Cobas, J. A. (1996). Acculturation and health-related risk behaviors among Mexican-American pregnant youth. American Journal of Health Behavior, 20, 425-433.

Barona, A., \& Miller, J. A. (1994). Short Acculturation Scale for Hispanic Youth (SASHY): A preliminary report. Hispanic Journal of Behavioral Sciences, 16, 155-162.

Barrett, M. E., Joe, G. W., \& Simpson, D. D. (1991). Acculturation influences on inhalant use. Hispanic Journal of Behavioral Sciences. Special Issue: Inhalant Use by Mexican American Youth: Findings from a Longitudinal Study 13, 276-296.

Bennette, P., Smith, C., \& Nugent, Z. (1991). Patterns of drinking in Wales. Alcohol and Alcoholism, 26, 367-374.

Ben-Shalom, U., \& Horenczyk, G. (2003). Acculturation orientations: A facet theory perspective on the bidimensional model. Journal of Cross-Cultural Psychology, 34, 176-188.

Ben-Zur, H. (2005). Coping, distress, and life events in a community sample. International Journal of Stress Management, 12, 188-196.

Berkowitz, A. D., \& Perkins, H. W. (1986). Problem drinking among college students: A review of recent research. Journal of American College Health, 35, 21-28. 
Berry, J. W. (1980). Acculturation as varieties of adaptation. In A M. Padilla (Ed.), Acculturation: Theory, models and some new findings (pp. 9-25). Boulder, CO: Westview Press.

Berry, J. W. (1985). Psychological adaptation of foreign students in Canada. In R. Samuda \& A. Wolfgang (Eds.), Intercultural counseling and assessment: Global perspectives (pp. 235-248). Lewiston, NY: Hogrefe.

Berry, J. W. (1997). Immigration, acculturation, and adaptation. Applied Psychology: An International Review, 46, 5-34.

Berry, J. W., \& Annis, R. C. (1974). Acculturative stress: The role of ecology, culture and differentiation. Journal of Cross-Cultural Psychology, 5, 382-406.

Black, J. S., \& Mendenhall, M. (1990). Cross-cultural training effectiveness: A review and a theoretical framework for future research. Academy of Management Review, $15,113-136$.

Black, S. A., \& Markides, K. S. (1993). Acculturation and alcohol consumption in Puerto Rican, Cuban-American, and Mexican-American women in the United States. American Journal of Public Health, 83, 890-893.

Bollen, K. A. (1989). Structural equations with latent variables. Oxford, England: John Wiley \& Sons.

Bramson, J. H. (1999). The impact of peer mentoring on drug avoidance self-efficacy and substance use. (mentoring). Dissertation Abstracts International: Section B: The Sciences and Engineering, 60, 1843.

Bulthuis, J. D. (1986). The foreign students today: A profile. New Directions for Student Services, 36, 19-27.

Buncher, R. M. (1997). The replicability and transferability of a school-based substance abuse prevention program to a community-based setting. Dissertation Abstracts International: Section B: The Sciences and Engineering, 57(10-B), 6559.

Byrne, B. M. (2001). Structural equation modeling with AMOS: Basic concepts, applications, and programming. Mahwah, NJ, US: Lawrence Erlbaum Associates Publishers.

Caetano, R. (1987). Acculturation and drinking patterns among U.S. Hispanics. British Journal of Addiction, 82, 789-799. 
Caetano, R., \& Medina Mora, M. E. (1988). Acculturation and drinking among people of Mexican descent in Mexico and the United States. Journal of Studies on Alcohol, $49,462-471$.

Carey, K. B., Borsari, B., Carey, M. P., \& Maisto, S. A. (2006). Patterns and importance of self-other differences in college drinking norms. Psychology of Addictive Behaviors, 20, 385-393.

Carr, J. L., Koyama, M., \& Thiagarajan, M. (2003). A Women's support group for Asian international students. Journal of American College Health, 52, 131-134.

Catanzaro, S. J., \& Laurent, J. (2004). Perceived family support, negative mood regulation expectancies, coping, and adolescent alcohol use: Evidence of mediation and moderation effects. Addictive Behaviors, 29, 1779 -1797.

Chawla, N., Neighbors, C., Lewis, M. A., Lee, C. M., \& Larimer, M. E. (2007). Attitudes and perceived approval of drinking as mediators of the relationship between the importance of religion and alcohol use. Journal of Studies on Alcohol and Drugs, $68,410-418$.

Chen, X., Unger, J. B., Cruz. T. B., \& Johnson, C. A. (1999). Smoking patterns of Asian American youth in California and their relationship with acculturation. Journal of Adolescent Health, 24, 321-328.

Cheng, D., Leong, F. T. L., \& Geist, R. (1993). Cultural differences in psychological distress between Asian and Caucasian American college students. Journal of Multicultural Counseling and Development, 21, 182-190.

Cho, Y. (2003). Suicidal ideation, acculturative stress and perceived social support among Korean adolescents. Dissertation Abstracts International: Section B: The Sciences and Engineering, 63, 3907.

Christiansen, B. A., Goldman, M. S., \& Inn, A. (1982). Development of alcohol-related expectancies in adolescents: Separating pharmacological from social-learning influences. Journal of Consulting and Clinical Psychology, 50, 336-344.

Christiansen, B. A., Smith, G. T., Roehling, P. V., \& Goldman, M. S. (1989). Using alcohol expectancies to predict adolescent drinking behavior after one year. Journal of Consulting and Clinical Psychology, 57, 93-99.

Chung, R. C., Bemak, F., \& Wong, S. (2000). Vietnamese refugees' level of distress, social support and acculturation: Implications for mental health counseling. Journal of Mental Health Counseling, 22, 150-161.

Church, A. T. (1982). Sojourner adjustment. Psychological Bulletin, 91, 540-572. 
Cicchetti, D. (1989). How research on child maltreatment has informed the study of child development: Perspectives from developmental psychopathology. In Cicchetti, Dante (Ed); Carlson, Vicki (Ed). (1989). Child maltreatment: Theory and research on the causes and consequences of child abuse and neglect. (pp. 377431). New York: Cambridge University Press.

Collins, R. L., Parks G. A., \& Marlatt, G. A. (1985) Social determinants of alcohol consumption: The effects of social interaction and model status on the selfadministration of alcohol. Journal of Consulting and Clinical Psychology, 53, 189-200.

Compas, B. E., Malcarne, V. L., \& Fondacaro, K. M. (1988). Coping with stressful events in older children and young adolescents. Journal of Consulting and Clinical Psychology, 56, 405-411.

Constantine, M. G., Okazaki, S., \& Utsey, S. O. (2004). Self-concealment, social selfefficacy, acculturative stress, and depression in African, Asian, and Latin American international college students. American Journal of Orthopsychiatry, $74,230-241$.

Davis, T. M. (Ed.). (2002). Open doors 2001/2002: Report on international education exchange. New York: Institute of International Education.

Del Boca, F., \& Darkes, J. (2003). The validity of self-reports of alcohol consumption: state of the science and challenges for research. Addiction, 98, 1-12.

Donovan, J. E., Molina, B. S. G., \& Kelly, T. M. (2009). Alcohol outcome expectancies as socially shared and socialized beliefs. Psychology of Addictive Behaviors, 23, 248-259.

Emmons, K. M., Wechsler, H., Dowdall, G., \& Abraham, M. (1998). Predictors of smoking among US college students. American Journal of Public Health, 88, 104-107.

Essandoh, P. K. (1995). Counseling issues with African college students. The Counseling Psychologist, 23, 348-360.

Felix-Ortiz, M., Newcomb, M. D., \& Myers, H. (1994). A multidimensional measure of cultural identity for Latino and Latina adolescents. Hispanic Journal of Behavioral Sciences, 16, 99-115.

Felner, R. D., Rowlinson, R. I., \& Terre, L. (1986). Unraveling the Gordian knot in life change inquiry: A critical examination of crisis, stress and transitional frameworks of prevention. In S. M. Auerbach \& A. L. Stolberg (Eds.). Crisis intervention with children (pp. 39-63). Washington, DC: Hemisphere. 
Flannery, W. P. Reise, S. P., \& Yu, J. (2001). An empirical comparison of acculturation models. Personality and Social Psychology Bulletin, 27, 1035-1045.

Folkman, S., \& Lazarus, R. S. (1991). Coping and emotion. In Monat, A. \& Lazarus, R. S. (Eds.). (1991). Stress and coping: An anthology (3rd ed.) (pp. 207-227). New York: Columbia University Press.

Gil, A. G., Wagner, E. F., \& Vega, W.A. (2000). Acculturation, familism and alcohol use among Latino adolescent males: Longitudinal relations. Journal of Community Psychology, 28, 443-458.

Gilbert, M. J., \& Cervantes, R. C. (1986). Alcohol services for Mexican Americans: A review of utilization patterns, treatment considerations and prevention activities. Hispanic Journal of Behavioral Sciences, 8, 191-223.

Golden, J. S. (1973). Student adjustment abroad. A psychiatrist's view. International Educational and Cultural Exchange, 8, 28-36.

Grey, M. (2000). Coping and diabetes. Diabetes Spectrum, 13, 167-169.

Guilamo-Ramos, V., Jaccard, J., Johansson, M., \& Tunisi, R. (2004). Binge drinking among Latino youth: Role of acculturation-related variables. Psychology of Addictive Behaviors, 18, 135-142.

Handelsman, M. M., Gottlieb, M. C., \& Knapp, S. (2005). Training ethical psychologists: An acculturation model. Professional Psychology: Research and Practice, 36, 59-65.

Hayes, R. L., \& Lin, H. (1994). Coming to America: Developing social support systems for international students. Journal of Multicultural Counseling and Development, $22,7-16$.

Hechanova-Alampay, R., Beehr, T. A., Christiansen, N. D., \& Van Horn, R. K. (2002). Adjustment and strain among domestic and international student sojourners: A longitudinal study. School Psychology International, 23, 458-474.

Hendershot, C. S., MacPherson, L., Myers, M. G., Carr, L. G., \& Wall, T. L. (2005). Psychosocial, cultural and genetic influences on alcohol use in Asian American youth. Journal of Studies on Alcohol, 66, 185-195.

Hines A. M., Snowden L. R., \& Graves K. L. (1998). Acculturation, alcohol consumption and AIDS-related risky sexual behavior among African American women. Women Health, 27, 17-35. 
Hsia, F., \& Spruijt-Metz, D. (2003). The meanings of smoking among Chinese American and Taiwanese American college students. Nicotine \& Tobacco Research, 5, 837849.

Inman, A. G., Ladany, N., Constantine, M. G., \& Morano, C. K. (2001). Development and preliminary validation of the Cultural Values Conflict Scale for South Asian women. Journal of Counseling Psychology, 48, 17-27.

Johnson, F. W., Gruenewald, P. J., Treno, A. J., \& Taff, G. A. (1998). Drinking over the life course within gender and ethnic groups: A hyperparametric analysis. Journal of Studies on Alcohol, 59, 568-580.

Jones, A.C. (1985). Psychological functioning in black Americans: A conceptual guide for use in psychotherapy. Psychotherapy, Research, Practice and Training, 23, 363-452.

Jöreskog, K.G., \& Aish, A. (1990). Structural equation models applied. PsycCRITIQUES, 35, 687-688.

Jöreskog, K. G., \& Sörbom, D. (1993). LISREL 8: Structural equation modeling with the SIMPLIS command language. Chicago, IL, US: Scientific Software International; Lawrence Erlbaum Associates, Inc.

Kenkel, M. B., Deleon, P. H., Mantell, E. O., \& Steep, A. E. (2005). A psychologist by any other name: Response to commentary by John L. Arnett. Canadian Psychology, 46, 212-214.

Klonoff, E. A., Landrine, H., \& Ullman, J. B. (1999). Racial discrimination and psychiatric symptoms among Blacks. Cultural Diversity \& Ethnic Minority Psychology, 5, 329-339.

Knight, J. R., Sherritt, L., Harris, S. K., Gates, E. C., \& Chang, G. (2003) Validity of brief alcohol screening tests among adolescents: A comparison of the AUDIT, POSIT, CAGE, and CRAFFT. Alcoholism: Clinical and Experimental Research, $27,67-73$.

Kosic, A., Mannetti, L., \& Sam, D. L. (2005). The role of majority attitudes towards outgroup in the perception of the acculturation strategies of immigrants. International Journal of Intercultural Relations, 29, 273-288.

Krämer, A., Prüfer-Kramer, L., Stock, C., \& Tshiananga, J. T. (2004). Differences in health determinants between international and domestic students at a German university. Journal of American College Health, 53, 127-132. 
Lazarus, R. S. (1985). The psychology of stress and coping. Issues in Mental Health Nursing. Special Issue: Stress and Anxiety, 7, 399-418.

Lazarus, R. S. (1997). Acculturation isn't everything. Applied Psychology: An International Review. 46, 39-43.

Lazarus, R. S., DeLongis, A., Folkman, S., \& Gruen, R. (1985). Stress and adaptational outcomes: The problem of confounded measures. American Psychologist, 40, 770-779.

Lee, J., Koeske, G. F., \& Sales, E. (2004). Social support buffering of acculturative stress: A study of mental health symptoms among Korean international students. International Journal of Intercultural Relations, 28, 399-414.

Leong, F. T. L., \& Chou, E. L. (1996). Counseling international students. In P. B. Pedersen, J. G. Draguns, W. J. Lonner, \& J. T. Trimble (Eds.), Counseling across cultures (4th ed., pp. 210-242). Thousand Oaks, CA: Sage.

Leong, F. T., \& Sedlacek, W. E. (1989). Academic and career needs of international and United States college students. Journal of College Student Development, 30, 106111.

Lewis, M. A., \& Neighbors, C. (2004). Gender-specific misperceptions of college student drinking norms. Psychology of Addictive Behaviors, 18, 334-339.

Li, L. W., Seltzer, M. M., \& Greenberg, J. S. (1999). Change in depressive symptoms among daughter caregivers: An 18-month longitudinal study. Psychology and Aging, 14, 206-219.

Liem, R., Lim, B. A., \& Liem, J. H. (2000). Acculturation and emotion among Asian Americans. Cultural Diversity \& Ethnic Minority Psychology, 6, 13-31.

Lin, J.-C. G., \& Yi, J. K. (1997). Asian international students' adjustment: Issues and program suggestions. College Student Journal, 31, 473-479.

Lovato, C. Y., Litrownik, A. J., Elder, J., Nuñez-Liriano, A. et al (1994). Cigarette and alcohol use among migrant Hispanic adolescents. Family \& Community Health, 16, 18-31.

MacCallum, R. C., Roznowski, M., \& Necowitz, L. B. (1992). Model modifications in covariance structure analysis: The problem of capitalization on chance. Psychological Bulletin, 111, 490-504.

Makimoto, K. (1998). Drinking patterns and drinking problems among Asian-Americans and Pacific Islanders. Alcohol Health \& Research World, 22, 270-275. 
Mallinckrodt, B., \& Leong, F. L. (1992). International graduate students, stress, and social support. Journal of College Student Development, 33, 71-77.

Mallinckrodt, B., Shigeoka, S., \& Suzuki, L. A. (2005). Asian and Pacific island American students' acculturation and etiology beliefs about typical counseling presenting problems. Cultural Diversity \& Ethnic Minority Psychology, 11, 227238.

Marín, G., Sabogal, F., Marín, B. V., Otero-Sabogal, R., \& Pérez-Stable, E. J. (1987). Development of a short acculturation scale for Hispanics. Hispanic Journal of Behavioral Sciences. Special Issue: Acculturation Research, 9, 183-205.

Markus, H. R., \& Kitayama, S. (1991). Culture and the self: Implications for cognition, emotion, and motivation. Psychological Review, 98, 224-253.

Matsumoto, D., Hirayama, S., \& LeRoux, J. A. (2006). Psychological skills related to intercultural adjustment. In Wong, P. T. P. \& Wong, L. C. J. (Eds). (2006). Handbook of multicultural perspectives on stress and coping. International and Cultural Psychology Series. (pp. 387-405). Dallas, TX: Spring Publications.

Maxwell, G., Adam, M., Pooran, J., \& Scott, B. (2000). Cultural diversity learning: Effective learning for South Hospitality Management students. Cross Cultural Management - An International Journal, 7, 3-10.

Michailidis, M. P. (1996). A study of factors that contribute to stress within international students. Dissertation Abstracts International Section A: Humanities and Social Sciences, 57, 2333.

Miles, H., Winstock, A., \& Strang, J. (2001) Identifying young people who drink too much: The clinical utility of the five-item Alcohol Use Disorders Identification Test (AUDIT). Drug and Alcohol Review, 20, 9-18.

Misra, R., \& Castillo, L. G. (2004). Academic stress among college students: Comparison of American and international students. International Journal of Stress Management, 11, 132-148.

Misra, R., Crist, M., \& Burant, C. J. (2003). Relationships among life stress, social support, academic stressors, and reactions to stressors of international students in the United States. International Journal of Stress Management, 10, 137-157.

Misra, R., McKean, M., West, S., \& Russo, T. (2000). Academic stress of college students: Comparison of student and faculty perceptions. College Student Journal, 34, 236-245. 
Moos, R. H., \& Schaefer, J. A. (1993). Coping resources and processes: Current concepts and measures. In Goldberger, L. \& Breznitz, S. (Eds). Handbook of stress:

Theoretical and clinical aspects (2nd ed.). (pp. 234-257). New York: Free Press.

Mori, S. (2000). Addressing the mental health concerns of international students. Journal of Counseling and Development, 78, 137-144.

Mouw, T., \& Xie, Y. (1999). Bilingualism and the academic achievement of first and second generation Asian Americans: Accommodation with or without assimilation? American Sociological Review, 64, 232-252.

Moyerman, D. R., \& Forman, B. D. (1992). Acculturation and adjustment: A metanalytic study. Hispanic Journal of Behavioral Sciences, 14, 163-200.

Neighbors, C., O'Connor, R. M., Lewis, M. A., Chawla, N., Lee, C. M., \& Fossos, N. (2008). The relative impact of injunctive norms on college student drinking: The role of reference group. Psychology of Addictive Behaviors, 22, 576-581.

Nicassio, P. M. (1983). Psychosocial correlates of alienation: Study of a sample of Indochinese refugees. Journal of Cross-Cultural Psychology, 14, 337-351.

Nicassio, P. M. (1985). The psychosocial adjustment of the Southeast Asian refugee: An overview of empirical findings and theoretical models. Journal of Cross Cultural Psychology, 16, 153-173.

Nilsson, J. E., Berkel, L. A., Flores, L. Y., \& Lucas, M. S. (2004). Utilization rate and presenting concerns of international students at a university counseling center: Implications for outreach programming. Journal of College Student Psychotherapy, 19, 49-59.

Nwadiora, E., \& McAdoo, H. (1996). Acculturative stress among Amerasian refugees: Gender and racial differences. Adolescence, 31, 477-486.

O’Mally, P. M, Johnston, L. D., \& Bachman, J. G. (1998). Alcohol use among adolescents. Alcohol Health and Research World, 22, 85-93.

Oei, T. P. S., \& Notowidjojo, F. (1990). Depression and loneliness in overseas students. International Journal of Social Psychiatry, 36, 121-130.

Parr, G., Bradley, L., \& Bingi, R. (1992). Concerns and feelings of international students. Journal of College Student Development, 33, 20-25.

Pearlin, L. I. (1989). The sociological study of stress. Journal of Health and Social Behavior, 30, 241-256. 
Pederson, P. B. (1991). Counseling international students. The Counseling Psychologist, $19,10-58$.

Pedhazur, E. J., \& Schmelkin, L. P. (1991). Measurement, design, and analysis: An integrated approach (student ed.). Hillsdale, NJ: Lawrence Erlbaum Associates, Inc.

Perkins, H. W., \& Berkowitz, A. D. (1986). Perceiving the community norms of alcohol use among students: Some research implications for campus alcohol education programming. International Journal of the Addictions, 21, 961-976.

Porier, G. W., \& Lott, A.J. (1967). Galvanic skin response and prejudice. Journal of Personality and Social Psychology, 5, 253-259.

Poyrazli, S., Arbona, C., Bullington, R., \& Pisecco, S. (2001) Adjustment issues of Turkish college students studying in the United States. College Student Journal, $35,52-62$.

Poyrazli, S., Kavanaugh, P. R., Baker, A., \& Al-Timimi, N. (2004). Social support and demographic correlates of acculturative stress in international students. Journal of College Counseling, 7, 73-82.

Puritt, F. J. (1978). The adaptation of African students to American society. International Journal of Intercultural Relations, 2, 90-118.

Randall, M., Naka, K., Yamamoto, K., Nakamoto, H., Arakaki, H., \& Ogura, C. (1988). Assessment of psychosocial stressors and maladjustment among foreign students of the University of the Ryukyus. Psychiatry and Clinical Neurosciences, 52, 289-298.

Redfield, R., Linton, R. \& Herskovits, M. (1936). Memorandum on the study of acculturation. American Anthropologist, 38, 149-152.

Reifman, A., \& Dunkel-Schetter, C. (1990). Stress, structural social support, and well being in university students. Journal of American College Health, 38, 271-277.

Rodriguez, N., Myers, H. F., Morris, J. K., \& Cardoza, D. (2000). Latino college student adjustment: Does an increased presence offset minority-status and acculturative stresses? Journal of Applied Social Psychology, 30, 1523-1550.

Rogler, L. H. (1999). Methodological sources of cultural insensitivity in mental health research. American Psychologist, 54, 424-433. 
Rumpf, H., Hapke, U., Meyer, C., \& John, U. (2002). Screening for alcohol use disorders and at-risk drinking in the general population: Psychometric performance of three questionnaires. Alcohol and Alcoholism, 37, 261-268.

Sadrossadat, S. J. ( 1995). Psycho-social and cultural adjustment among international students at the University of Wollongong. Dissertation Abstracts International: Section B: The Sciences and Engineering, 56, 2388.

Sakamoto, I. (2006). When family enters the picture: The model of cultural negotiation and gendered experiences of Japanese academic sojourners in the United States. Cultural Diversity \& Ethnic Minority Psychology, 12, 558-577.

Saldana, D. H. (1994). Acculturative stress: Minority status and distress. Hispanic Journal of Behavioral Sciences, 16, 116-128.

Sam, D. L., \& Berry, J. W. (1995). Acculturative stress among young immigrants in Norway. Scandinavian Journal of Psychology, 36, 10-24.

Sameroff, A. J., \& Fiese, B. H. (1990). Transactional regulation and early intervention. In S. J. Meisels \& J. P. Shonkoff (Eds). Handbook of early childhood intervention. (pp. 119-149). New York: Cambridge University Press.

Sandhu, D. S. (1994). An examination of the psychological needs of the international students: Implications of counseling and psychotherapy. International Journal of Advanced Counseling, 17, 229-239.

Sandhu, D. S., \& Asrabadi, B. R. (1994). Development of an acculturate stress scale for international students: Preliminary findings. Psychological Report, 75, 435-448.

Sandhu, D. S., Portes, P. R., \& McPhee, S. A. (1996). Assessing cultural adaptation: Psychometric properties of the Cultural Adaptation Pain Scale. Journal of Multicultural Counseling and Development, 24, 15-25.

Sarason, I. G., Levine, H. M., Basham, R. B., \& Sarason, B. R. (1983). Assessing social support: The Social Support Questionnaire. Journal of Personality and Social Psychology, 44, 127-139.

Sarason, I. G., Sarason, B. R., Shearin, E. N., \& Pierce, G. R. (1987). A brief measure of social support: Practical and theoretical implications. Journal of Social and Personal Relationships, 4, 497-510.

Saunders, J. B., Aasland, O. G., Babor, T. F., de la Fuente, J. R., \& Grant, M. (1993). Development of the Alcohol Use Disorders Identification Test (AUDIT): WHO Collaborative Project on Early Detection of Persons with Harmful Alcohol Consumption-II. Addiction, 88, 791-804. 
Schwarzer, R., \& Schwarzer, C. (1996). A critical survey of coping instruments. In M. Zeidner \& N. S. Endler (Eds). Handbook of coping: Theory, research, applications. (pp. 107-132). Oxford, England: John Wiley \& Sons.

Shadish, W. R., Cook, T. D., \& Campbell, D. T. (2002). Experimental and quasiexperimental designs for generalized causal inference. Boston, MA: Houghton, Mifflin and Company.

Shin, K. R. (1994). Psychosocial predictors of depressive symptoms in Korean-American women in New York City. Women \& Health, 21, 73-82.

Smart, J. F., \& Smart, D. W. (1995). Acculturative stress: The experience of the Hispanic immigrant. Counseling Psychologist, 23, 25-42.

Smith, C. C. M. (2000). Effects of acculturation and contributory factors of acculturative stress on alcohol consumption and related problems among African-American women. Dissertation Abstracts International: Section B: The Sciences and Engineering, 61, 1656.

Smith, G. T., Godman, M. S., Greenbaum, P. E., \& Christiansen, B. A. (1995). Expectancy for social facilitation from drinking: The divergent paths of highexpectancy and low-expectancy adolescents. Journal of Abnormal Psychology, 104, 32-40.

Sodowsky, G. R., \& Lai, E. M. W. (1997). Asian immigrant variables and structural models of cross-cultural distress. In A. Booth, A. C. Crouter, \& N. Landale (Eds.), Immigration and the family: Research and policy on U. S. immigrants (pp. 211234). Mahwah, NJ: Erlbaum.

Sodowsky, G. R., Lai, E. W., \& Plake, B. S. (1991). Moderating effects of sociocultural variables on acculturation attitudes of Hispanics and Asian Americans. Journal of Counseling and Development, 70, 194-204.

Spaccarelli, S. (1994). Stress, appraisal, and coping in child sexual abuse: A theoretical and empirical review. Psychological Bulletin, 116, 340-362.SPSS, Inc. (2008). AMOS 17.0. Chicago, IL: SPSS, Inc.

Sue, S. (1999). Science, ethnicity, and bias: Where have we gone wrong? American Psychologist, 54, 1070-1077.

Sutherland, M. E., \& Harrell, J. P. (1986). Individual differences in psychological responses to fearful, racial noxious, and neutral imagery. Imagination Cognition and Personality, 6, 133-150. 
Thoits, P. A. (1995). Stress, coping, and social support processes: Where are we? What next? Journal of Health and Social Behavior, 35, 53-79.

Thomas, S. P., \& Williams, R. L. (1991). Perceived stress, trait anger, modes of anger expression, and health status of college men and women. Nursing Research, 4, 303-307. In Sue, D. W., \& Sue, D. (1999). Counseling the culturally different: Theory and practice. New York: Wiley.

Turrisi, R., Wiersma, K. A., Hughes, K. K. (2000). Binge-drinking-related consequences in college students: Role of drinking beliefs and mother-teen communications. Psychology of Addictive Behaviors. 14, 342-355.

Unger, J. B., Cruz, T. B., Rohrbach, L. A., Ribisl, K. M., Baezconde-Garbanti, L., Chen, X., Trinidad, D. R., \& Johnson, C. A. (2000). English language use as a risk factor for smoking initiation among Hispanic and Asian American adolescents: Evidence for mediation by tobacco-related beliefs and social norms. Health Psychology, 19, 403-410.

Unger, J. B., Trinidad, D. R., Weiss, J. W., \& Rohrbach, L. A. (2004). Acculturation as a risk factor for smoking among Asian American adolescents: Is the association confounded by nationality? Journal of Ethnicity in Substance Abuse, 3, 65-79.

Utsey, S. O., Ponterotto, J. G. (1996). Development and validation of the Index of Race Related Stress (IRRS). Journal of Counseling Psychology, 43, 490-501.

Vega, W. A. (1992). Theoretical and pragmatic implications of cultural diversity for community research. American Journal of Community Psychology, 20, 375-391.

Vega, W. A., Gil, A. G., \& Zimmerman, R. S. (1993). Patterns of drug use among CubanAmerican, African-American, and White non-Hispanic boys. American Journal of Public Health, 83, 257-259.

Vélez-Blasini, C. J. (1997). A cross-cultural comparison of alcohol expectancies in Puerto Rico and the United States. Psychology of Addictive Behaviors, 11, 124141.

Vidulich, R. N., \& Krevanich, F. W. (1966). Racial attitudes and emotional response to visual representation of the Negro. Journal of Social Psychology, 68, 82-93.

Ward, C., Bochner, S., \& Furnham, A. (2001). The psychology of culture shock (2nd ed.) New York, NY: Routledge.

Wechsler, H., Davenport, A., Dowdall, G. (1994). Health and behavioral consequences of binge drinking in college: A national survey of students at 140 campuses, Journal of American Medical Association, 272, 1672-1677. 
Wechsler, H., Davenport, A., Dowdall, G., Grossman, S. J. (1997). Binge drinking, tobacco, and illicit drug use and involvement in college athletics: A survey of students at 140 campuses, American colleges Journal of American College Health, 45, 195-200.

Wechsler, H., \& Kuo, M. (2000). College students define binge drinking and estimate its prevalence: Results of a national survey. Journal of American College Health, 49, 57-64.

Wechsler, H., Lee, J. E., Nelson, T. F., \& Lee, H. (2003). Drinking and driving among college students: The influence of alcohol-control policies. American Journal of Preventive Medicine, 25, 212-218.

Wetter, D.W., Kenford, S.L., Welsch, S.K., Smith, S.S., Fouladi, R.T., Fiore, M.C., \& Baker, T.B. (2004). Prevalence and predictors of transitions in smoking behavior among college students. Health Psychology, 23, 168-177.

Weyers, P., Ising, M., Reuter, M., \& Janke, W. (2005). Comparing two approaches for the assessment of coping: Part i. psychometric properties and intercorrelations. Journal of Individual Differences, 26, 207-212.

Wheaton, B. (1987). Assessment of fit in overidentified models with latent variables. Sociological Methods and Research, 16, 118-154.

Wilsnack, S. C., \& Wilsnack, R. W. (2002). International gender and alcohol research: Recent findings and future directions. Alcohol Research \& Health, 26, 245-250.

Wilton, L., \& Constantine, M. G. (2003). Length of residence, cultural adjustment difficulties, and psychological distress symptoms in Asian and Latin American international college students. Journal of College Counseling, 6, 177-187.

Windle, M., \& Windle, R. C. (2001). Depressive symptoms and cigarette smoking among middle adolescents: Prospective associations and intrapersonal and interpersonal influences. Journal of Consulting and Clinical Psychology, 69, 215-226.

Winkelman, M. (1994). Cultural shock and adaptation. Journal of Counseling and Development, 73, 121-126.

Wolburg, J. M. (2001). The "risky business" of binge drinking among college students: Using risk models for PSAs and anti-drinking campaigns. Journal of Advertising. 30, 23-39.

Wood, M. D., Read, J. P., Mitchell, R. E., \& Brand, N. H. (2004). Do parents still matter? parent and peer influences on alcohol involvement among recent high school graduates. Psychology of Addictive Behaviors, 18, 19-30. 
Yang, B., \& Clum, G. A. (1994). Measures of life stress and social support specific to an Asian student population. Journal of Psychopathology and Behavioral Assessment, 17, 51-67.

Yeh, C. J., \& Inose, M. (2003). International students' reported English fluency, social support satisfaction, and social connectedness as predictors of acculturative stress. Counselling Psychology Quarterly, 16, 15-28.

Yi, J. K., Giseala, J., \& Kishimoto, Y. (2003). Utilization of counseling services by international students. Journal of Instructional Psychology, 30, 333-342.

Ying, Y. (2005). Variation in acculturative stressors over time: A study of Taiwanese students in the United States. International Journal of Intercultural Relations, 29, $59-71$.

Ying, Y., \& Liese, L. H. (1994). Initial adjustment of Taiwanese students to the United States: The impact of post arrival variables. Journal of Cross-Cultural Psychology, 25, 466-477.

Yo, J. (2006). Encyclopedia of multicultural psychology. Thousand Oaks, CA Sage.

Zagefka, H., \& Brown, R. (2002). The relationship between acculturation strategies, relative fit and intergroup relations: Immigrant-majority relations in Germany. European Journal of Social Psychology, 32, 171-188.

Zaleski, E. H., Levey-Thors, C., \& Schiaffino, K. M. (1999). Coping mechanisms, stress, social support, and health problems in college students. Applied Developmental Science, 2, 127-137.

Zhu, S. H., Sun, J., Billings, S. C., Choi, W. S., \& Malarcher, A. (1999). Predictors of smoking cessation in U.S. adolescents. American Journal of Preventive Medicine, 16, 202-207. 
Table 1: Key Demographic Variables Reported by Florida

International University International Students $(N=226)$

\begin{tabular}{lcc}
\hline Variable & $N$ & $\%$ \\
\hline Gender & & \\
\multicolumn{1}{c}{ Male } & 131 & 58.0 \\
$\quad$ Female & 95 & 42.0
\end{tabular}

Year in School

Undergraduates

$93 \quad 41.2$

Graduates

$133 \quad 58.8$

Ethnicity

$\begin{array}{lcc}\text { White/Caucasian } & 42 & 18.6 \\ \text { African-American } & 19 & 8.4 \\ \text { Hispanic/Latino } & 66 & 29.2 \\ \text { Asian/Pacific Islander } & 64 & 28.3 \\ \text { Other } & 35 & 15.5 \\ \text { th of Stay in the United States } & & \\ \text { Less than 1 Year } & 27 & 11.9 \\ 1 \text { Year } & 57 & 25.2 \\ 2 \text { Years } & 42 & 18.6 \\ 3 \text { Years } & 37 & 16.4 \\ 4 \text { or More Years } & 63 & 27.9\end{array}$




\begin{tabular}{|c|c|c|}
\hline & $N$ & $\%$ \\
\hline \multicolumn{3}{|l|}{ Living Arrangements } \\
\hline Alone & 54 & 23.9 \\
\hline With American Friends & 18 & 8.0 \\
\hline With Friends from Native Group & 50 & 22.1 \\
\hline With Other International Students & 23 & 10.2 \\
\hline With Family/Parents & 81 & 35.8 \\
\hline \multicolumn{3}{|l|}{ Campus Living } \\
\hline Off Campus & 203 & 89.8 \\
\hline On Campus & 23 & 10.2 \\
\hline \multicolumn{3}{|l|}{ Marital Status } \\
\hline Married & 44 & 19.5 \\
\hline Single & 178 & 78.8 \\
\hline Divorced & 4 & 1.8 \\
\hline \multicolumn{3}{|l|}{ Children } \\
\hline None & 211 & 93.4 \\
\hline One or Two & 13 & 5.7 \\
\hline Three or More & 2 & 0.9 \\
\hline \multicolumn{3}{|l|}{ Religious Affiliation } \\
\hline Christian & 100 & 44.2 \\
\hline Hindu & 30 & 13.3 \\
\hline Islamic & 11 & 4.9 \\
\hline
\end{tabular}




\begin{tabular}{lcc}
\hline Jewish & 5 & 2.20 \\
Buddhist & 4 & 1.77 \\
Atheist & 32 & 14.16 \\
Other & 44 & 19.47 \\
Country of origin & & \\
India & 32 & 14.2 \\
Venezuela & 15 & 6.6 \\
Trinidad & 14 & 6.2 \\
China & 12 & 5.3 \\
Jamaica & 12 & 5.3 \\
Colombia & 11 & 4.9 \\
Other Country & 130 & 57.5
\end{tabular}

\begin{tabular}{llll}
\hline Age & Mean & SD & Range \\
& 25.9 & 4.3 & $19-42$ \\
\hline
\end{tabular}


Table 2: Acculturation-Related Behaviors for Florida International University International Student Sample.

\begin{tabular}{lcc}
\hline & Mean & SD \\
\hline Language Dimension of Acculturation & 14.38 & 5.82 \\
Media Dimension of Acculturation & 12.37 & 2.68 \\
Ethnic Social Relations Dimension of Accult. & 11.02 & 2.72
\end{tabular}

Note: $N=226$; Responses were coded as: 1= Only Spanish, 2 = More Spanish than English, 3 = Both Equally, $4=$ More English than Spanish, and $5=$ Only English. The SASH contained the following subscales: Language Use (3 items), Media (3 items), and Ethnic Social Relations (4 items). Columns "Mean" and "SD" represent mean and standard deviation values of total scores on each of the subscales. 
Table 3: Acculturative Stress Ratings for Florida International University International Student sample.

\begin{tabular}{lcc}
\hline & Mean & SD \\
\hline Perceived Discrimination & 20.12 & 6.47 \\
Homesickness & 12.00 & 3.47 \\
Perceived Hate/Rejection & 12.05 & 4.16 \\
Fear & 7.38 & 2.75 \\
Culture Shock & 7.30 & 2.51 \\
Guilt & 4.42 & 1.95 \\
Other Concerns & 24.55 & 7.02 \\
\hline
\end{tabular}

Note: $N=226$; Responses coded were $1=$ strongly disagree, 2 = disagree, $3=$ not sure, 4 $=$ agree, and 5 = strongly agree. The ASSIS contained the following subscales: Perceived Discrimination (8 items), Homesickness (4 items), Perceived Hate (5 items), Fear (4 items), Stress Due to Culture Shock (3 items), Guilt (2 items). Columns "Mean" and "SD" represent mean and standard deviation values of total scores on each of the subscales. 
Table 4: Perceived Peer Alcohol Use Behaviors for the Florida International University International Student Sample.

\section{Mean SD}

Quantity of Perceived Peer Alcohol Consumption $\quad 4.21 \quad 1.77$

Frequency of Perceived Peer Alcohol Consumption $\quad 4.02 \quad 1.27$

Total Perceived Peer Alcohol Consumption $\quad 8.23 \quad 2.55$

$\begin{array}{lll}\text { Perceived Inductive Social Norms } & 3.19 & 1.07\end{array}$

Note: $N=226$. Responses coded were $1=$ less than once a month, $2=$ about once $a$ month, $3=$ once or twice a week, $4=$ three or four times a week, $5=$ once a day for the frequency question; and $1=0$ drinks, $2=1-2$ drinks, $3=3-4$ drinks, $4=5-6$ drinks, $5=$ 7-8 drinks for the quantity item. Total Perceived Peer Alcohol consumption is derived by adding these two. For Injunctive social norms $1=$ drinking is never a good thing to do, 2 = Drinking is alright, but a student should never get "smashed" or drunk, $3=$ Occasionally getting drunk is okay, as long as it does not occasionally interfere with grades or other responsibilities, 4 = Occasionally getting drunk is okay, even if it does occasionally interfere with grades or other responsibilities, $5=$ Frequently getting drunk is okay, if that is what people want to do. Columns "Mean" and "SD" represent mean and standard deviation values of total scores on each of the subscales. 
Table 5: Alcohol Use Behaviors for the Florida International University International Student Sample

\begin{tabular}{lcc}
\hline Variable & Mean & SD \\
\hline Quantity of Alcohol Consumption & 1.32 & 1.03 \\
Frequency of Alcohol Consumption & 1.08 & .91 \\
Binge Drinking Frequency & .50 & .74 \\
Consequences of Alcohol Consumption & .69 & 1.50 \\
\hline
\end{tabular}

Note. $N=226$. Responses coded were $0=$ never, $1=$ monthly or less, $2=2-4$ times $a$ month, 3 = 2-3 times a week, 4 = daily or almost daily for Frequency (1 item), $0=I$ do not drink at all, 1 = 1-2 drinks, 2 = 3-4 drinks, $3=5-9$ drinks, $4=10$ or more drinks for Quantity $(1$ item), $0=$ Never, $1=$ less than monthly, $2=$ monthly, $3=$ weekly, $4=$ daily or almost daily for Binge drinking ( 1 item), and $0=$ Never, $1=$ less than monthly, $2=$ monthly, 3 = weekly, $4=$ daily or almost daily Consequences (6 items). Columns "Mean" and "SD" represent mean and standard deviation values of total score on each of the subscales. 
Table 6: Intercorrelations of Acculturation Variables with other Constructs for the Florida International University International Student Sample.

$\begin{array}{lll}\text { ASSIS } & \text { PPD } & \text { ISN }\end{array}$

SASH Variable

\begin{tabular}{lllll}
\hline Language & -.11 & -.10 & .10 & .05 \\
Media & $-.19 * *$ & -.02 & -.06 & .09 \\
Ethnic Social Relations & $-.30 * *$ & .04 & -.04 & .05 \\
Acculturation Total Score & $-.22 *$ & -.06 & -.03 & .07
\end{tabular}

Note. $N=226 . *=p \leq 0.05 ; * *=p \leq 0.01$. ASSIS $=$ Total score on Acculturative Stress Scale for International Students; PPD = Total score on Perceived Peer Drinking; ISN = Total score of Perceived Injunctive Social Norms; AUDIT = Total score on Alcohol Use Disorders Identification Test. 
Table 7: Intercorrelations of Alcohol Use Behaviors with Other Constructs for the Florida International University International Students Sample.

\section{SASH ASSIS PPD ISN}

Alcohol Use Variables

\begin{tabular}{lcccc}
\hline Frequency & $.16^{* *}$ & $-.14^{*}$ & $.25^{* *}$ & $.24 * *$ \\
Quantity & -.01 & -.07 & $.36^{* *}$ & $.27^{* *}$ \\
Binge Drinking Frequency & .01 & -.07 & $.29 * *$ & $.16^{*}$ \\
Total AUDIT Score & .07 & -.11 & $.34 * *$ & $.26^{* *}$
\end{tabular}

Note. $N=226 ; *=p \leq 0.05 ; * *=p \leq 0.01 . \mathrm{SASH}=$ Total score on Acculturation Scale; ASSIS = Total score on Acculturative Stress Scale for International Students; PPD = Total score on Perceived Peer Drinking; ISN = Total score of Perceived Injunctive Social Norms; AUDIT = Total score on Alcohol Use Disorders Identification Test 
Table 8: Intercorrelations between ASSIS Acculturative Stress Variables and SASH Acculturation Variables for the Florida International University International Students Sample.

\begin{tabular}{llllc}
\hline Variable & Language & Media & Ethic Social Relations & SASH \\
Perceived Discrimination & .07 & -.02 & $-.18^{* *}$ & -.02 \\
Homesickness & -.07 & $-.14^{*}$ & $-.29^{* *}$ & $-.18^{* *}$ \\
Perceived Hate/Rejection & -.06 & $-.16^{*}$ & $-.21^{* *}$ & $-.15^{*}$ \\
Fear & $-.18^{* *}$ & $-.18^{* *}$ & $-.19^{* *}$ & $-.23^{* *}$ \\
Culture Shock & $-.15^{*}$ & $-.22^{* *}$ & $-.29^{* *}$ & $-.25^{* *}$ \\
Guilt & .01 & -.08 & -.10 & -.06 \\
Other Concerns & $-.22^{* *}$ & $-.24 * *$ & $-.32^{* *}$ & $-.31^{* *}$ \\
Total ASSIS & -.10 & $-.18^{* *}$ & $-.30^{* *}$ & $-.21^{* *}$
\end{tabular}

Note. $N=226 . *=p \leq 0.05 ; * *=p \leq 0.01$. ASSIS $=$ Total score on Acculturative Stress Scale for International Students; SASH = Total score on Acculturation Scale 
Table 9: Intercorrelations between Acculturative Stress Variables and Alcohol Use Behaviors for the Florida International University International Student Sample.

\begin{tabular}{|l|l|l|l|l|}
\hline Variable & Quantity & Frequency & Binge & AUDIT \\
\hline Perceived Discrimination & -.02 & -.06 & -.04 & -.05 \\
\hline Homesickness & .02 & -.01 & .04 & .02 \\
\hline Perceived Hate/Rejection & -.06 & -.12 & -.05 & -.09 \\
\hline Fear & $-.16^{*}$ & $-.22^{* *}$ & -.10 & $-.19^{* *}$ \\
\hline Culture Shock & $-.19^{* *}$ & $-.26^{* *}$ & $-.16^{*}$ & $-.23^{* *}$ \\
\hline Guilt & .00 & .00 & -.02 & -.01 \\
\hline Other Concerns & -.06 & $-.14^{*}$ & -.05 & -.10 \\
\hline Total ASSIS & -.07 & $-.14^{*}$ & -.07 & -.11 \\
\hline
\end{tabular}

Note. $N=226 ; *=p \leq 0.05,{ }^{* *}=p \leq 0.01$. ASSIS $=$ Total score on Acculturative Stress Scale for International Students; AUDIT = Total score on Alcohol Use Disorders Identification Test 
Table 10: Assessment of Normality from AMOS output

\begin{tabular}{|l|r|r|r|r|r|r|}
\hline Variable & $\min$ & $\max$ & skew & c.r. & kurtosis & c.r. \\
\hline ASSIS_Other Concerns & 10.000 & 42.000 & .148 & .908 & -.368 & -1.129 \\
\hline Guilt & 2.000 & 10.000 & .748 & 4.589 & .031 & .094 \\
\hline Culture Shock & 3.000 & 13.000 & .311 & 1.909 & -.552 & -1.694 \\
\hline Fear & 4.000 & 16.000 & .797 & 4.891 & .465 & 1.427 \\
\hline Perceived Hate Rejection & 5.000 & 25.000 & .284 & 1.743 & -.412 & -1.264 \\
\hline Homesickness & 4.000 & 20.000 & -.008 & -.052 & -.501 & -1.537 \\
\hline Perceived Discrimination & 8.000 & 40.000 & .236 & 1.447 & -.404 & -1.240 \\
\hline Ethnic Social Relations & 5.000 & 19.000 & .212 & 1.302 & -.338 & -1.038 \\
\hline Media & 3.000 & 15.000 & -.973 & -5.970 & .500 & 1.534 \\
\hline Language & 5.000 & 25.000 & .661 & 4.056 & -.659 & -2.022 \\
\hline Frequency & .000 & 4.000 & .260 & 1.597 & -.867 & -2.659 \\
\hline Binge Drinking & .000 & 3.000 & 1.413 & 8.674 & 1.401 & 4.298 \\
\hline Quantity & .000 & 4.000 & .763 & 4.683 & .382 & 1.172 \\
\hline Injunctive Social Norms & 1.000 & 5.000 & -.070 & -.431 & -.198 & -.606 \\
\hline Perceived Peer Drinking & .000 & 14.000 & -.319 & -1.957 & .376 & 1.154 \\
\hline Multivariate & & & & & 10.646 & 3.543 \\
\hline
\end{tabular}


Table 11: Top Ten Observations in Descending Order of Mahalanobis d-squared Values

\begin{tabular}{|r|r|r|r|}
\hline Observation number & Mahalanobis d-squared & $\mathrm{p} 1$ & $\mathrm{p} 2$ \\
\hline 38 & 37.408 & .001 & .221 \\
\hline 1 & 36.301 & .002 & .051 \\
\hline 180 & 35.205 & .002 & .016 \\
\hline 50 & 34.079 & .003 & .007 \\
\hline 224 & 32.487 & .006 & .009 \\
\hline 11 & 32.010 & .006 & .004 \\
\hline 10 & 30.937 & .009 & .005 \\
\hline 162 & 29.649 & .013 & .011 \\
\hline 79 & 28.977 & .016 & .012 \\
\hline 200 & 28.795 & .017 & .006 \\
\hline
\end{tabular}


Table 12: Modification Indices (Regression Weights) from AMOS Output for the Initially Hypothesized Model

\begin{tabular}{lll}
\hline Path & MI & Par Change \\
\hline Homesickness<---Guilt & 31.346 & 0.571 \\
Guilt<---Homesickness & 29.826 & 0.18 \\
Peer Alcohol<---Injunctive Social Norms & 23.919 & 0.777 \\
Injunctive Social Norms <---Peer Alcohol & 23.876 & 0.137 \\
Inj Social Norm <---Peer Alcohol & 23.075 & 0.132 \\
Inj Social Norm <--- Perceived Peer Drinking & 23.075 & 0.132 \\
Perceived Peer Drinking <--- Injunctive Social Norms & 21.381 & 0.694 \\
Perceived Peer Drinking <--- Inj Social Norm & 21.381 & 0.694 \\
Perceived Discrimination<--- Language & 17.942 & 0.234 \\
Perceived Discrimination <----Acculturation & 13.821 & 0.351 \\
Perceived Hate Rejection<---Perceived Discrimination & 13.799 & 0.114 \\
Perceived Discrimination <---Perceived Hate Rejection & 12.298 & 0.272 \\
Culture Shock<---Homesickness & 11.87 & 0.121 \\
Frequency<---Acculturation & 11.7 & 0.049 \\
Ethnic Social Relations<---Homesickness & 11.617 & -0.162 \\
\hline
\end{tabular}

Table 13: Modification Indices (error Covariances) from AMOS Output for the Initially Hypothesized Model

\begin{tabular}{lll}
\hline Path & MI & Par Change \\
\hline e_HS $<-->$ e_Gt & 43.088 & 2.227 \\
e_PD $<-->$ e_HR & 29.098 & 5.129 \\
d_PPd $<-->$ d_ISN & 23.919 & 0.887 \\
e_SN $<-->$ d_PPd & 23.117 & 0.857 \\
e_PPD $<-->$ d_ISN & 21.381 & 0.793 \\
e_PPd $<-->$ e_SN & 20.591 & 0.764 \\
e_HS $<-->$ e_CS & 17.104 & 1.489 \\
e_PD $<-->$ Acculturation & 13.821 & 5.531 \\
e_CS $<-->$ d_Alc & 13.377 & -0.342 \\
e_PD $<-->$ e_HS & 12.554 & -3.385 \\
e_Fq<-->Acculturation & 11.7 & 0.768 \\
e_Lg<-->e_PD & 10.346 & 4.995 \\
e_SR $<-->$ d_ASSIS & 9.332 & -2.288 \\
e_Fr $<-->$ e_Gt & 7.97 & 0.601 \\
e_PD $<-->$ d_PPd & 7.62 & 2.257 \\
\hline
\end{tabular}


Table 14: Model Fit Indices from AMOS Output, Initial Model

\begin{tabular}{llll}
\hline Modification Fit Index & Default model & Saturated model & Independence model \\
\hline$\chi^{2}$ & 279.1 & & \\
$D f$ & 86 & & \\
$\mathrm{p}$ value & 0.000 & & \\
RMSEA & 0.100 & & 0.238 \\
PCLOSE & 0.000 & 0.000 & 0.000 \\
RMR & 1.168 & 1.000 & 4.778 \\
CFI & 0.855 & 1.000 & 0.000 \\
GFI & 0.857 & & 0.456 \\
AGFI & 0.800 & & 0.379 \\
\hline
\end{tabular}

Table 15: Model Fit Indices from AMOS Output, Final Model (Model 4)

\begin{tabular}{llll}
\hline Modification Fit Index & Default model & Saturated model & Independence model \\
\hline$\chi^{2}$ & 146.8 & & \\
$D f$ & 81 & & \\
$\mathrm{p}$ value & 0.000 & & 0.238 \\
RMSEA & 0.060 & & 0.000 \\
PCLOSE & 0.138 & 0.000 & 4.778 \\
RMR & 1.055 & 1.000 & 0.000 \\
CFI & 0.951 & 1.000 & 0.456 \\
GFI & 0.922 & & 0.379 \\
AGFI & 0.885 & & \\
\hline
\end{tabular}

Table 16: 95\% Confidence Intervals for Unstandardized Path Coefficients for Final Model

\begin{tabular}{lll}
\hline Path & Estimate & $95 \%$ CI \\
\hline Peer Alcohol<---Acculturation & .025 & 0.073 to 0.123 \\
Injunctive Social Norms $<---$ Acculturation & -.005 & -0.0442 to 0.0342 \\
Acculturative Stress $<---$ Acculturation & -.387 & -0.5791 to -0.19492 \\
Injunctive Social Norms $<---$ Peer Alcohol & .137 & -0.0860 to .18796 \\
Alcohol Use $<---$ Peer Alcohol & .104 & -0.06088 to 0.1471 \\
Alcohol Use $<---$ Injunctive Social Norms & .133 & 0.03108 to 0.23492 \\
Alcohol Use $<---$ Acculturative Stress & -.028 & -0.0535 to -0.00252 \\
\hline
\end{tabular}


Table 17: Standardized Path Coefficients for Final Model

\begin{tabular}{ll}
\hline Path & Estimate \\
\hline Peer Alcohol<---Acculturation & -.039 \\
Injunctive Social Norms $<---$ Acculturation & .017 \\
Acculturative Stress<---Acculturation & -.351 \\
Injunctive Social Norms $<---$ Peer Alcohol & .326 \\
Alcohol Use<---Peer Alcohol & .326 \\
Alcohol Use $<---$ Injunctive Social Norms & .334 \\
Alcohol Use $<---$ Acculturative Stress & .180 \\
\hline
\end{tabular}

Table 18: Model Fit Indices Across Models from AMOS Output

\begin{tabular}{lllll}
\hline $\begin{array}{l}\text { Model Fit Index } \\
\text { for Default Model }\end{array}$ & Model 1 & Model 2 & Model 3 & Model 4 \\
\hline$\chi^{2}$ & 279.1 & 253.8 & 178.0 & 146.8 \\
$D f$ & 86 & 85 & 83 & 81 \\
p value & 0.000 & 0.000 & 0.000 & 0.000 \\
RMSEA & 0.100 & 0.094 & 0.071 & 0.060 \\
PCLOSE & 0.000 & 0.000 & 0.009 & 0.138 \\
RMR & 1.168 & 1.164 & 1.072 & 1.055 \\
CFI & 0.855 & 0.873 & 0.929 & 0.951 \\
GFI & 0.857 & 0.870 & 0.909 & 0.922 \\
AGFI & 0.800 & 0.817 & 0.868 & 0.885 \\
\hline
\end{tabular}

Table 19: Group Differences Across Social Support Groups, Chi-Square Values along with Degrees of Freedom

\begin{tabular}{lrrrr}
\hline Model & NPAR & CMIN & DF & P \\
\hline Unconstrained & 78 & 248.095 & 162 & .000 \\
Measurement weights & 68 & 270.297 & 172 & .000 \\
Structural weights & 61 & 287.870 & 179 & .000 \\
Measurement residuals & 39 & 318.321 & 201 & .000 \\
\hline
\end{tabular}

Table 20: Group Differences across Social Support Groups, Chi-Square Difference Values along with Degrees of Freedom

\begin{tabular}{lrr}
\hline Model & CMIN difference & DF \\
\hline Unconstrained & 248.095 & 162 \\
Measurement weights & $22.20(270.297)$ & 10 \\
Structural weights & $39.78(287.870)$ & 18 \\
Measurement residuals & $70.23(318.321)$ & 39 \\
\hline
\end{tabular}


Table 21: Group Differences across Social Support, Measurement Weights Difference Values

\begin{tabular}{|c|c|c|}
\hline Path & High Social support & Low Social Support \\
\hline Media $\leftarrow$ Acculturation & 0.58 & 0.39 \\
\hline Ethnic Social Relations $\leftarrow$ Acculturation & 0.45 & 0.21 \\
\hline Hate Rejection $\leftarrow$ Acculturative Stress & 0.69 & 0.62 \\
\hline Homesickness $\leftarrow$ Acculturative Stress & 0.38 & 0.44 \\
\hline Fear $\leftarrow$ Acculturative Stress & 0.35 & 0.56 \\
\hline Culture Shock $\leftarrow$ Acculturative Stress & 0.37 & 0.40 \\
\hline Guilt $\leftarrow$ Acculturative Stress & 0.16 & 0.25 \\
\hline Other Concerns $\leftarrow$ Acculturative Stress & 1.38 & 1.56 \\
\hline Quantity $\leftarrow$ Alcohol Use & 1.03 & 1.03 \\
\hline Binge Drinking $\leftarrow$ Alcohol Use & 0.91 & 0.56 \\
\hline
\end{tabular}

Table 22: Group Differences across Social Support, Structural Weights Difference Values

\begin{tabular}{|c|c|c|}
\hline Path & High Social support & Low Social Support \\
\hline Acculturative Stress $\leftarrow$ Acculturation & -0.56 & -0.16 \\
\hline Peer Alcohol $\leftarrow$ Acculturation & 0.09 & 0.09 \\
\hline Injunctive Social Norms $\leftarrow$ Acculturation & -0.05 & 0.03 \\
\hline Alcohol Use $\leftarrow$ Acculturative Stress & -0.04 & 0.01 \\
\hline Alcohol Use $\leftarrow$ Peer Alcohol & 0.14 & 0.06 \\
\hline Alcohol Use $\leftarrow$ Injunctive Social Norms & 0.09 & 0.16 \\
\hline Injunctive Social Norms $\leftarrow$ Peer Alcohol & 0.16 & 0.10 \\
\hline
\end{tabular}


Figure 1. Hypothesized model

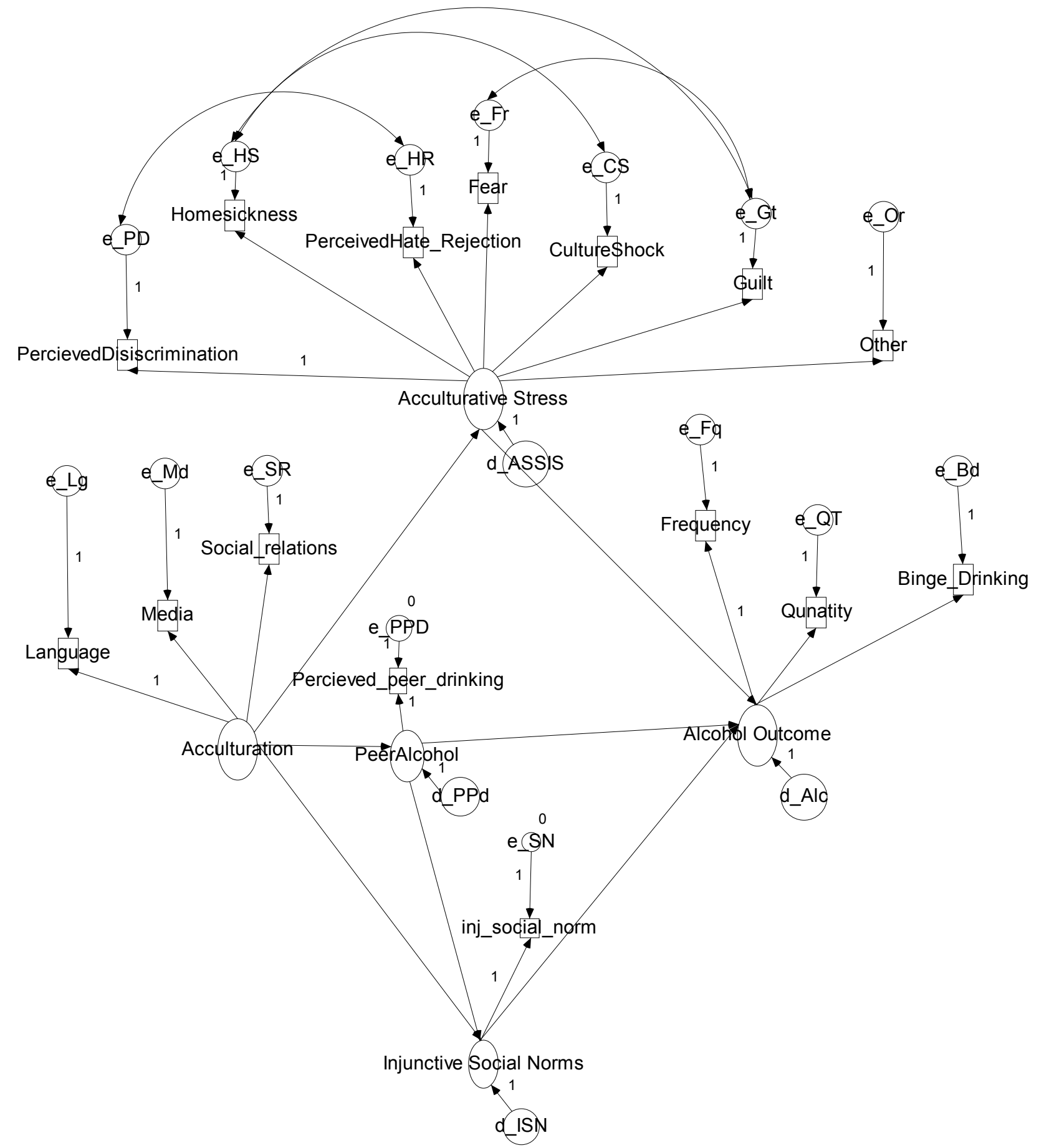


Figure 2. Standardized estimates

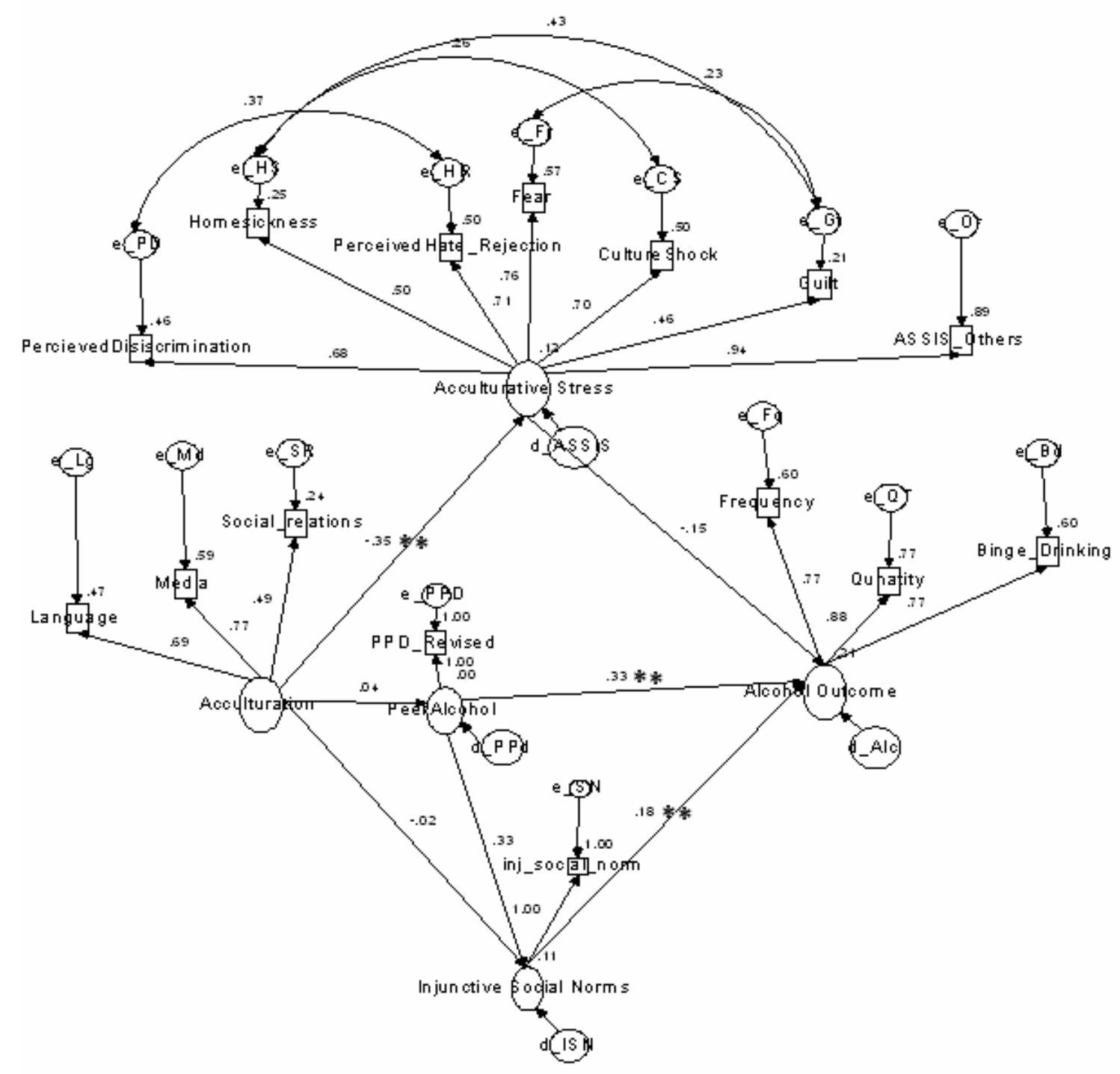


Figure 3. Unstandardized estimates

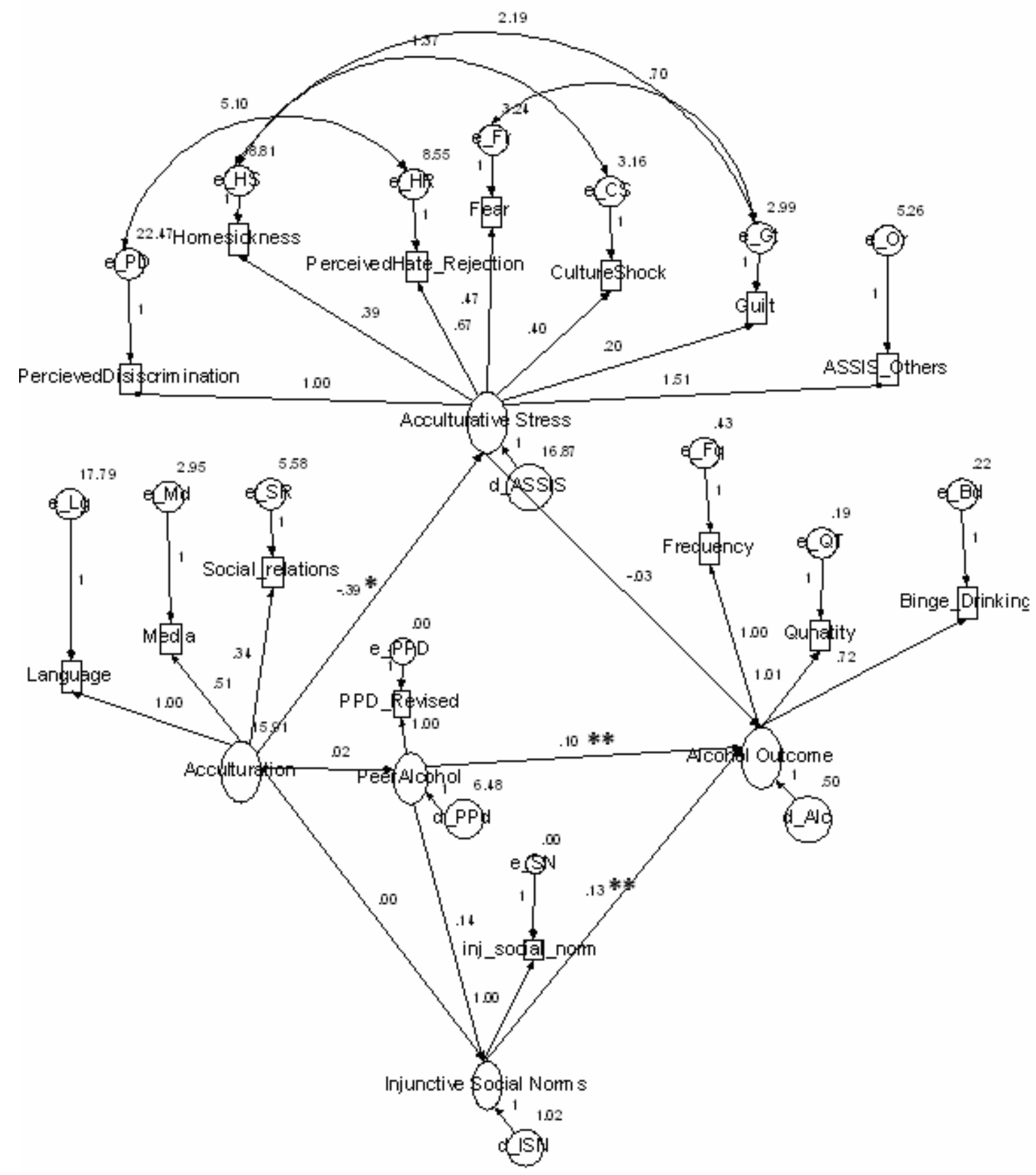


VITA

ANANTH KANAPARTHI

2000

B.H.M.S.

N.T.R Health University

Andhra Pradesh, India

2005

M.S. Psychology

Florida International University

Miami, Florida

2006-2007

Adjunct Instructor

Florida International University

Miami, Florida

2003-2005

Teaching Assistant

Florida International University

Miami, Florida

2002-2003

Research Assistant

Florida International University

Miami, Florida

\section{CONFERENCE PRESENTATIONS}

Oshri, A., Tubman, J. G., Kanaparthi, A. (2006) Psychological maltreatment history among youth in alcohol treatment. Paper presented at the annual international meeting of the Research Society on Alcoholism, Baltimore, MD.

\section{MANUSCRIPTS IN PREPARATION}

Relations between acculturation and acculturative stress in international student populations

Social support as moderator for buffering acculturative stress in international students

Critical evaluation of applying attachment theory and strange situation procedure for deaf-blind infants 